\title{
On-line monitoring of hydrocyclones by use of image analysis
}

\author{
by \\ Magrieta Jeanette Janse van Vuuren \\ Thesis presented in partial fulfillment \\ of the requirements for the Degree \\ MASTER OF SCIENCE IN ENGINEERING \\ (EXTRACTIVE METALLURGICAL ENGINEERING) \\ in the Faculty of Engineering \\ at Stellenbosch University
}

Supervised by

Prof. C Aldrich

March 2011 


\section{DECLARATION}

By submitting this thesis electronically, I declare that the entirety of the work contained therein is my own, original work, that I am the sole author thereof (save to the extent explicitly otherwise stated), that reproduction and publication thereof by Stellenbosch University will not infringe any third party rights and that I have not previously in its entirety or in part submitted it for obtaining any qualification.

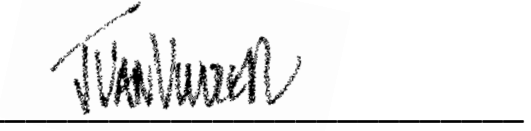

Signature
25 February 2011

Date

Copyright (C) 2011 Stellen6osch University

All rights reserved 


\section{ABSTRACT}

Hydrocyclones are separation devices that are widely used throughout the chemical engineering and mineral processing industries. Although simple in design, the intricate flow structure of the device complicates control. As an alternative to conventional empirical and theoretical modelling, process state monitoring methods have recently been employed as a means to control hydrocyclones. The purpose of process state monitoring methods is to distinguish between the desired operating state with favourable separation, the transition state, and the troublesome operating state of dense flow separation.

In comparison to previously employed monitoring techniques, image analysis of the underflow is regarded as a promising approach. Preliminary studies have indicated that the technique complies with hydrocyclone monitoring requirements: sensitivity, noninvasiveness, sampling times less than one second, robustness and low cost. The primary objective of this study was therefore defined as investigating the feasibility of image analysis of hydrocyclone underflow as a monitoring technique.

Data collection entailed the recording of hydrocyclone underflow for different operating states. Six case studies were performed in total: Gold, Ilmenite, Platreef, Merensky 1, Merensky 2 and Merensky 3 (with the case study names indicating the different ore types used). An image analysis technique, consisting of feature extraction through motion detection, as well as various noise reduction methods, was consequently developed and applied to the video data. Classification of the various operating states was attempted by performing modelling by one-class support vector machines (SVM).

Results indicated that the developed image analysis technique effectively addresses background noise, random noise and system vibration through image enhancement and a motion threshold. Extremely low contrast differences and foreground noise did, however, prove problematic in Ilmenite and Merensky 1 case studies respectively. For the remaining case studies, it was found that the various operating states were identified with high accuracy through one-class SVM classification. This is particularly true for the identification of the troublesome dense flow separation for which extremely low missing alarm rates were obtained ( $0 \%$ in most cases). In terms of practicality, the technique proved to be sensitive, non-intrusive and economical. The sampling time of 30 frames per second and estimated processing to video time ratio of $1: 1$, is furthermore satisfactory. Ultimately, the results indicate that the image analysis of hydrocyclone underflow is a viable monitoring technique.

The robustness of the technique might further be improved by use of backlighting and an air-knife. It is also recommended that future work should focus on testing the monitoring technique on an industrial hydrocyclone setup. 


\section{OPSOMIMING}

Hidrosiklone is skeidingsapparate wat algemeen gebruik word in chemiese ingenieurswese en mineraalprosesserings industrieë. Alhoewel die apparaat ' $n$ eenvoudige ontwerp het, bemoeilik die komplekse interne vloeistruktuur die beheer daarvan. Prosestoestandmoniteringsmetodes is vir hidrosikloonbeheer toegepas as alternatief vir konvensionele empiriese en teoretiese modellering. Die doel van prosestoestandmoniteringsmetodes is om te onderskei tussen die gewenste bedryfstoestand met gunstige skeiding, die oorgangstoestand, en die moeilike bedryfstoestand van digtevloeiskeiding.

In vergelyking met vorige toegepaste moniteringstegnieke, word beeldverwerking van die ondervloei beskou as ' $\mathrm{n}$ belowende tegniek. Voorlopige studies het aangedui dat die tegniek voldoen aan die hidrosikloonmoniteringvereistes: sensitiwiteit, nie-indringendheid, monsternemingstydperke laer as een sekonde, robuustheid en lae koste. Die primêre doelwit van hierdie studie is daarom gedefineer as die ondersoek van die doenlikheid van beeldverwerking van hidrosikloon ondervloei as ' $n$ moniteringstegniek.

Die data versameling het die afneem van hidrosikloon ondervloei vir verskillende bedryfstoestande behels. Ses gevallestudies is in totaal uitgevoer: Goud, Ilmeniet, Platreef, Merensky 1, Merensky 2 en Merensky 3 (die gevallestudie name dui die verskillende erts tipes wat gebruik is aan). ' $\mathrm{n}$ Beeldverwerkingstegniek, wat bestaan uit kenmerkekstraksie deur bewegingsopsporing, asook verskeie geruisverlagingsmetodes, is gevolglik ontwikkel en toegepas op die video data. Klassifikasie van die verskeie bedryfstoestande is beproef deur modellering met enkelklassteunvektormasjiene.

Resultate het aangedui dat die ontwikkelde beeldverwerkingstegniek agtergrond geruis, onreëlmatige geruis en sisteem vibrasie suksesvol aanspreek deur beeldversterking en ' $n$ bewegingslimiet. Beduidende lae kontrasverskille en voorgrond geruis blyk wel problematies in die Ilmeniet en Merensky 1 gevallestudies onderskeidelik. Vir die orige gevallestudies is gevind dat die verskillinde bedryfstoestande met hoë akkuraatheid geïdentifiseer is deur enkelklassteunvektormasjiene klassifisering. Dit is veral waar vir die identifisering van die moeilike digtevloeiskeiding waarvoor beduidende lae vermistealarmmaatstawwe behaal is ( $0 \%$ in die meeste gevalle). Aangaande die praktiese aspekte, blyk die tegniek sensitief, nie-indringend en ekonomies. Die monsternemingstydperk van 30 raampies per sekonde en die beraamde prosesserings- tot videotyd verhouding van $1: 1$, is ook voldoende. Ten slotte dui die resultate daarop dat die beeldverwerking van hidrosikloon ondervloei 'n uitvoerbare moniteringstegniek is. 
Die robuustheid van die tegniek sou verder verbeter kon word deur gebruik te maak van agtergrondverligting en ' $n$ lugspuit. Dit word ook aanbeveel dat toekomstige werk op die toetsing van die moniteringstegniek op ' $n$ industriële hidrosikloon toestel moet fokus. 


\section{ACKNOWLEDGEMIENTS}

The completion of a post-graduate study on a part-time basis might initially be perceived as an overwhelmingly mammoth and exhausting task. Although this is true to a large extent, the load can be lightened significantly by the support and guidance of certain individuals. Throughout the course of my study, various individuals helped to make my own journey an enlightening and joyous one. I would therefore like to express my sincere gratitude towards the following persons for their contributions:

To my supervisor, Professor Aldrich, for your valuable technical guidance and encouragement, but most of all, for teaching me how to 'Eat an elephant... Stukkie vir stukkie'.

To my helping hands, Nikhil and Vincent, for assisting with the physical labour required during experimental work.

To the workshop and secretarial staff, for your assistance with countless technical aspects.

To Lidia, for your advice, being a great sounding board and for a cherished friendship.

To my remarkable parents, Johan and Magrieta, for providing me with excellent opportunities and enveloping me with your enduring love and wholehearted support.

To my wonderful husband, Frank, for your endless love, humour and optimism which has sustained me throughout the entire study.

To my Saviour, for Your abundant blessings in my life and throughout this study.

It makes all the difference whether one sees darkness through the light

or brightness through the shadows.

David Lindsay 


\section{TABLE OF CONTINTS}

CHAPTER 1: INTRODUCTION 1

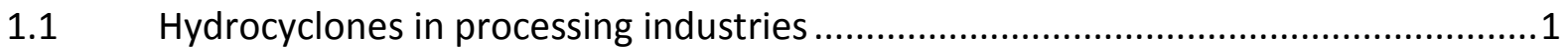

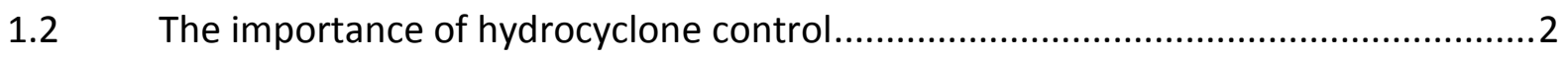

1.2.1 Hydrocyclone control in context of grinding circuits ............................................2

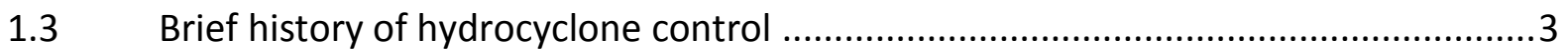

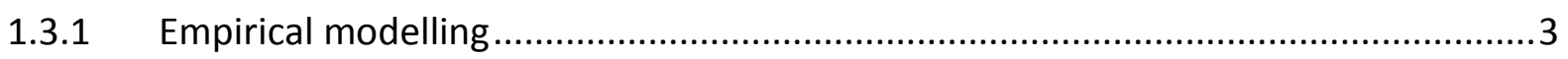

1.3.2 Theoretical/phenomenological modelling ...........................................................

1.3.3 Process state monitoring methods ..................................................................... 4

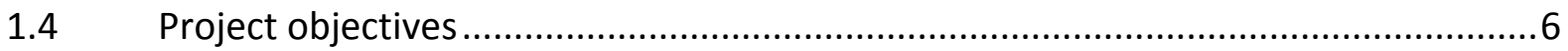

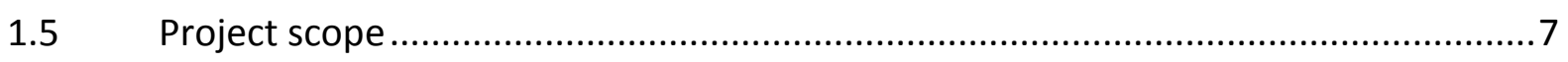

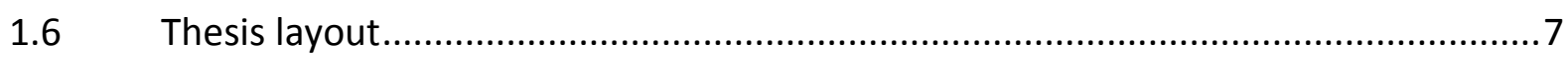

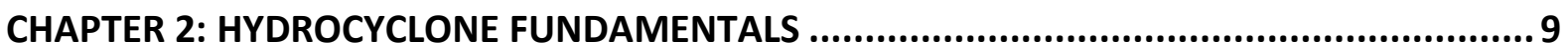

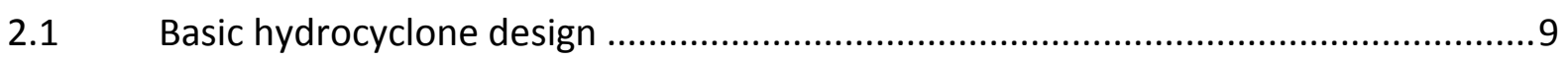

2.2 Operation and flow structure of hydrocyclone ................................................... 10

2.3 Variables affecting hydrocyclone performance ...................................................11

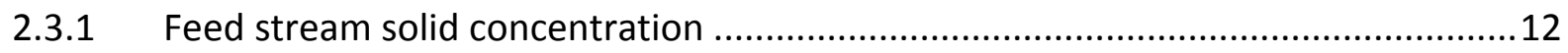

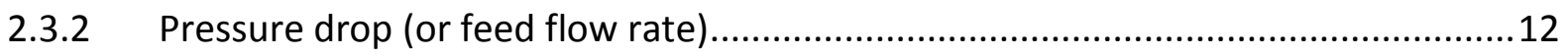

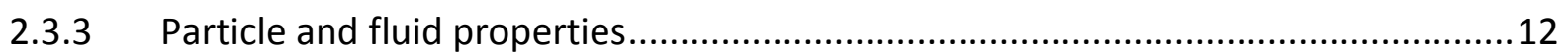

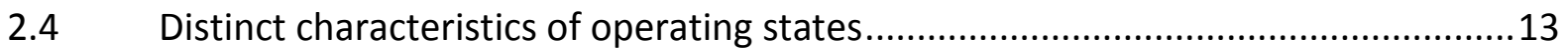

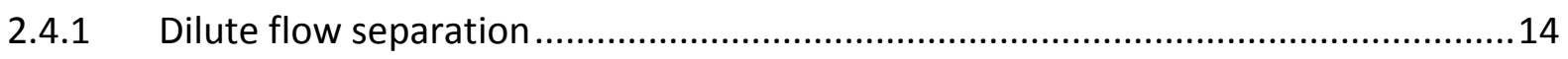

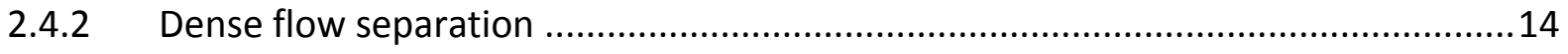

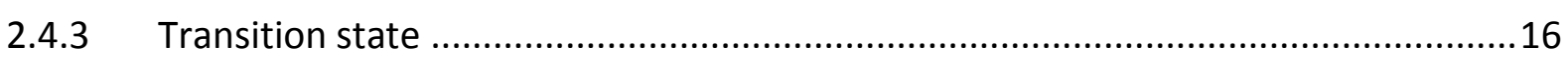

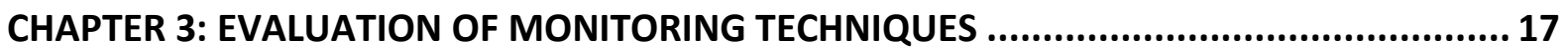

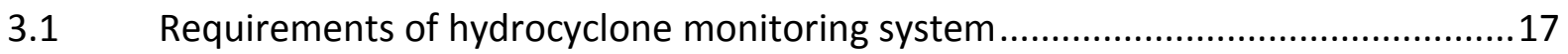

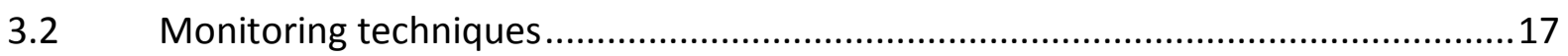

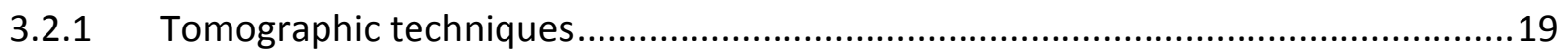

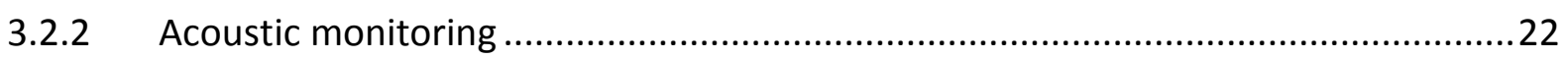

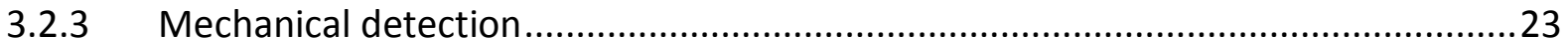

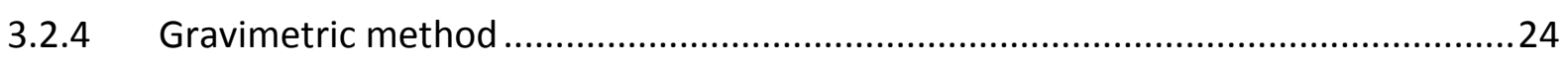

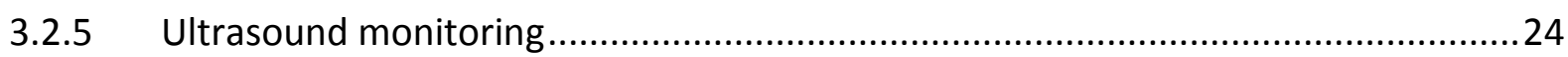

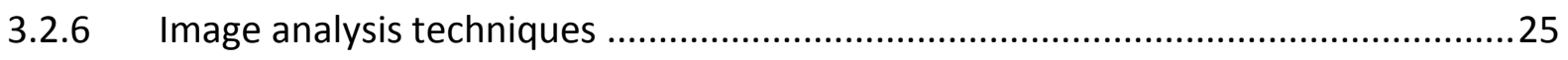

3.3 Motivation for image analysis as proposed monitoring technique ..........................26

3.4 Proposed entity for quantification of underflow shape ........................................28 


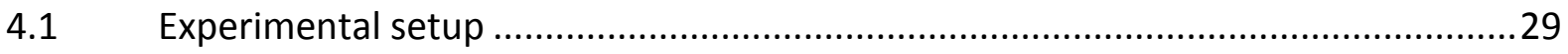

$4.2 \quad$ Experimental procedure

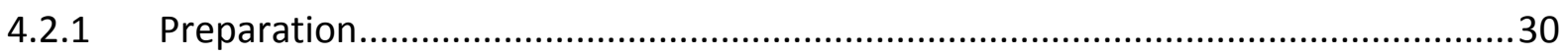

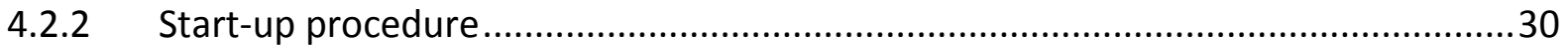

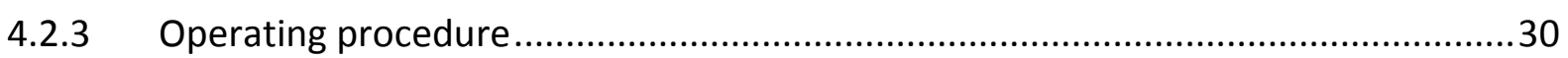

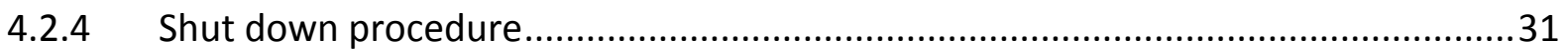

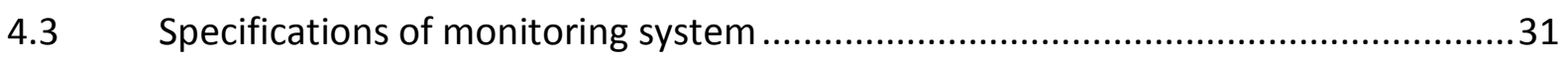

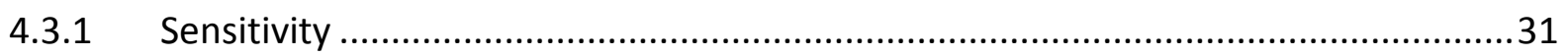

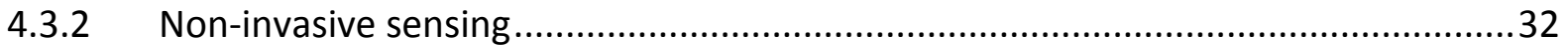

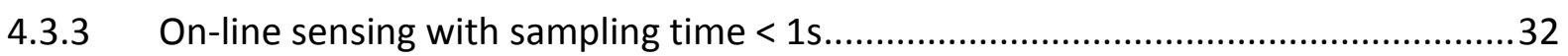

4.3.4 Robust sensors (insensitive to blinding and mechanical stress) ............................32

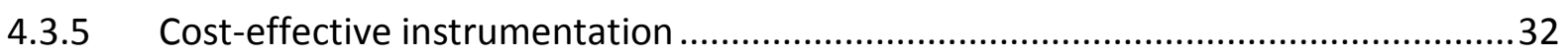

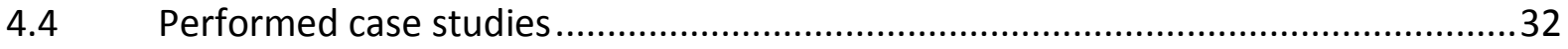

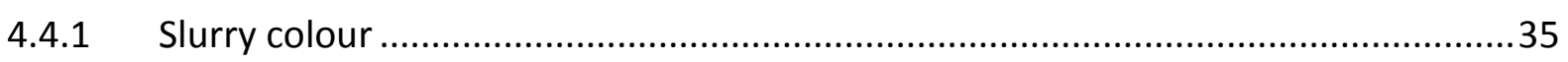

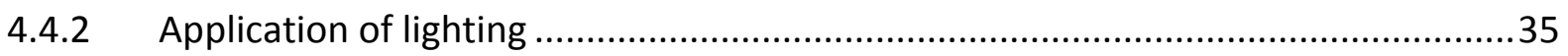

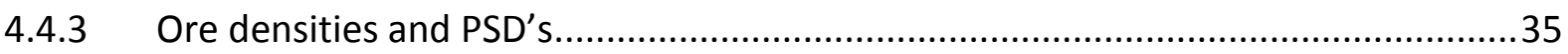

CHAPTER 5: IMAGE ANALYSIS AND DIAGNOSTIC MONITORING ..................................... 36

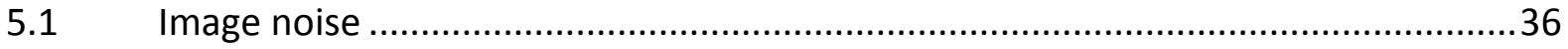

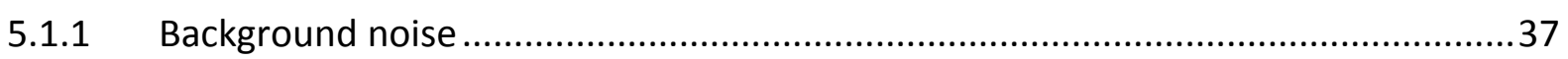

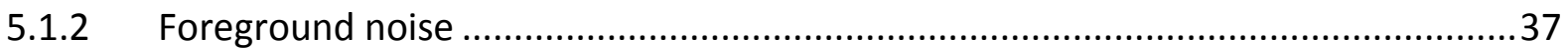

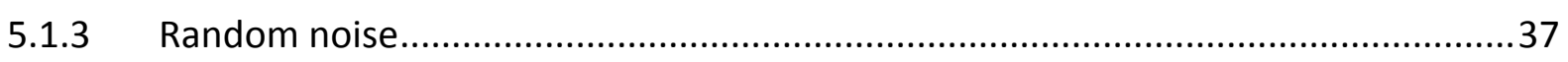

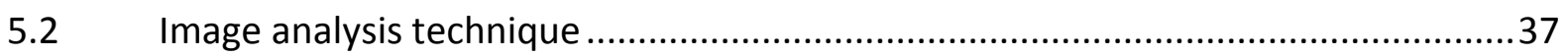

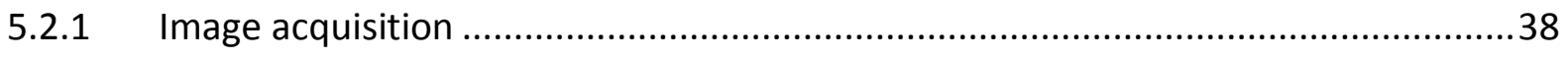

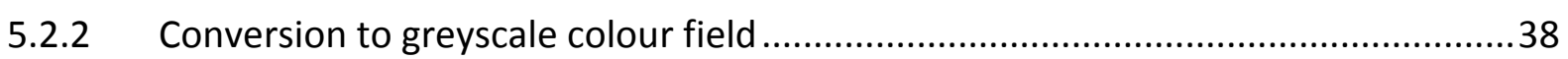

5.2.3 Image enhancement through contrast and brightness adjustments ......................39

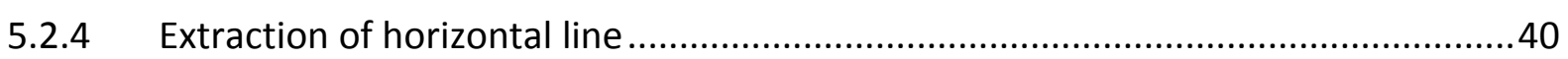

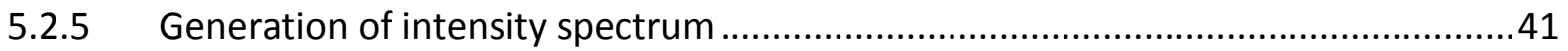

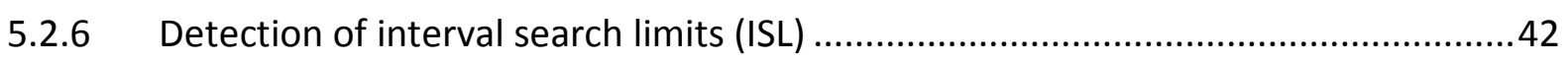

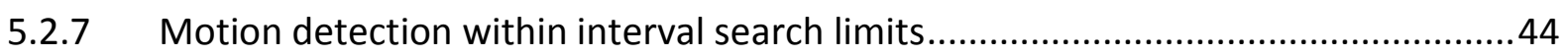

5.2.8 Extraction of underflow width .......................................................................... 44

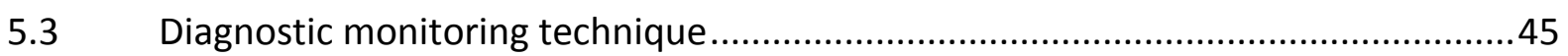

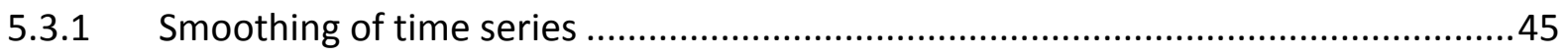

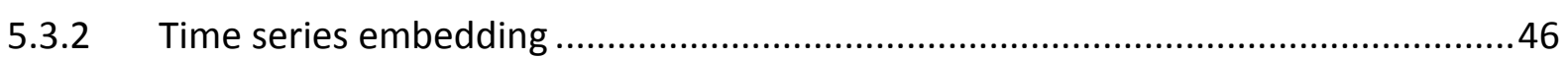

5.3.3 Support vector machines (SVM) …................................................................ 48

Conceptual calibration and on-line monitoring algorithms ....................................51 
6.1 Preliminary evaluation of image analysis technique .........................................56

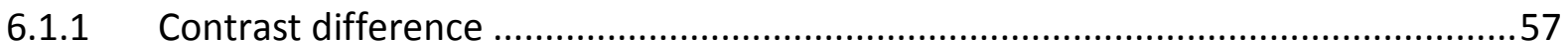

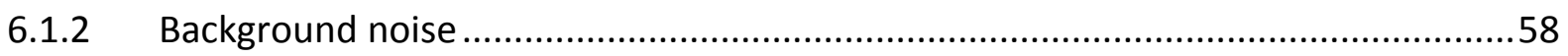

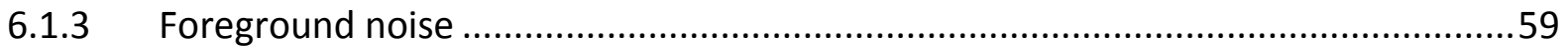

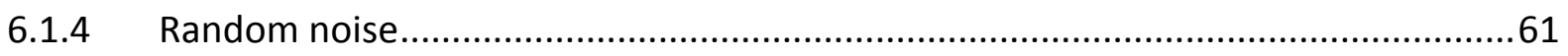

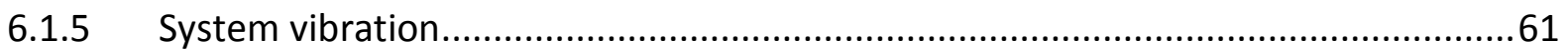

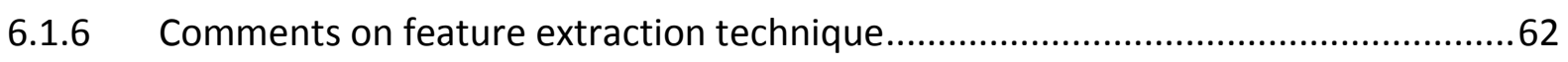

6.2 Underflow width results obtained through image analysis...................................62

6.2.1 Underflow width versus feed stream concentration ............................................62

6.2.2 Identification of operating states based on visual observations ..............................63

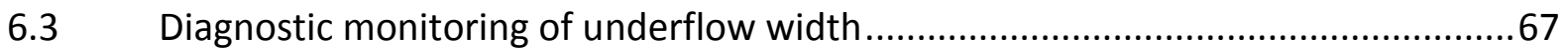

6.3.1 Selection of embedding lag, $\tau$, and phase space generation ...............................67

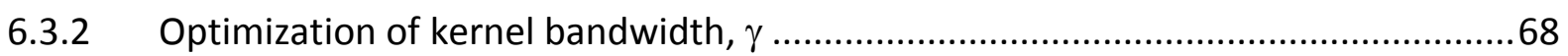

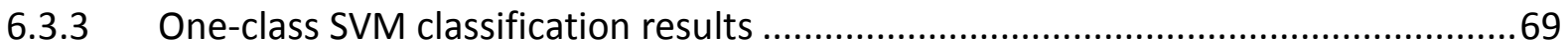

6.3.4 Comparison of one-class SVM and multivariate Gaussian distribution (MVGD)......73

6.3.5 Sensitivity of one-class SVM to embedding lag .................................................74

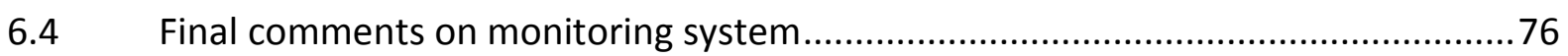

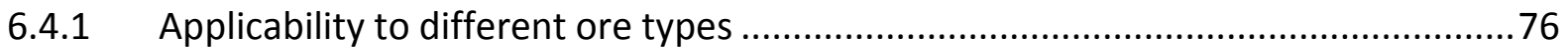

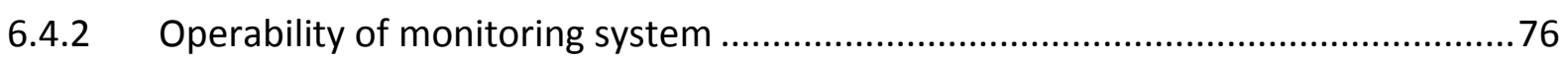

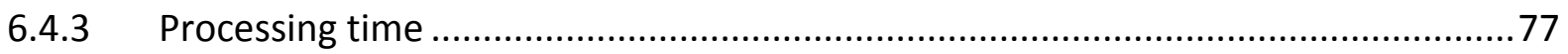

CHAPTER 7: CONCLUSIONS AND RECOMMENDATIONS ............................................... 78

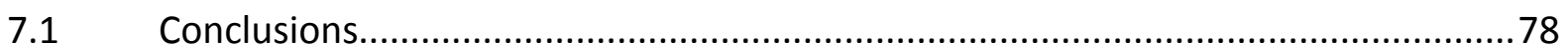

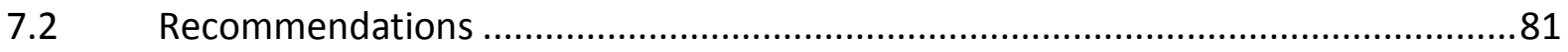

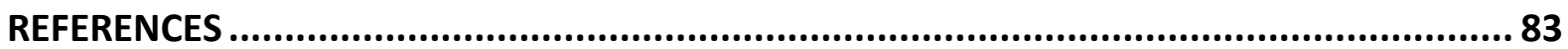

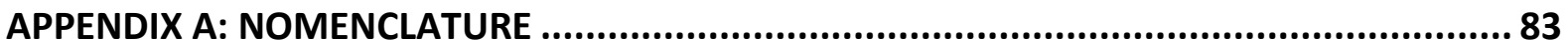

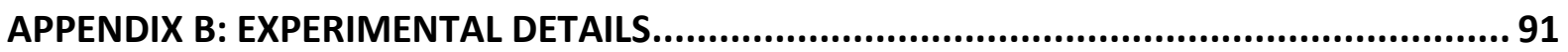

B-1 Stepwise instructions for experimental procedure ..........................................91

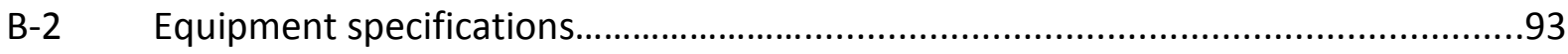

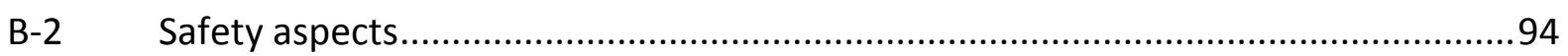

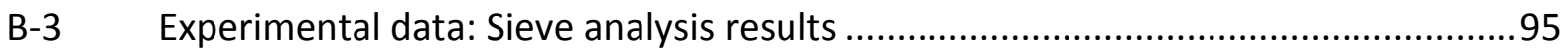

B-4 Experimental data: Feed stream concentration ................................................96 
APPENDIX C: IMAGE ANALYSIS AND DIAGNOSTIC MONITORING DETAILS. 101

C-1 Sample calculations: Image enhancement 101

C-2 Specified user inputs for different case studies................................................ 103

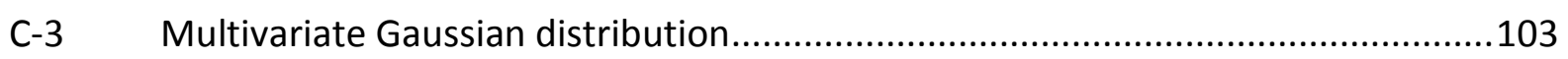

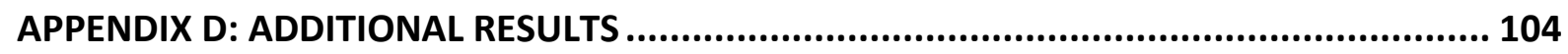

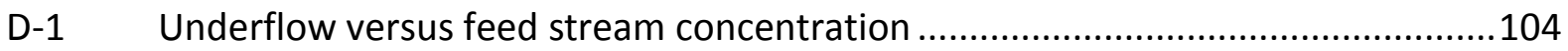

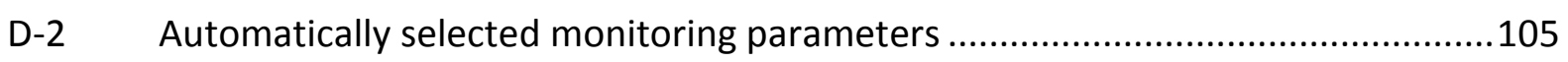




\section{CHAPTER 1: INTRODUCTION}

In this chapter the hydrocyclone is introduced as a valuable separation device. The importance of applying proper control to the device is emphasized and a brief overview of the various control methods is presented. Through evaluation of these methods, the potential of on-line monitoring of the hydrocyclone underflow by use image analysis is illustrated. The objectives, scope and thesis layout is furthermore presented.

\subsection{Hydrocyclones in processing industries}

Chemical and mineral processing entails the large scale conversion of raw materials into a valuable form. As most valuable materials do not exist naturally in a pure form, but rather as a mixture of two or more substances, chemical and physical separation processes are crucial to the processing industry (Taggart, 1950; Coulson et al., 1991). A hydrocyclone primarily functions as a physical separation device and is therefore a vital unit operation in most processing industries.

Since the first commercial implementation of a hydrocyclone in 1937 in the paper and pulp industry (Tarr, 1985), the device has found increased application in other fields such as mineral processing, petrochemistry, oil refineries, power generation, textile industry and food processing. These applications are broadly divided into various categories, based on the different separation requirements of processes (Svarovsky, 1984):

- liquid clarification;

- slurry thickening;

- solids washing;

- degassing of liquids;

- solids classification and sorting.

Except for its versatility, the widespread application of hydrocyclones is promoted by its simple design. The device does not involve any moving parts and consequently the costs involved with the procurement, instalment, operation and maintenance are relatively small. Compared to other separators, hydrocyclones are also very small in size and require very little support structures. Its small size further results in short residence times, which increase the speed of control (Kelly and Spottiswood, 1982; Svarovsky, 1984). 


\subsection{The importance of hydrocyclone control}

The main reasons for controlling process units are to increase process efficiency and to reduce variability in the end product. Proper control of a process could therefore lead to significant financial savings. This relates to a hydrocyclone as the device is continually subjected to varying feed conditions. Only a slight fluctuation in any of the feed stream properties could lead to a decrease in separation efficiency and a corresponding financial loss (Hou et al., 2002).

\subsubsection{Hydrocyclone control in context of grinding circuits}

The importance of hydrocyclone control can further be illustrated by considering its application in grinding circuits. This specific application is emphasized as it is one of the most common applications of the device in the mineral processing industry (Wills, 2003; Olson and Turner, 2002; Yalcin et al., 2003). This study will also focus on this specific usage.

An example of how a hydrocyclone is used in a grinding circuit is illustrated in figure 1-1. Ore from the crushing plant is subjected to grinding in a mill. Hydrocyclones are used to classify the mill product by removing the required size and returning the oversize material to the mill for regrinding.

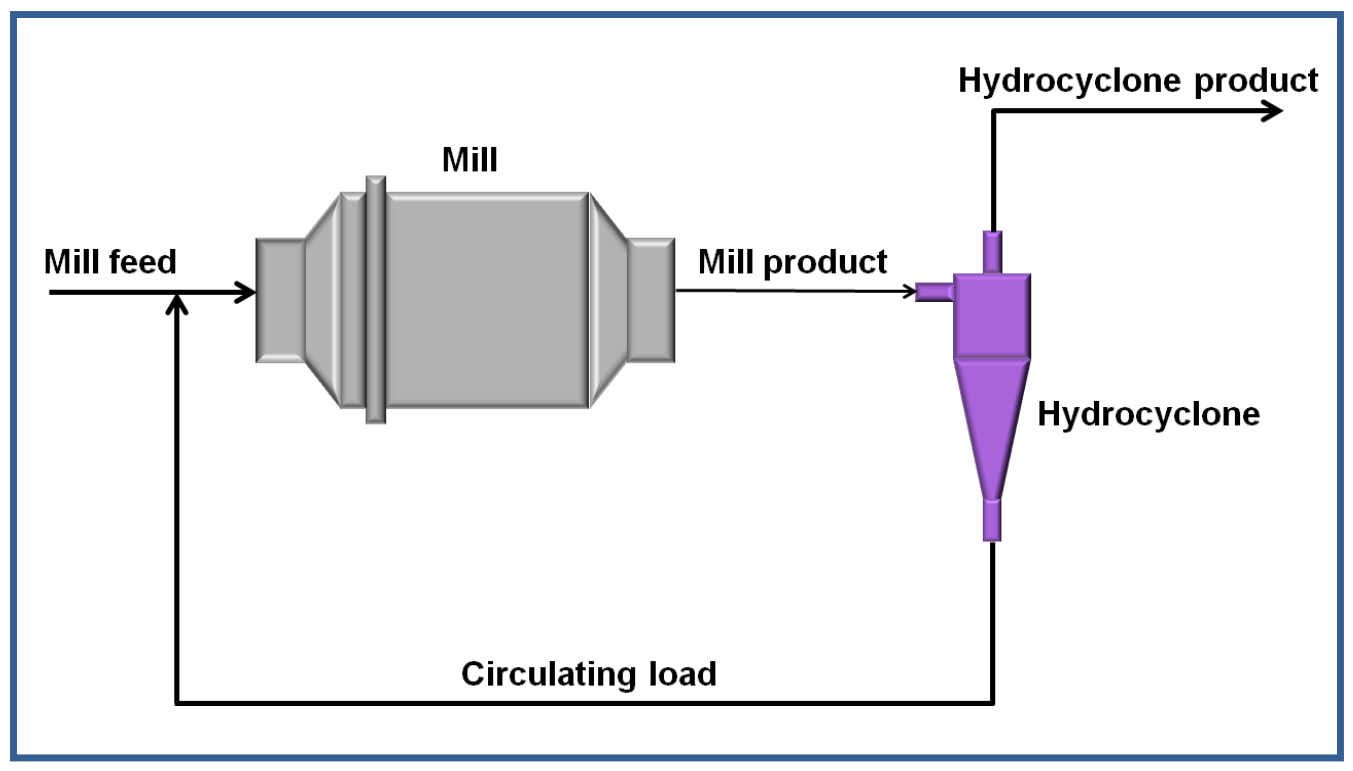

Figure 1-1: Diagram of closed grinding circuit

The operating costs associated with grinding circuits are very high due to the high energy requirements of the process. It has been estimated that comminution can consume from $65 \%$ to $80 \%$ of the total plant energy expenditure (Tromans and Meech, 2002). Due to increasing energy costs and a growing concern about the environmental impact of 
processes, it is important to minimize the energy consumption of grinding circuits through proper control.

Since hydrocyclones play a key role in the grinding process, the optimization and proper control of the device is essential. Overall grinding performance is interlinked with hydrocyclone performance in that it relies greatly on the ability of hydrocyclones to quickly adapt to the variations in the grinding process. By removing the correct size fraction from the grinding circuit, hydrocyclones could reduce the circulation load and therefore lower operating costs significantly. This would also avoid unnecessary energy expenditure on the overgrinding of material.

\subsection{Brief history of hydrocyclone control}

Despite the simple design of a hydrocyclone, its flow behaviour is quite complex and not yet fully understood (Dyakowski and Williams, 1993; Gutiérrez et al., 2000; Gupta et al., 2008). Complex features of hydrocyclone flow include its swirling motion, intense turbulence, the presence of an air core, particle laden flow and flow reversal effects (Williams et al., 1999; Nowakowski et al., 2004). Due to the fact that the flow dynamics are complex and largely unquantified, the prediction of hydrocyclone performance has come to rely strongly on modelling techniques.

The development of hydrocyclone modelling, as a means to control and optimise the device, is detailed in the following section. Shortcomings of these modelling techniques, which illustrate the need for an alternative control technique, are furthermore highlighted. This prompts the investigation of process state monitoring methods as an alternative for hydrocyclone control. A brief overview of the various sensing technologies used thus far in these monitoring methods is also provided.

\subsubsection{Empirical modelling}

The majority of models applied to hydrocyclones are empirical in nature (Dlamini et al., 2005). As these models are based on experimental observations, different data sets result in different equations for the same parameters (Narasimha et al., 2005). For hydrocyclones, empirical models relate separation parameters to device dimensions and slurry properties (Gupta et al., 2008).

Although empirical models have been developed and adapted to find widespread application in industry (Lynch and Rao, 1968; Marlow, 1973; Lynch and Rao, 1975; Plitt, 1976; Nageswararao, 1978; Castro, 1990), the technique suffers from inherit drawbacks: each model is only valid for the operating range, slurry properties and hydrocyclone geometry for which it was derived. Successful scale-up from empirical models is also, 
therefore, not guaranteed. This is emphasised by the fact that the majority of the models were developed for a certain range of hydrocyclone diameters $(100-380 \mathrm{~mm}$ ) (Lynch and Rao, 1975; Nageswararao, 1978; Plitt, 1976), without verified application to small diameter hydrocyclones (Napier-Munn et al., 1996).

\subsubsection{Theoretical/phenomenological modelling}

The deficiencies of empirical modelling have encouraged the development of fundamental models based on the physics of fluid flow. Important work in this field includes that of Hsieh and Rajamani (1991), Barrientos and Concha (1992) and Monredon et al. (1992). These models are solved either directly or through the use of commercial Computational Fluid Dynamic (CFD) packages (Nageswararao et al., 2004).

When compared to empirical techniques, theoretical/phenomenological models cover a greater operating range. The effect of geometry is furthermore accounted for in the model expressions (Hsieh and Rajamani, 1988). This characteristic renders these models as a useful design tool, allowing for the optimization of a device without having to build a prototype or performing experiments (Dlamini et al., 2005).

However, the application of these techniques in CFD modelling is hindered by the fact that hydrocyclone flow consists of complex physical phenomena which are not yet fully understood (Nowakowski et al., 2004). Models used thus far are therefore based on greatly simplifying assumptions. Three-dimensional modelling has only been attempted in recent years, where the majority of the studies focused on simulating the water phase only and very few attempts were made to incorporate solid particles (Slack and Wraith, 1997; Narasimha et al., 2005; Cristea et al., 1994). Due to these limitations, the soundness of all results also still requires validation through experimental work (Wesseling, 2001).

Another disadvantage of CFD modelling is the amount of computer power and time that is required to obtain a solution (Nageswararao et al., 2004). It is expected that the execution time of CFD hydrocyclone models will only be reduced to practical values (1-2 s) in about twenty years (Nageswararao et al., 2004). Widespread use of this modelling technique in industry is further hindered by the shortage of experts in this specialised field.

\subsubsection{Process state monitoring methods}

The restrictions set by current modelling techniques necessitate an alternative approach to hydrocyclone control. Process state monitoring methods, by use of sensing techniques, proves to be a viable option in this regard. The advantage of such on-line monitoring techniques as a means to control is that it provides dynamic and real-time information of the process, allowing immediate evaluation and possible corrective action to take place. It is 
also expected that such monitoring techniques could be used to shed light on the complex internal flow features and serve as validation for CFD simulations (Wang et al., 2009).

As the name suggests, process state monitoring methods rely on the identification of the hydrocyclone operating state. ${ }^{*}$ The underlying approach of this method is based on the fact that the operating state can be used to interpret and improve the device's performance, since it gives a qualitative indication of separation efficiency (Neesse, Golyk et al., 2004; Neesse, Schneider et al., 2004). For example, the operating state called dense flow separation indicates inefficient separation.

The majority of process state monitoring methods used thus far, focus on the determination of the operating state from the shape of the air core. Sensing techniques employed in these methods include electrical impedance-, electrical resistance- and ultrasound tomography (Gutiérrez et al., 2000; Williams et al., 1999; Schlaberg et al., 2000; Dyakowski et al., 2000). Other monitoring methods concentrate on determining the operating state from the internal solids concentration (Schweitzer, 1972; Galvin and Smitham, 1994; Gutiérrez et al., 2000; Hou et al., 2002). A third group of monitoring methods rely on detection of the operating state from the shape of the underflow discharge. This is done either through mechanical detection (Hulbert, 1993), or through sensing techniques such as acoustic monitoring (Neesse, Schneider et al., 2004).

Although the mentioned techniques have all been applied with varied success, none has found widespread adoption yet. The work of Neesse, Schneider et al. (2004) suggests that the limited application of these methods might be attributed to the following factors:

- the monitoring methods rely on complex measuring and processing principles;

- relatively expensive equipment is required;

- equipment and sensors lack robustness;

- significant alterations to the process and hydrocyclone are required;

- the sensing method is invasive to the process.

The work of Petersen (1996) presented an alternative monitoring technique: hydrocyclone monitoring through image analysis of the underflow. The image-based approach proved advantageous compared to other techniques considering the minor modifications required, low equipment cost and non-invasive approach. Although the study provided positive results, complications were experienced with the collection and analysis of a continuous data set.

\footnotetext{
Process state monitoring should be distinguished from the conventional monitoring of operating variables (such as pressure, concentration, particles size etc.) which incidentally also forms the basis for empirical modelling.
} 
A more recent study incorporating image analysis is that of Neesse, Schneider et al. (2004) which involves measuring the underflow by use of a laser-optical measuring device. Preliminary results from this study confirmed that the underflow shape can be used to identify the operating state.

The advantages put forward by Petersen (1996) and the results obtained by Neesse, Schneider et al. (2004), suggest that image analysis of the underflow is a viable alternative. The problems experienced by Petersen (1996), in terms of computationally intense nature of monitoring method, could also now be overcome due to the advances that has been made in visual and computer technology. Although the method used by Neesse, Schneider et al. (2004) provides a good basis to work from, it also leaves scope to explore image-based monitoring in terms of processing techniques and fault detection. In light of these findings, the image analysis of hydrocyclone underflow is investigated as a monitoring technique.

It should be noted that a detailed evaluation of the various monitoring methods is an expected outcome of this study. The brief evaluation presented in this section, is therefore expanded in Chapter 3.

\subsection{Project objectives}

The main objective of this study is to explore the feasibility of image analysis of hydrocyclone underflow as a monitoring technique. In support of this goal, the following secondary objectives are specified for the execution and evaluation of the work:

- Perform a literature review of sensing techniques which have previously been employed and evaluate the use of image analysis by comparing its advantages and disadvantages to that of previous techniques.

- Develop an image analysis technique which provides a satisfactory account of variation in underflow shape.

- Investigate how image quality affects the image analysis process and determine to what extent images can be improved, if necessary.

- Determine whether underflow data, obtained through image analysis, can be used to identify the various operating states, specifically the troublesome dense flow separation.

- Investigate the applicability of the technique to different ore types.

- Evaluate the practicality of the developed monitoring system with reference to the hydrocyclone monitoring requirements set by Neesse, Schneider et al. (2004). 


\subsection{Project scope}

As stated in section 1.4, the objective of the study is to explore the feasibility of image analysis of the underflow as a monitoring technique. The study is, however, limited to a proof-of-concept investigation, and does not incorporate the development of a prototype device.

The study will also focus on the specific use of a hydrocyclone as a separator in grinding circuits. This implies that the only application which will be considered is that of ore sorting and sizing. Other applications such as degassing of liquids, liquid clarification, slurry thickening and solids washing are therefore excluded. Investigations are further limited to individually operated hydrocyclones (with atmospheric underflow discharge) and do not incorporate hydrocyclone batteries.

\subsection{Thesis layout}

The various topics addressed in the thesis are presented in the following manner:

Chapter 2 presents the fundamental theory behind the operation of the hydrocyclone. In chapter 3 , the evaluation of the various monitoring techniques is expanded and the promising features of the chosen technique highlighted. The details of the experimental methodology are covered in chapter 4 . Chapter 5 describes the developed image analysis technique used to extract underflow data. Subsequent diagnostic monitoring techniques are also described. The final results, and interpretation thereof, are provided in chapter 6 . Finally, the conclusions and recommendations are presented in chapter 7. 


\section{CHAPTER SUMMARY}

Hydrocyclones play a crucial role in processing industries and it is therefore important that proper control is applied to the device. Although the device is simple in operation, the fluid flow is very complex. This complicates the control of the device significantly. Consequently, most of the approaches to control the hydrocyclone are based on empirical and fundamental modelling of the device. Since these models are limited to certain operating ranges and device geometries, monitoring techniques are considered a promising alternative method of control. The majority of monitoring techniques used thus far involve sensing methods that are either expensive, lack robustness, require significant process alterations or intrusive to the process. Image analysis of the underflow, however, makes use of a sensing method which is advantageous with regard to all of these aspects. The purpose of the project is to evaluate the viability of image analysis of the underflow as a hydrocyclone monitoring technique. 


\section{CHAPTER 2: HYDROCYCLONE FUNDAMIDTTALS}

The intention of this chapter is to supply the fundamental concepts necessary to understand hydrocyclone operation and performance within context of this study. The basic design, operation, and flow structure of the device is therefore described. Hydrocyclone performance is further detailed by investigating the effect of operating variables and the connection with the various operating states.

\subsection{Basic hydrocyclone design}

The body of a hydrocyclone consists of two parts: a conical section and a cylindrical shaped part. A feed inlet is connected tangentially to the top of the cylindrical section. This inlet can be either circular or square in shape.

At the tip of the conical section there is an opening through which the underflow stream discharges, also referred to as the spigot. An overflow pipe is mounted on the top centre of the cylindrical section. The vortex finder is an extension of the overflow pipe which projects into the hydrocyclone and serves to prevent short-circuiting of the feed into the overflow. A schematic of the typical hydrocyclone design is illustrated in figure 2-1.

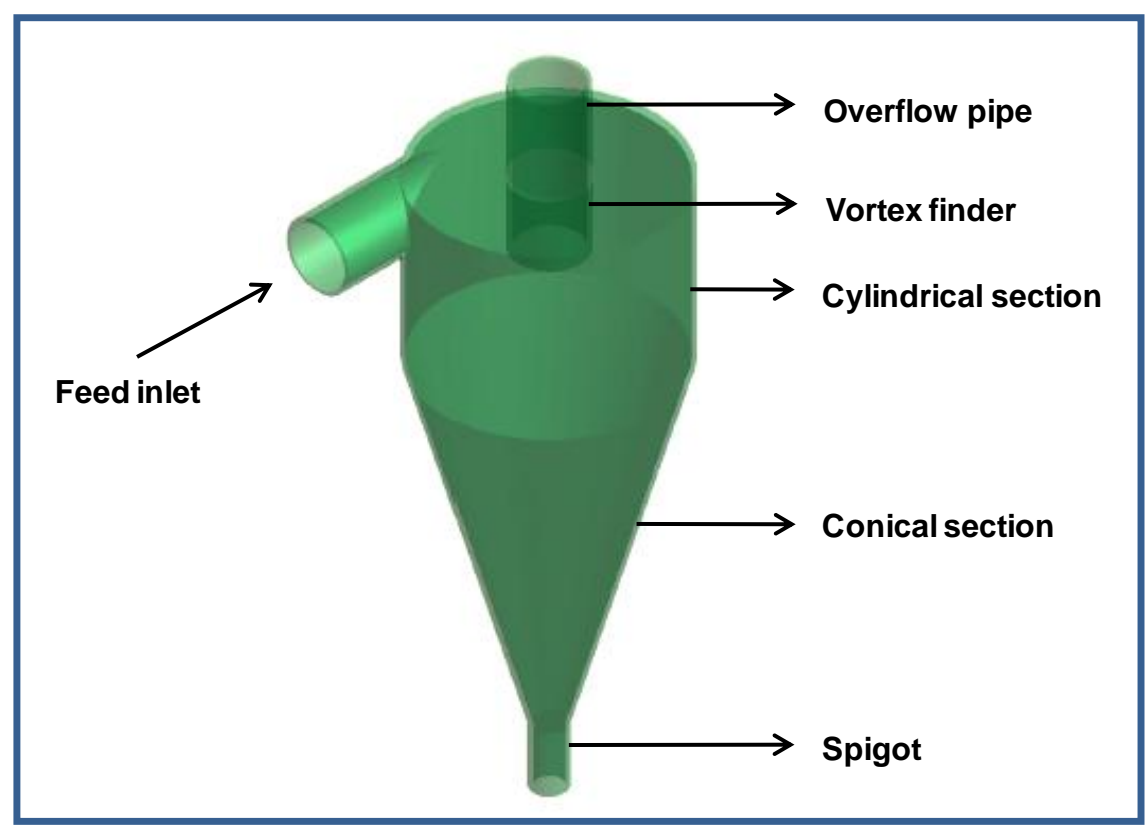

Figure 2-1: Typical design of hydrocyclone body 


\subsection{Operation and flow structure of hydrocyclone}

Two products are formed as result of separation in a hydrocyclone: a coarse product (particles in the underflow stream) and a fine product (particles in the overflow stream). Separation of the product streams is accomplished through centrifugal sedimentation: a vortex motion is established by the fluid which exerts centrifugal forces on the suspended particles, thus achieving separation based on size, shape and density. No moving parts are required for the formation of a vortex as the swirling motion is accomplished by the tangential injection of the feed inlet.

Particles in a hydrocyclone are subjected to an acceleration force (consisting of a gravitational and a centrifugal component) as well as a drag force. It is only necessary to consider the centrifugal and drag forces as the gravitational component is usually neglected (Svarovsky, 2000). Figure 2-2 illustrates the force balance of a particle in a hydrocyclone.

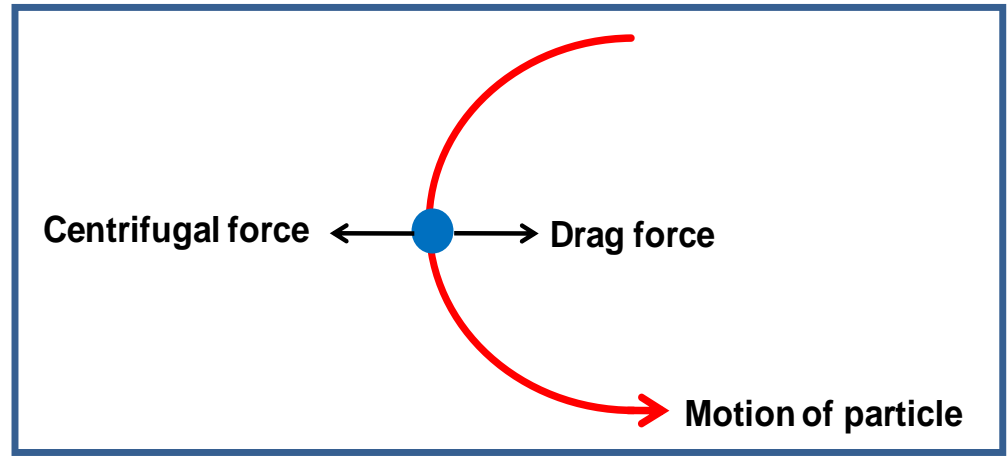

Figure 2-2: Force balance of orbiting particle in hydrocyclone

The centrifugal force accelerates the settling rate of the particles, allowing particles of different sizes and densities to be separated. A faster settling particle is subjected to a greater centrifugal force and drifts radially outwards to a lower velocity region. These particles are transported to the wall of the hydrocyclone and move within a downward vortex to the spigot. At the spigot opening the particles are ejected as part of the underflow or coarse product stream.

For slower settling particles the drag force exceeds the centrifugal force. As result, the particles are moved to a low pressure region that stretches across the centre of the hydrocyclone. From here the particles move through an upward vortex motion. When the vortex finder is reached, the particles exit through the overflow or coarse product stream. The two opposing vortex motions, that enable particle separation, are illustrated in figure 2-3. 


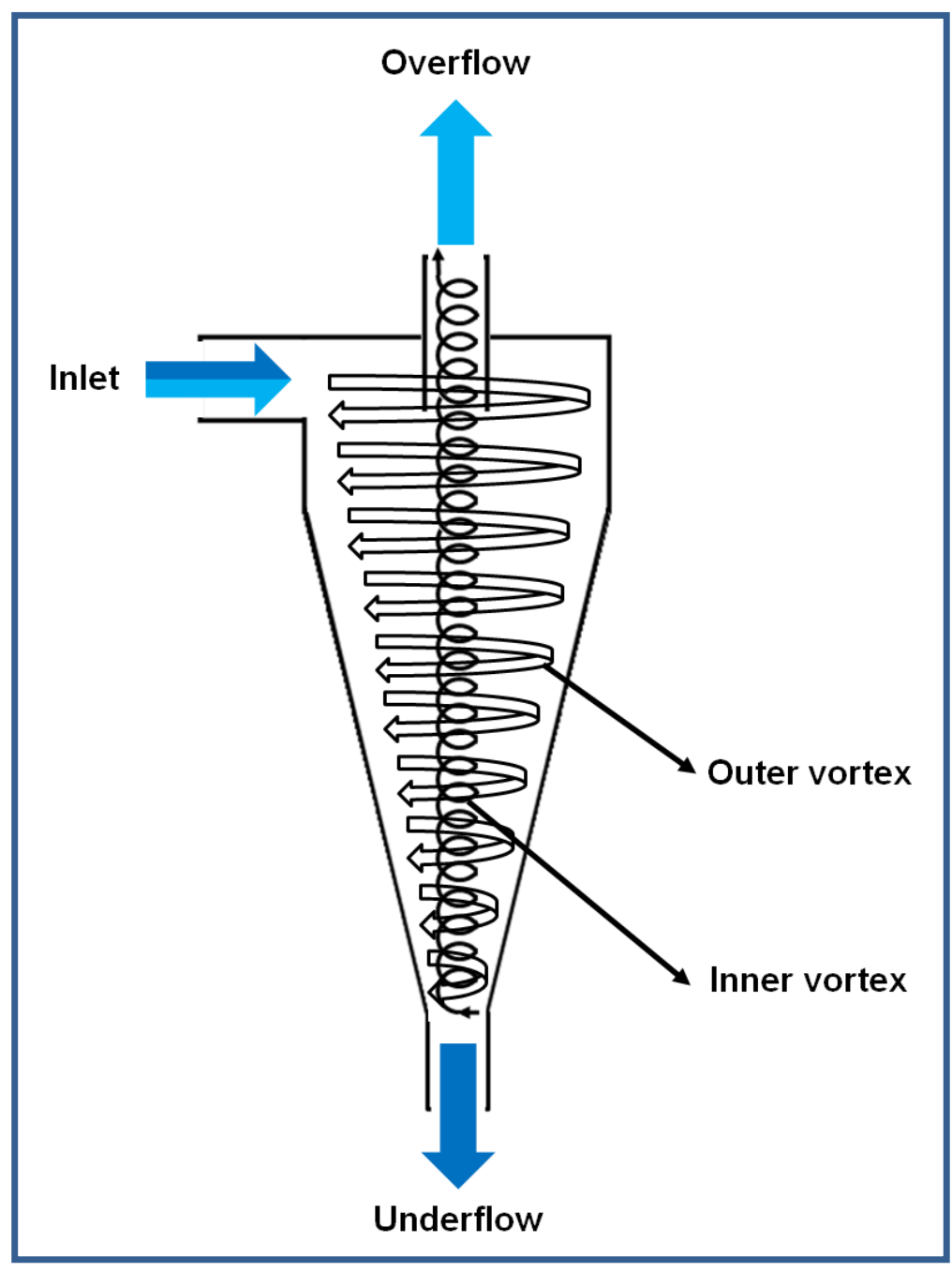

Figure 2-3: Vortex flow within a hydrocyclone (redrawn from Svarovsky, 1984)

Another feature of the hydrocyclone flow structure is a centrally developed air core. Air core development is induced by strong vortex motions in the central axis region which lead to pressure reduction and, in turn, could initiate a gas-liquid interface (Svarovsky, 1984; Dlamini et al., 2005). The air which establishes air core development is supplied through the spigot and as part of the feed stream (Neesse and Dueck, 2007).

\subsection{Variables affecting hydrocyclone performance}

Hydrocyclone performance is influenced by design variables and operating variables. Design variables are dependent on the hydrocyclone geometry and therefore include the hydrocyclone size, hydrocyclone shape and the feed, overflow and underflow orifice sizes (Kelly and Spottiswood, 1982). Operating variables, on the other hand, are defined as variables dependent on the feed stream. The hydrocyclone operating variables incorporate the following (Kelly and Spottiswood, 1982; Svarovsky, 1984): 
- feed stream solid concentration;

- $\quad$ pressure drop (or feed flow rate);

- $\quad$ particle and fluid properties.

As part of the experimental work of this study, changes in operating states and separation efficiency are brought about by changing the feed stream solid concentration (refer to section 4.2). Although feed stream solid concentration is the only operating variable that is manually adjusted, the following section will briefly detail the influence of all operating variables on hydrocyclone performance, since these variables are all closely interrelated (Svarovsky, 1984). Investigations into the influence of design variables are not included as each experiment is performed on a hydrocyclone with fixed dimensions.

\subsubsection{Feed stream solid concentration}

An increase in the feed stream concentration leads to a decrease in pressure drop, a decrease in separation efficiency and a coarser cut-size (size at which particle has $50 \%$ chance to report to either the overflow or underflow). This is mainly due to the effective increase in viscosity and degree of hindered settling. Another possible reason might be the overloading of the underflow orifice. This, in turn, increases the amount of particles that short-circuit through the overflow orifice (Braun and Bohnet, 1990).

\subsubsection{Pressure drop (or feed flow rate)}

A direct relationship exists between the feed flow rate and the static pressure drop measured between the inlet and the overflow. The interdependency of these two variables is illustrated by the fact that an increase in pressure drop leads to an increase in feed flow rate (Svarovsky, 1984; Wills, 2003).

As the pressure drop, and hence the flow rate, is increased, greater centrifugal forces are experienced. Consequently, the separation efficiency is increased, moving the performance curve to finer sizes and ultimately leading to a reduction in cut size. As result, the overflow becomes cleaner and the concentration of the underflow increases.

\subsubsection{Particle and fluid properties}

Particle and fluid properties that affect hydrocyclone performance considerably include density and viscosity, particle size and distribution, particle shape and the presence of chemical agents. The latter is not conventional practice and therefore not discussed in further detail. 


\section{(a) Density and viscosity}

As mentioned in section 2.2, it is required that there should be a density difference between the particles (solids or liquid droplets) and the continuous phase to achieve separation. Without this density difference, the buoyancy force acting on a particle would be equal to the centrifugal force, inhibiting separation (Svarovsky, 1984).

An investigation by Possa and Lima (2000) show that slurry viscosity influences hydrocyclone performance in the following manner: for an increase in solids content (and hence viscosity) the cut-size increases, while the opposite also holds for a decrease in viscosity.

\section{(b) Particle size distribution}

Particle size and distribution play an insignificant role at low concentrations. At higher concentrations, greater than one or two mass percent, the particle size and distribution start to affect the cut-size as a finer particle size leads to an increase in cut-size (Svarovsky, 1984).

\section{(c) Particle shape}

Wills (2003) and Svarovsky (2000) emphasize the importance of considering the role of the particle shape. Mica, a relatively coarse particle, has often appeared in the overflow, rather than the underflow as expected, due to the particle's flat shape.

\subsection{Distinct characteristics of operating states}

Schubert and Neesse $(1973 ; 1980)$ and Neesse et al. (1986; 1991) characterised the operating states of a hydrocyclone by defining two variants: dilute flow separation and dense flow separation. A third state, which represents the transition between the diluteand dense flow variants, was later identified and is referred to as the transition state by Neesse, Schneider et al. (2004). Typical features, which serve as indication of the various operating states, include:

- $\quad$ sediment mass stored in the hydrocyclone's conical section;

- extent of air core formation;

- shape of the underflow discharge.

In terms of these features, the distinct characteristics of each operating state are identified and described in the following section. A visual comparison of the operating states is also presented in figure $2-4$. 


\subsubsection{Dilute flow separation}

For low feed concentrations a high rotational spray discharge is achieved. Typically, this type of discharge is referred to as 'spray' or 'umbrella' discharge.

During this state, solid particles generally stay in suspension in the hydrocyclone body. Consequently a very thin sediment layer forms along the wall of the hydrocyclone. A fully developed air core is maintained through centrifugal forces along the central axis of the device and opens to the atmosphere through the centre of the discharge stream (Svarovsky, 1984).

As result of the air core, more fines are ejected in the underflow stream with low solid concentrations. Such high recoveries of fine material are undesired.

\subsubsection{Dense flow separation}

If the solids content in the underflow is increased, due to either an increase in feed concentration or separation efficiency, or when the size of the spigot orifice is reduced, a rope shape discharge is obtained. In this instance, the discharge is a stream of similar diameter as the spigot opening (Wills, 2003). Commonly, this type of discharge is referred to as 'roping'. This configuration is established as boundary layers, which direct flow towards the underflow discharge, eventually join (Svarovsky, 1984). Consequently, the discharge stream is expelled at low rotational speed.

With continuation of roping conditions the underflow orifice becomes burdened with excess material and more solids are stored in the conical section of the hydrocyclone. The air core cannot pass through the body of the hydrocyclone and therefore oscillates intensively or disappears completely. Ultimately, the increase in sediment mass leads to the short-circuit of solid material and an associated loss in efficiency. Further operation could result in a snake-like discharge, with the danger of causing blockages. According to Svarovsky (1984) blocking can also occur before this point is reached.

Although a discharge stream of high solid content is desired, roping conditions are generally avoided due to the instabilities and danger of blocking. 


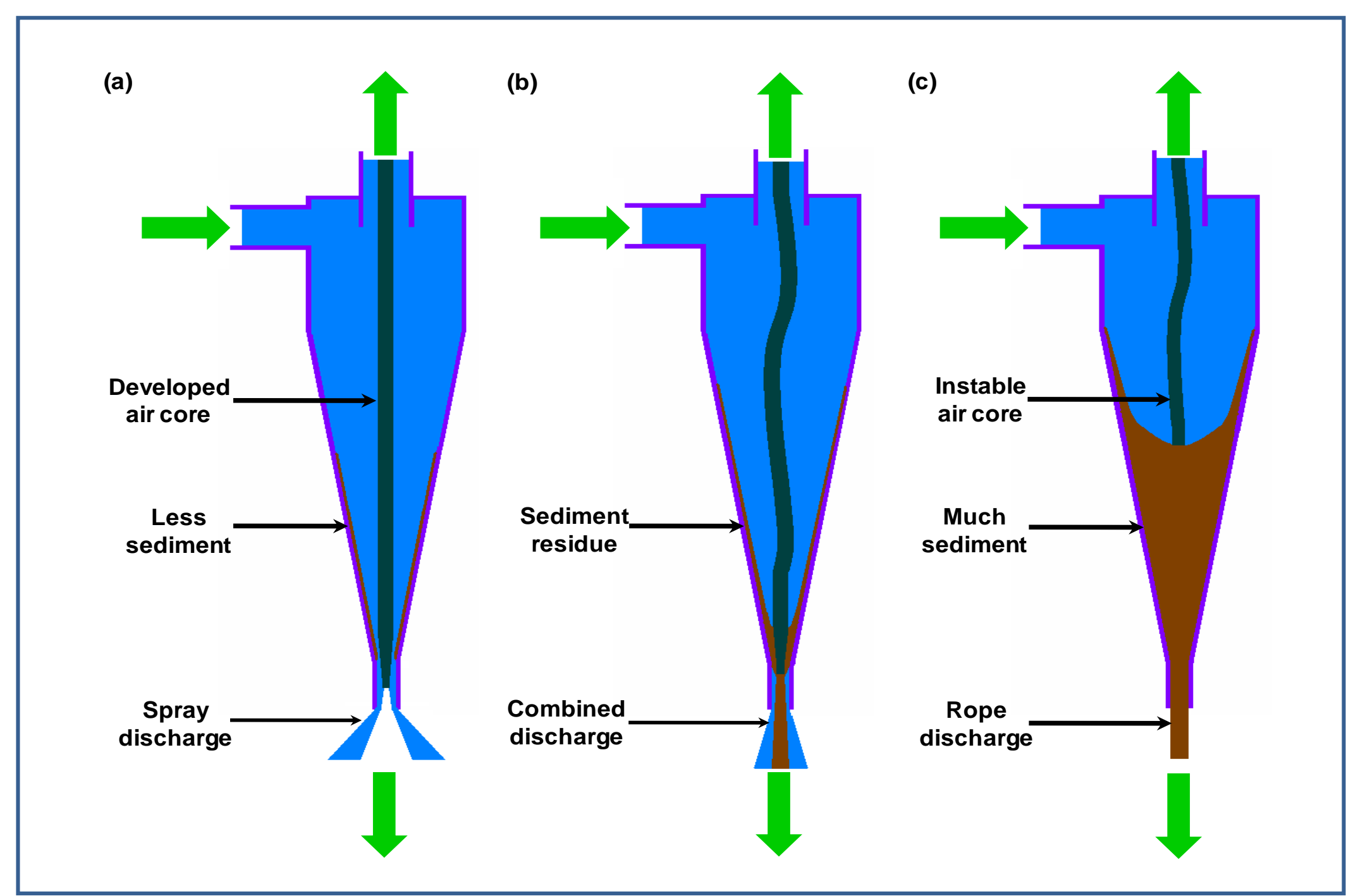

Figure 2-4: Operating states of a hydrocyclone: (a) dilute flow separation, (b) transition state and (c) dense flow separation (redrawn from Neesse, Schneider et al., 2004) 


\subsubsection{Transition state}

The transition state is signified by rapid changes between rope and spray discharge. As result, the underflow discharge is presented as a combination of these two discharge types, and is aptly described as 'combined' discharge. Although the extent of air core development is an improvement on the dense flow separation state, the maintenance of a fully developed core is impeded by the sediment residue in the hydrocyclone body.

Separation during this state is most favourable because it holds both high solids recovery and high solid underflow concentration as advantages (Neesse, Schneider et al., 2004). To exploit these optimal separation conditions, the identification and subsequent control of the transient operating state is of utmost importance.

\section{CHAPTER SUMMARY}

A hydrocyclone separates particles based on size and density differences under the effect of centrifugal forces. These centrifugal forces also lead to the formation of two opposing vortices which, together with the air core, complicates the device's internal flow structure significantly. The performance is greatly influenced by the operating variables which include feed stream solid concentration, pressure drop (or feed flow rate) and particle and fluid properties. Depending on these operating variables, the hydrocyclone will operate in one of three operating states: dilute flow separation, dense flow separation or transition state. Of these three operating states the transition state is the most favourable state as it provides the best separation conditions. Dense flow separation or roping, on the other hand, is the least desired due to general instability and the increased chance of blocking. 


\section{CHAPTIS 3: EVALUATION OF MONITORING TECHINIOUIES}

An evaluation of the various monitoring techniques used in the past, is presented as an outcome of this study in the following chapter. These techniques are compared in terms of the monitoring setups, methodology, sensing capabilities and potential to identify the various operating states. Advantages and disadvantages mentioned in literature are also stated. The results of this evaluation further serve as motivation for the proposed technique.

\subsection{Requirements of hydrocyclone monitoring system}

According to Neesse, Schneider et al. (2004), the following aspects concerning sensing capabilities should be considered for an on-line hydrocyclone monitoring system:

- sensitivity;

- non-invasive sensing;

- sampling time smaller than $1 \mathrm{~s}$;

- sensor robustness (resistance to blinding and mechanical stress);

- cost effective equipment.

In addition, it is important to consider the complexity of the monitoring method, in terms of its usability and required data analytical techniques. It is further preferred that the installation of such a system would not require extensive modifications to existing setups. The monitoring system should also require very little maintenance and replacement.

\subsection{Monitoring techniques}

Various monitoring techniques have been implemented in the past, with varying success. However, none has found broad adoption yet. The reason for this is that most of the techniques do not comply with the hydrocyclone monitoring requirements, stipulated in section 3.1.

In the following section, some of the different techniques investigated during the past, are evaluated. A summary of the reviewed techniques is presented in table 3-1. For the purpose of comparison, the monitoring setups, methodology and sensing capabilities are described as presented in literature. The degree to which these techniques could identify the operating states is also highlighted, together with mentioned shortcomings and advantages. This evaluation therefore also serves as a platform for the development of the monitoring technique proposed in this study. 
Table 3-1: Various hydrocyclone monitoring techniques investigated in the past

\begin{tabular}{|c|c|c|c|}
\hline Monitoring technique & Sensory system & Measured feature & References \\
\hline \multirow{4}{*}{ Tomography } & Electrical resistance tomography & $\begin{array}{l}\text { Air core shape and size } \\
\text { Internal density distribution }\end{array}$ & $\begin{array}{l}\text { - Williams, Ilyas et al., } 1995 \\
\text { - Williams et al., } 1999 \\
\text { - Wyakowski et al., } 2000 \\
\text { West et al., } 2000\end{array}$ \\
\hline & Electrical impedance tomography & $\begin{array}{l}\text { Air core shape and size } \\
\text { Internal density distribution }\end{array}$ & $\begin{array}{l}\text { - Williams et al., } 1997 \\
\text { - Gutiérrez et al., } 2000\end{array}$ \\
\hline & X-ray tomography & Internal density distribution & - Galvin and Smitham, 1994 \\
\hline & Ultrasound tomography & Air core size and position & - Schlaberg et al., 2000 \\
\hline Acoustic monitoring & Acoustic emission & $\begin{array}{l}\text { Feed pressure } \\
\text { Feed solids concentration } \\
\text { Feed flow rate }\end{array}$ & $\begin{array}{l}\text { - Williams et al., } 1996 \\
\text { - Hou et al., } 1998 \\
\text { Hou et al., } 2002\end{array}$ \\
\hline Ultrasound monitoring & Ultrasonic emission & Discharge oscillation & - Olson et al., 2006 \\
\hline \multirow{3}{*}{ Image analysis } & \multirow{3}{*}{ Optical emission } & Air core shape and size & - Castro et al., 1996 \\
\hline & & Discharge spray shape & - Neesse, Schneider et al., 2004 \\
\hline & & Discharge spray angle & $\begin{array}{l}\text { - Petersen et al., } 1996 \\
\text { - Van Deventer et al., } 2003\end{array}$ \\
\hline
\end{tabular}




\subsubsection{Tomographic techniques}

\section{(a) Electrical resistance tomography (ERT)}

Williams et al. (1999) applied ERT to a hydrocyclone to investigate its potential as a monitoring method. ERT is used to obtain a cross-sectional image of the electrical conductivity distribution in a process vessel (Industrial Tomography Systems plc., 2010). As reported in a preliminary study, ERT images typically illustrate the extent of air core development (Williams, llyas et al., 1995). The application was later extended to also obtain information regarding the amount of sediment stored by yielding internal density distributions (Williams et al., 1999; Dyakowski et al., 2000; West et al., 2000).

In the Williams et al. (1999) study, various physical alterations had to be made to the setup for sensor installation. This involved fabricating eight planes of 16 electrodes in a resin mould followed by the retrofitting thereof on a commercial hydrocyclone (refer to figure 3-1 (a)). Tests were performed in a laboratory, pilot scale plant as well as an operating plant.

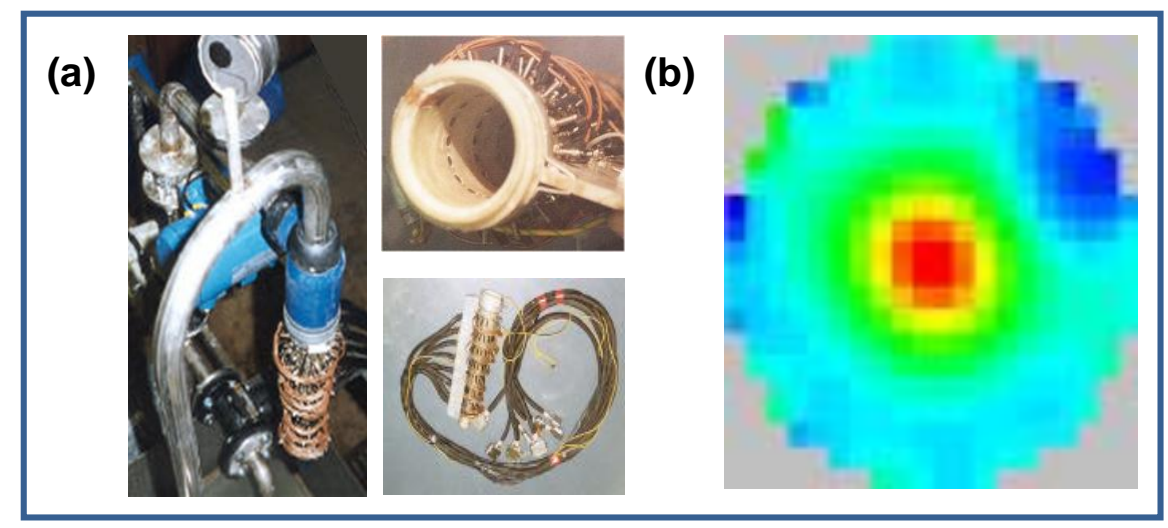

Figure 3-1: (a) Installation of ERT sensors to hydrocyclone system; (b) ERT image of dense flow separation (permission for reuse from Dyakowski et al., 2000, granted by Elsevier)

Results show that fault conditions, such as roping, spigot detachment and underflow blockage, could be identified from the resultant instantaneous conductivity images. Figure 3-1 (b) provides a typical ERT image obtained for dense flow separation conditions. These results suggest that the technique could be used for the determination of the operating state and detection of fault conditions.

Although the spatial resolution of images are low (as depicted in figure 3-1 (b)), it is deemed sufficient for this application. ERT yields further advantages as it is fairly robust (Dyakowski et al., 2000) and allows high speed imaging (between 100 and 200 frames per second). To interpret tomographic images at high speed rates, however, image reconstruction algorithms are required which are considered to be computationally complex (West et al., 2000). 


\section{(b) Electrical impedance tomography (EIT)}

EIT is used to map electrical impedivity or resistivity distributions. Williams et al. (1997) and Gutiérrez et al. (2000) report on a study that employed this technique to measure air core formation and density distribution inside a hydrocyclone.

The experimental setup consisted of 16 plate electrodes fitted externally to a hydrocyclone body. Images of the underflow discharge were also taken for the purpose of correlating with the EIT results.

Resistivity images illustrated the expected relations between solid concentration and air core development. As the solids content (and the sediment build-up) was increased, the air core decreased in size. Furthermore, resistivity images indicated that a fully developed air core corresponded with a spray-like discharge. An absent air core, on the other hand, was shown to correspond with rope-like discharge.

The results suggest that EIT can be used to identify the hydrocyclone operating state. Similar to ERT, low resolution images were generated while an imaging speed of more than 100 frames per second was achieved.

\section{(c) Electrical capacitance tomography (ECT)}

The application of ECT as a monitoring technique is briefly detailed by Williams, Dyakowski et al. (1995). This technique generates images of electrical permittivity distributions and in the case of Williams, Dyakowski et al. (1995), it was specifically applied to image the underflow discharge.

A monitoring setup, consisting of 12-electrode capacitance sensors, was used. These sensors were fixed in two vertical planes in the underflow region (represented as A-A and B-B in figure 3-2).

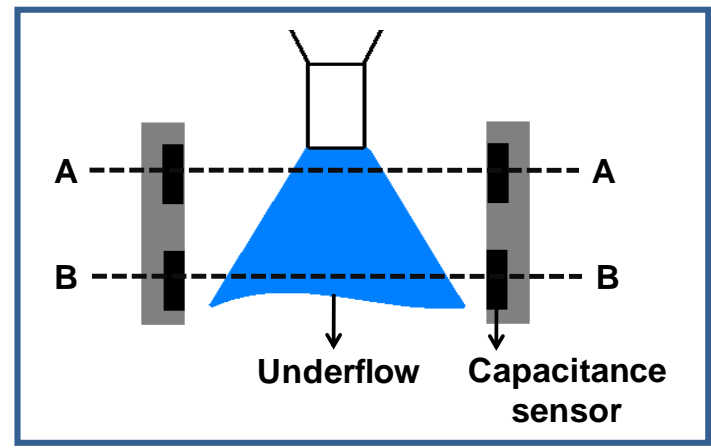

Figure 3-2: Electrical capacitance tomography applied to image hydrocyclone underflow (redrawn from Williams, Dyakowski et al., 1995) 
Results are presented as discharge profiles of different sizes and shapes. These profiles enable possible quantification of the discharge oscillations, since the images are produced at high speed (100 frames per second). Blocking, in particular, can also be readily detected from the profiles.

\section{(d) X-ray tomography}

Galvin and Smitham (1994) employed X-ray tomography to determine the density distribution in a hydrocyclone.

The monitoring setup used by Galvin and Smitham (1994) consisted of an X-ray plate, fastened to the back of a glass hydrocyclone. Scans of the X-ray film were made at three different locations to determine the particle density across the cyclone diameter. To improve image resolution, the hydrocyclone was operated as a dense-media cyclone (cyclones for which densities are manipulated by adding a medium to the feed). High density magnetite particles with strong $\mathrm{X}$-ray absorbance were therefore added to the system.

Although results show that the measured image density profiles agree very well with calculated values, the technique requires refinement. Areas of improvement include the calibration technique, increasing the signal-to-noise ratio and extending the study to components other than magnetite. An exposure time of 20 seconds was furthermore required, which measures relatively long in terms of sampling time.

\section{(e) Ultrasound tomography}

The work of Schlaberg et al. (2000) describes the implementation of an ultrasonic tomography system to measure the size and position of the air core. A hydrocyclone was modified by fitting 16 ultrasonic transducers externally to the device.

The results indicate that ultrasound tomography is a feasible and robust alternative to measure the air core size and position. Work performed by West et al. (2000) also state that the air core size measurements of ultrasound tomography are in close agreement to that obtained with ERT.

Images could be observed at a rate of six per second while a low image resolution of $50 \times 50$ pixels was achieved. Measurements could further be improved by fitting more transducers. However, the number of transducers is limited to the available space (size of hydrocyclone and transducers). In terms of industrial applicability, Schlaberg et al. (2000) furthermore considers the implementation to be very complex. 


\subsubsection{Acoustic monitoring}

\section{(a) Acoustic monitoring applied to upper hydrocyclone body}

Vibrations are inherent to a hydrocyclone's internal flow structure due to the presence of particle impact, highly turbulent vortex motion, air core instabilities and discharge oscillations (Neesse, Schneider et al., 2004; Neesse and Dueck, 2007; Sripriya et al., 2007). Hou et al. (2002) utilized this characteristic by measuring the acoustic emissions from a hydrocyclone's upper body and applying it as a monitoring technique. Similar work has previously been reported by Williams et al. (1996) and Hou et al. (1998).

In the Hou et al. study (2002), a 190-kHz piezoelectric sensor was mounted externally on the conical section of a hydrocyclone. Acoustic emissions were measured while altering the operating variables of the system.

It was found that the spectral characteristics of the signals are very sensitive to changes in the operating variables. Quantitative relationships between the operating variables and the spectral characteristics of the acoustic signals could be determined by use of multivariate statistical analysis. In general, these relationships predicted the operating variables with a high degree of accuracy.

Based on the fact that the signal spectral characteristics describe internal flow variations, it is envisaged that the technique can be used in future for the prediction of performance indicators and the transition in operating states. Furthermore, the sampling range $(0-1000$ $\mathrm{Hz}$ ) deems sufficient in terms of sensitivity and a satisfactory sampling rate was also realized (2000 readings per second). For industrial applications though, it is important to consider that experience is required for the correct placement of the sensors. Equipment such as pumps could contribute to the measured emissions and therefore determines the required sensor type and position (Williams et al., 1996).

\section{(b) Acoustic monitoring of underflow discharge}

Other work involving acoustic monitoring includes that of Neesse, Schneider et al. (2004), which focused on the underflow as the monitored feature. In order to measure the discharge oscillations, an acceleration sensor was mounted on the exterior of a hydrocyclone, near the spigot.

The resultant acoustic spectrum (shown in figure 3-3) indicates that rope-like discharge could be recognized as high amplitudes in the low frequency range, while the vibrations 
become damped at higher frequencies (100 to $600 \mathrm{~Hz})$. On the other hand, spray discharge shows significant vibrations at the higher frequencies.

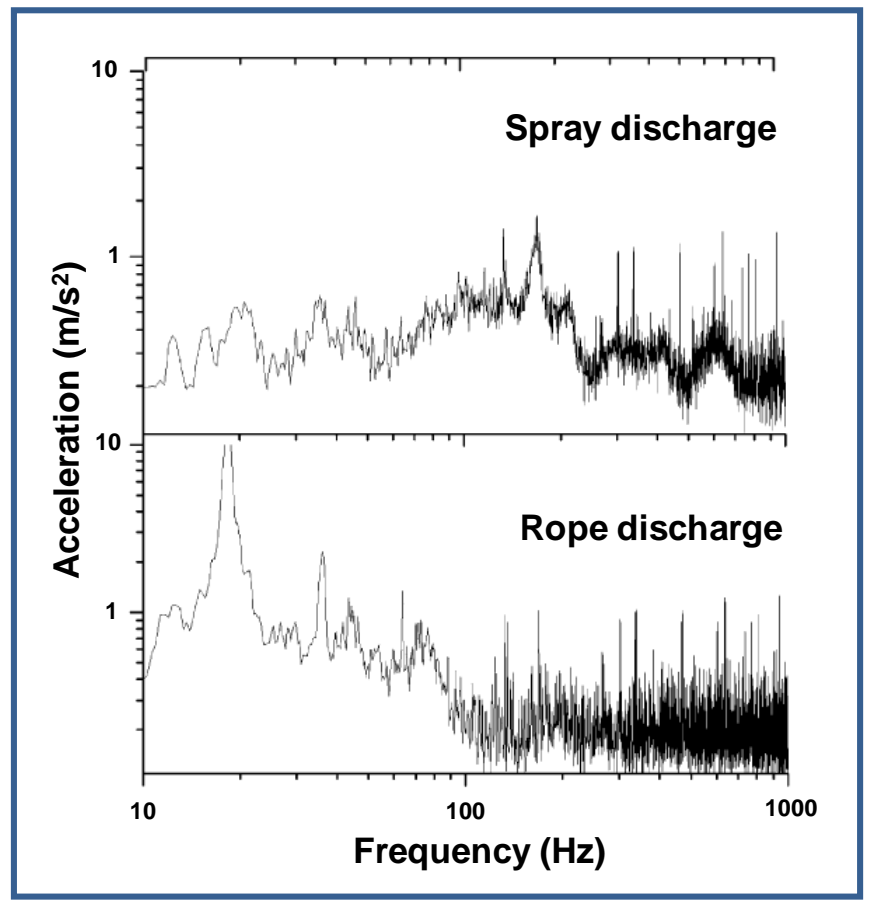

Figure 3-3: Acoustic spectrums of hydrocyclone for dilute and dense flow separation conditions (permission for reuse from Neesse, Schneider et al., 2004, granted by Elsevier)

Although the major operating states were identifiable from the acoustic spectrum, it was suggested that further investigations are necessary for improved understanding of underflow acoustic emissions. The suggestions included the development of a more robust setup.

\subsubsection{Mechanical detection}

A mechanical device used to measure the shape of the discharge stream was invented by Hulbert (1993). It consists of a pivoting arm mounted close to the spigot orifice. On the end of the arm is an abrasion resistant tip which is arranged in such a way that the tip rides on the edge of the conical discharge stream. The angle of the arm indicates the angle of the underflow, which is measured by an angle detector.

In 1986, the device was incorporated in a grinding circuit control system at Vaal Reefs Gold Mine (Mintek, 1992). Although the control system resulted in an increase of the grinding circuit efficiency, the angle detection device was found to be maintenance intensive (Hulbert, 2010). Since the device relies on contact with the underflow stream, it undergoes mechanical wear and requires regular replacement. 


\subsubsection{Gravimetric method}

Following on the work of Schweitzer (1972), Neesse, Schneider et al. (2004) used a weighing procedure to determine the amount of sediment within a hydrocyclone. For this monitoring system, the modifications required involve attaching the hydrocyclone to a load cell and fitting the inlet and overflow orifices with flexible connections. A schematic diagram of the described experimental setup is provided in figure 3-4.

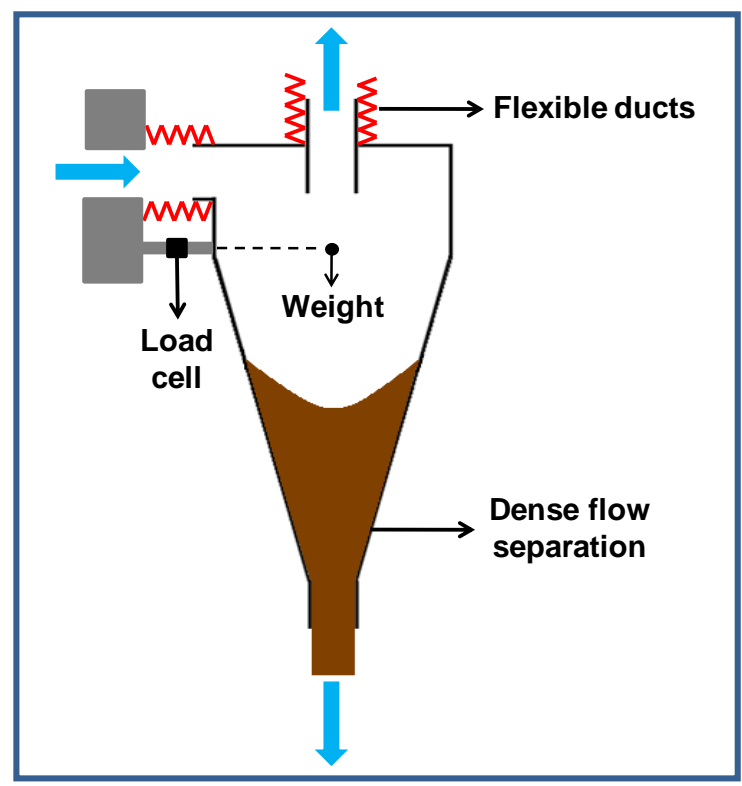

Figure 3-4: Determination of hydrocyclone weight by use of a weighing cell (redrawn from Neesse, Schneider et al., 2004)

From the results it was found that there exists a clear dependency of the measured hydrocyclone weight on the feed solids concentration. This method therefore allows a direct and fast way of determining the operating state. However, the technique is not robust since the flexible connections cause disturbing forces which superimpose the signals. Special software is required to compensate for these effects, rendering this method less practical.

\subsubsection{Ultrasound monitoring}

A very recent technique patented by Olson et al. (2006) entails the detection of the underflow shape by use of an ultrasonic sensor. For the monitoring system, the hydrocyclone is fitted with a splash skirt (a panel below the spigot used to reduce splashing), onto which an ultrasonic sensor is mounted. The peak frequency response of the sensor is $40 \mathrm{KHz}$.

Outputs from the sensor allow the identification of the various operating states by monitoring the amount of vibration. For dense flow separation, vibrations against the 
splash skirt are at a maximum. As the underflow discharge decreases in size, the amount of vibration also decreases. Based on this approach, the patent claims to successfully identify the various operating states as well as the onset of roping.

\subsubsection{Image analysis techniques}

\section{(a) Image analysis of air core}

Castro and Concha (1996) applied video image analysis to measure the air core diameter. In order to record the air core, a hydrocyclone was modified by creating an opening in the upper part of the body. A lighting system was furthermore installed to illuminate the air core. Experiments were carried out on a pilot plant facility.

Images revealed that the air core could clearly be distinguished from surrounding slurry. It could also be seen that the air core becomes very unstable during dense flow separation, disappearing completely at times. By incorporating air core diameter measurements, a semi-empirical model was set up for the specific pilot plant facility. The model proved useful for the prediction of dense flow separation in this regard.

\section{(b) Image analysis of underflow angle}

Another investigation into the use of image analysis as a monitoring technique was conducted by Petersen et al. (1996). In this case, the measured feature, the underflow shape, was monitored by determining the underflow angle.

The monitoring setup used for experiments consisted of a VHS-C (Video Home System Compact) video camera positioned to focus on the underflow stream. Tests were performed in both a laboratory and an operating plant. As detailed in a follow-up report of the study (Van Deventer et al., 2003), angle measurements were combined with fundamental, semiempirical and empirical models to develop a soft-sensor (virtual sensor which uses available data to make process predictions).

Although the technique did not explicitly focus on the identification of the operating states, it deemed promising since results showed that the underflow angle serves as a valuable indicator of operating characteristics and hydrocyclone performance. The author furthermore promoted the technique by stating that it complies with the requirements of a hydrocyclone monitoring system. However, due to computational limitations at the time, the video data could not be processed as a continuous data set. Discrete images were rather extracted and used to calculate an average underflow angle. 


\section{(c) Image analysis of underflow shape based on laser-optical detection}

Image analysis of the underflow discharge by means a laser-optical measuring device was investigated by Neesse, Schneider et al. (2004). Figure 3-5 illustrates the setup of the monitoring system which consisted of laser beam directed towards the underflow. A CCD (Charged Coupled Device) video camera was used to record the refracted image of the beam.

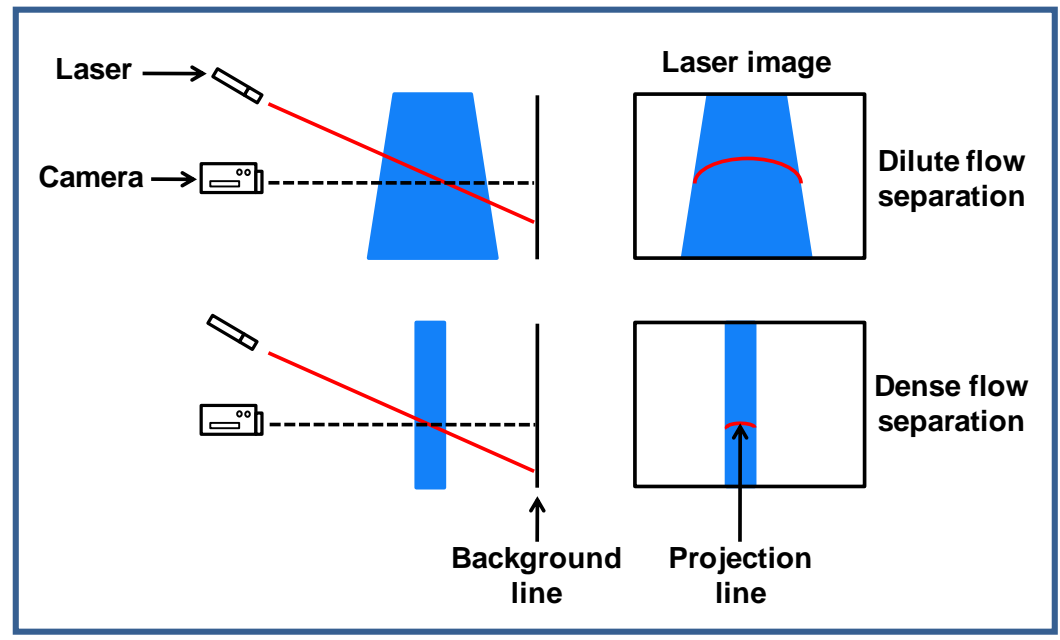

Figure 3-5: Monitoring setup of laser-optical detection method (redrawn from Neesse, Schneider et al., 2004)

In the resultant images both the refracted laser image and the distance to the background line were classified as indicators of the operating state. Preliminary results indicated that dilute and dense flow separation could be identified.

In a comparative study of various types of monitoring techniques, Neesse, Schneider et al. (2004) considered this technique the most promising based on the mentioned findings, the non-intrusiveness of the setup and the low cost equipment.

\subsection{Motivation for image analysis as proposed monitoring technique}

Based on the potential to identify operating states, all the tomographic techniques seem promising. This is also true when considering the sufficiently fast sampling times (with the exception of X-ray tomography). Wide spread adoption of these techniques, however, are restricted due to the complex image reconstruction algorithms required for on-line image interpretation (West et al., 2000). Some of the tomographic techniques, such as ERT, furthermore require extensive modifications to the hydrocyclone setup. 
Acoustic monitoring appears advantageous in terms of the simple setup and achieved sampling times. With regards to the identification of operating states, both mentioned applications are, however, subject to further investigation. The robustness of acoustic monitoring is also subject to correct placement of the sensor, which requires expert knowledge.

The mechanical detection method developed by Hulbert (1993) is a simple method to monitor the underflow angle. Although positive results have also been obtained from the technique's industrial application, its continuous use is limited since it is not a contact-free method. In other words, the setup is maintenance intensive and requires regular replacement (Hulbert, 2010).

Even though the gravimetric method yields positive results, this technique is also disregarded due to its lack in robustness. The monitoring setup furthermore requires extensive modifications to existing hydrocyclone setups.

Ultrasound monitoring of the underflow discharge produces positive results in terms of identification of operating states. The monitoring system furthermore consists of a simple setup, data interpretation technique and achieves a sufficiently fast sampling time. For these reasons, the ultrasound monitoring of the underflow discharge is considered a feasible monitoring option.

Although image analysis of the air core can be used to identify the hydrocyclone operating state, it is not considered a practical option since the technique requires the hydrocyclone body to be altered significantly. Image analysis of the underflow discharge, however, is deemed more advantageous. The preliminary work of Petersen (1996) suggested the potential of the technique, while the recent work performed by Neesse, Schneider et al. (2004) focused on the development of a viable monitoring system. In the latter study, the monitoring system involved a simple setup and was found to fulfil the requirements of a hydrocyclone monitoring system. Preliminary results also indicated that the operating states could be identified successfully.

Ultrasound monitoring and image analysis of the underflow discharge seem comparable in terms of potential application as hydrocyclone monitoring techniques. However, image analysis of the underflow discharge stream is favoured, since it also enables continuous visual observation of the underflow discharge stream and spigot region. If a fault condition is picked up by the monitoring system, troubleshooting might also be simplified through investigation of these readily accessible visuals. The fact that whole images are obtained as output, also allows the extraction of a variety of data sets. For example, the underflow shape or change in motion of the underflow discharge might be extracted. Opportunities also exist to extract data such as particle size distributions from such images. It is further 
theorized that a very simple image analysis technique can be used to extract data able to indicate the operating state. From the findings of this evaluation, image analysis of the underflow is considered a promising alternative for the on-line monitoring of a hydrocyclone and is therefore the subject of this investigation.

\subsection{Proposed entity for quantification of variation in underflow shape}

As mentioned previously, Petersen et al. (1996) and Neesse, Schneider et al. (2004) monitored two different entities to quantify the underflow shape: Petersen (1996) used video imaging to monitor the underflow angle, while Neesse, Schneider et al. (2004) monitored the projection line of a refracted laser through the underflow stream. The underflow width is proposed as an alternative for the quantification of the underflow shape. Since the underflow width is essentially a straight line measurement, it is expected that the determination and analysis thereof will rely on simpler image analysis techniques, than in the latter two cases.

\section{CHAPTER SUMMARY}

In terms of sensing capabilities, the following aspects should be considered for a hydrocyclone monitoring system: sensitivity, non-intrusiveness, robustness, sampling times smaller than $1 \mathrm{~s}$ and low cost equipment. It is furthermore preferred that the system should be simple to use, require little modification to the current setup and involve little maintenance or replacement. For most monitoring techniques investigated in the past, compliance with these requirements is unsatisfactory and the techniques have therefore not found broad adoption yet. For example, tomographic techniques are considered too complex and require extensive modification to hydrocyclone setups. Acoustic monitoring necessitates refinement and expert knowledge before practical implementation can be considered. The mechanical detection method is maintenance intensive, while the gravimetric method is not robust. Ultrasound monitoring and image analysis of the underflow does, however, prove advantageous in terms of the hydrocyclone monitoring requirements. Image analysis is promoted though, since it also enables continuous visual observation of the underflow discharge. The proposed entity for monitoring underflow shape variation is the underflow width, since it is expected that this would involve simple image analysis techniques. 


\section{CHAPTER 4: EXPERIMISTTAL MIETHODOLOGY}

Chapter 4 details the implementation of the image analysis monitoring system on an experimental hydrocyclone setup. This includes a description of the equipment setup, the monitoring system specifications and the developed experimental procedure. The performed experiments are presented as six different case studies.

\subsection{Experimental setup}

Figure 4-1 provides an illustration of the general hydrocyclone setup used for data collection.

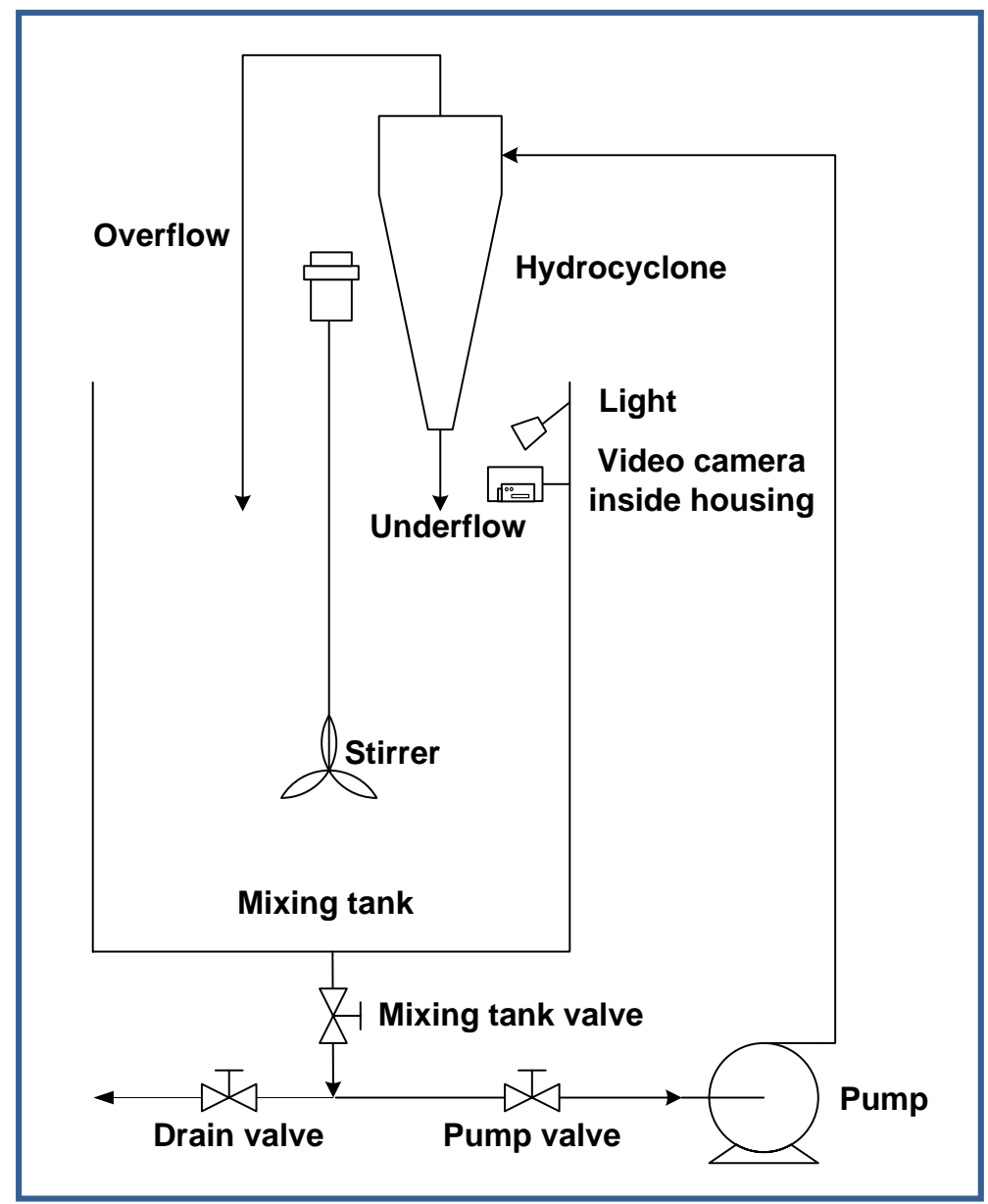

Figure 4-1: Schematic of general hydrocyclone setup

The setup consisted of a hydrocyclone suspended above a mixing tank. Both the underflow and overflow streams discharged into the mixing tank. These two streams were subsequently mixed by a stirrer and then fed back to the hydrocyclone inlet through the use of a fixed speed centrifugal pump. As result, a basic recirculation system was formed. To 
give an indication of the size range of equipment, equipment specifications for one setup are provided in appendix B-2.

To capture video data of the underflow discharge, a video camera was positioned directly across the spigot. This was done by placing the video camera in a housing mounted on the side of the mixing tank. Videos were recorded through a sightglass on the front panel of the housing. The housing was mainly used to protect the video camera from spluttering droplets. For cases where additional lighting was used, a light was mounted on the side of the mixing tank and directed towards the underflow stream.

\subsection{Experimental procedure}

The experimental procedure that was followed consists of four main steps. These steps are briefly summarized in the following section while a detailed procedure is provided in appendix B-1. Risks and safety aspects associated with the experimental work are furthermore detailed in appendix B-3.

\subsubsection{Preparation}

System preparation involved positioning the video camera and light by directing it towards the spigot and underflow region. A representative bulk ore sample was also used to determine the typical particle size distribution of the ore.

\subsubsection{Start-up procedure}

This step involved the activation of the system and the video camera. To enable flow recirculation, the mixing tank was partially filled and the stirrer and pump switched on. This pure water flow system then served as initialization point for all experiments by representing the start of dilute flow separation.

\subsubsection{Operating procedure}

To simulate varying feed concentrations, small amounts of ore were gradually added to the feed stream. The sightglass was wiped clean to remove any dirt caused by ore addition and droplets produced by spluttering. After each ore addition, five minutes were allowed for the system to reach steady state before the next ore addition. As the amount of ore in the system progressively increased (and feed stream concentration increased), changes in the operating state were induced. Consequently the operating state varied from dilute flow separation, to transition state and finally to dense flow separation as illustrated in figure 42. The concentration of ore in the system was monitored manually, as detailed in appendix B-5. 


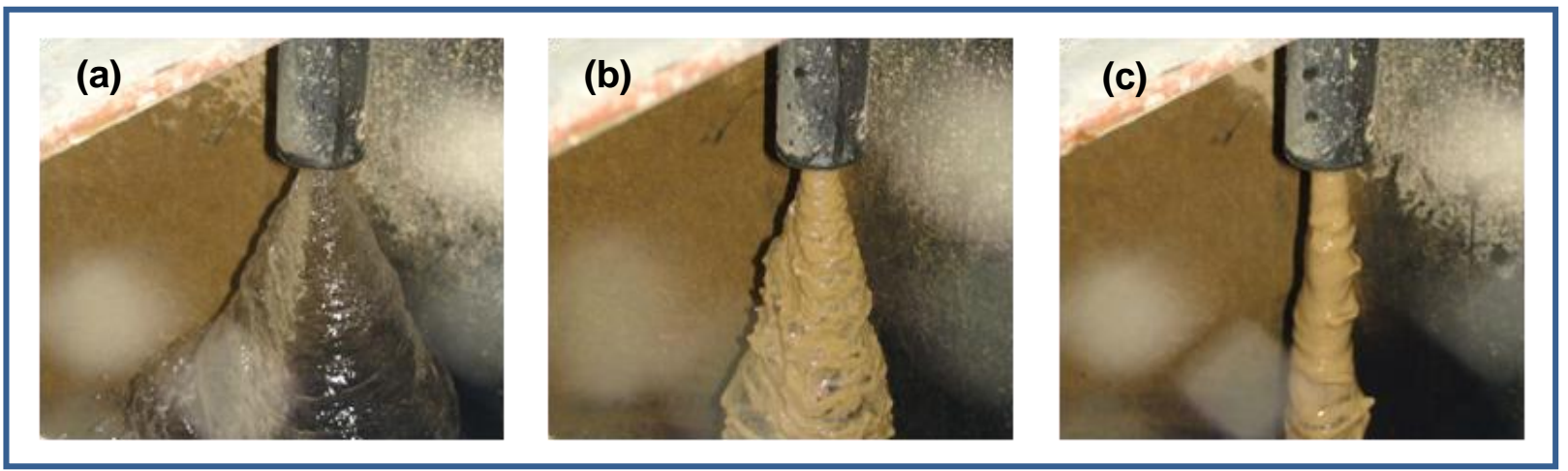

Figure 4-2: Variation in operating state from (a) dilute flow separation, to (b) transition state and finally (c) dense flow separation

\subsubsection{Shut down procedure}

Upon recognition of rope-like discharge, the operating state was characterized as dense flow operation. The system was again allowed to achieve steady state. At this point, no more ore was added to the system to prevent the hydrocyclone, pump and pipelines from blocking. Subsequently, the video camera was deactivated. Hereafter, the system was drained and flushed of ore.

\subsection{Specifications of monitoring system}

In the previous studies incorporating image analysis, the authors stated that the technique demonstrated compliance with the hydrocyclone monitoring requirements (Petersen et al., 1996; Neesse, Schneider et al., 2004). To ensure that this would also be true for the current study, monitoring equipment was acquired that would potentially meet these requirements. The following section therefore details the specifications of the monitoring equipment related to the hydrocyclone monitoring requirements.

A Sony video camera, model DCR-SX44E, was used to record the underflow stream during experiments. The sensor used to convert light to electrical signals is of the CCD (Charged Coupled Device) type.

\subsubsection{Sensitivity}

Video camera sensitivity is generally specified in terms of resolution and light sensitivity (Mesnik, 2004). The utilized video camera has an image resolution of $480 \times 720$ pixels and light sensitivity of 3 lux (measure of luminance) respectively. A true assessment of whether the resolution and light sensitivity, together with the developed image analysis technique, are sensitive enough to measure variation in underflow width effectively, can only be made 
upon interpretation of the results. This matter will therefore be readdressed in subsequent discussions.

\subsubsection{Non-invasive sensing}

A camera lens with a $60 \times$ optical zoom capability, allows the video camera to be situated some distance from the underflow width. Due to the structural layout of the hydrocyclone systems, the camera position was limited to a region between $0.3 \mathrm{~m}$ and $0.4 \mathrm{~m}$ from the underflow stream. This distance however, is still large enough to ensure a completely noninvasive sensing technique.

\subsubsection{On-line sensing with sampling time $<1$ s}

The sampling time of the video camera is related to its shutter speed. The camera used records videos at a satisfactory speed of 25 frames per second.

\subsubsection{Robust sensors (insensitive to blinding and mechanical stress)}

Since the camera is mounted in a housing and positioned on the side of the mixing tank, it is not subjected to any mechanical stress. Spluttering droplets that attach to the sightglass, could however, possibly blind the camera. The extent to which this problem affects the robustness is assessed in chapter 6 .

\subsubsection{Cost-effective instrumentation}

The Sony DCR-SX44E was relatively inexpensive. Other costs involved with the addition of the image analysis monitoring system included minor expenses such as the video camera housing and light source.

\subsection{Performed case studies}

Various ore types were incorporated in the case studies to investigate the applicability of the monitoring system to different ore material. These ore types included gold, ilmenite and PGM (platinum group metals) ore from both Platreef and the Merensky reef. Variation in ore material furthermore introduced different ore properties such as density, particle size distribution and colour. Another aspect that varied for some of the case studies was the use of lighting. The different case studies are presented in table 4-1 and subsequently discussed with regards to any differences or similarities. 
Table 4-1: Specifications of performed case studies

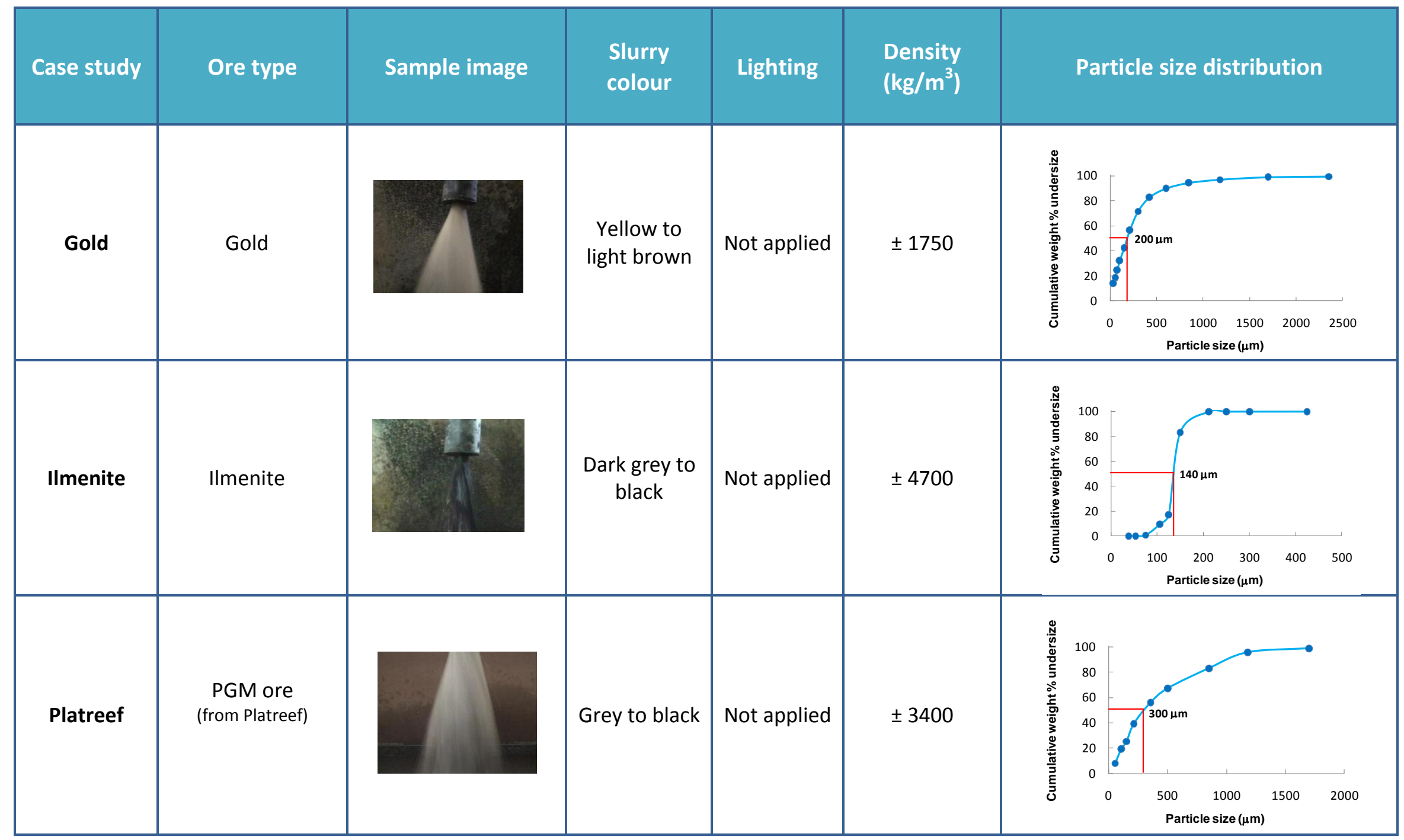


Table 4-1 (continued): Specifications of performed case studies

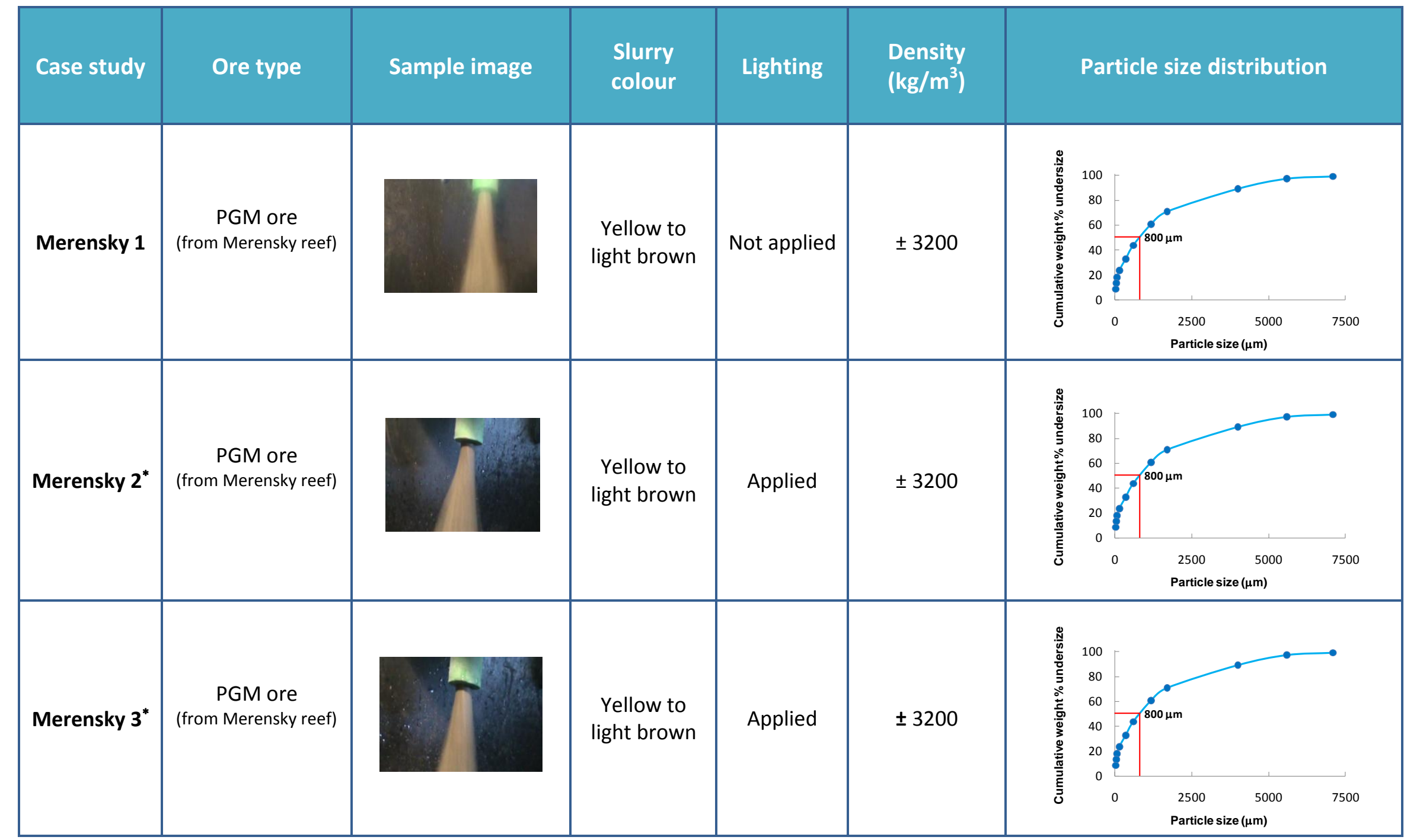

*Merensky 2 and 3 case studies are considered duplicates since the same video camera position and ore were used. 


\subsubsection{Slurry colour}

Due to the different ore types used, variations in ore and slurry colour were experienced (as illustrated in the sample images of table 4-1). These colour differences are mentioned since the effect thereof is investigated in the results section.

\subsubsection{Application of lighting}

After performing the first four case studies, it was expected that lighting might be used to accentuate the underflow stream and thereby simplify image analysis. This speculation was researched by incorporating the use of lighting in the last two case studies (Merensky 2 and 3).

\subsubsection{Ore densities and PSD's}

Table 4-1 shows that ilmenite was the densest ore used, while gold was the least dense. In terms of PSD's, the Merensky ore spanned across the largest range of particle sizes. On the contrary, the PSD of ilmenite ore was restricted to smaller range in particle size. The indicated median sizes (the mid-point of the PSD's) furthermore give an indication of the range of particle sizes for each case study. Ultimately, it can be seen that a variety of densities and PSD's were incorporated by the use of different ore types. Sieve analysis results used to determine the PSD's of the different ore types are provided in appendix B-4.

\section{CHAPTER SUMMARY}

Experiments were performed on a basic hydrocyclone recirculation system, equipped with a video camera directed towards the underflow. All experiments were initialized at dilute flow separation conditions. Variations in operating states were simulated by gradually increasing the amount of ore in the system. The camera used complies with the hydrocyclone sensing requirements in terms of non-invasiveness, sampling time and cost. Assessment of the technique's sensitivity and robustness however, is dependent on the outcome of the image analysis results. Six case studies were performed with different types of ore: gold, ilmenite, PGM from the Platreef and PGM from the Merensky reef. Variations in the case studies include differences in ore colour, density, PSD as well as the application of lighting. 


\section{CHAPTझR 5: IMAGE ANALYSIS AND DIAGNOSTIC MONITORING}

The developed image analysis and supporting diagnostic monitoring techniques are presented in the following chapter. Firstly, image noise and its impact on the development of the image analysis technique is explained. Thereafter the various steps of both the image analysis and diagnostic monitoring techniques are detailed and motivated, where necessary. Finally, an overview of the conceptual calibration and on-line monitoring algorithms is provided.

\subsection{Image noise}

Noise, in context of this study, does not refer to audible noise but rather to undesired features in images. Since the presence of noise in images influenced the development of the image analysis technique, the types of noise are described before providing the details of the technique. The three types of noise that are distinguishable in images are background-, foreground- and random noise. Typical examples of these types of noise are provided in figure 5-1. Regions with prominent background- and foreground noise are highlighted in red.

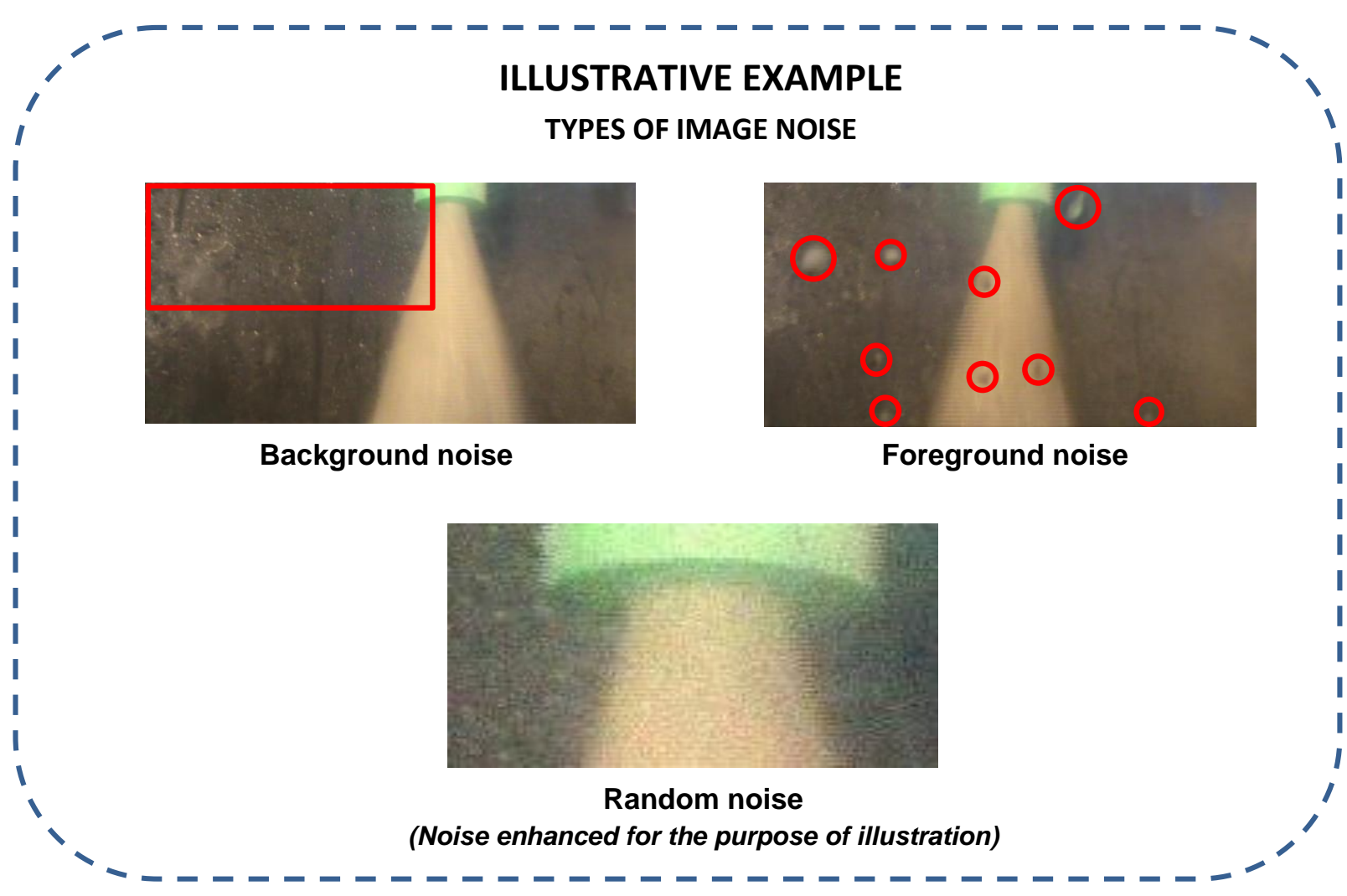

Figure 5-1: Types of noise in hydrocyclone video images 


\subsubsection{Background noise}

Spluttering droplets are an apparent feature in the hydrocyclone environment. The droplets are typically introduced to the background of images by collecting against the side of the mixing tanks. These droplets, especially in the close vicinity of the underflow, are treated as noise since their colour intensities closely resemble that of the underflow, hence reducing the interpretability to the computing system. Features with these characteristics could erroneously be recognised as part of the underflow.

\subsubsection{Foreground noise}

In certain cases, spluttering droplets also obstruct the foreground of the underflow image. This happens when droplets adhere to the sightglass of the video camera housing or cause streaks along the same surface. As with background noise, foreground noise could also be mistaken as part of the underflow.

\subsubsection{Random noise}

Random noise is a type of image noise which is inherent to all images, since it is produced by the camera itself. The extent of noise development is subject to the light sensitivity and exposure time of the sensor. This type of noise presents as small regions with colours and intensities different to that of the actual image (McHugh, 2010). Although random noise is visually less discernable than the other types of noise, the presence thereof complicates subsequent image analysis (as explained in section 5.2.7).

\subsection{Image analysis technique}

Two options were considered as feature extraction technique for the determination of the underflow width: edge detection and motion detection. In general though, edge detection is considered a non-trivial task, especially concerning the selection of an appropriate algorithm and the accompanying parameters (Acton and Mukherjee, 2000). In contradiction, motion detection can be executed as a very simple mathematical operation. Based on the simplicity of the method, motion detection was selected as the feature extraction technique.

An image analysis technique was consequently developed that primarily consists of motion detection, various noise reduction methods and supporting processing actions. In the next few sections, the various steps of the technique are described and motivated. Although each step was executed numerically, sample images from the Merensky 1 case study are provided to illustrate how the video data was influenced visually. 


\subsubsection{Image acquisition}

Footage recorded by the CCD camera was saved as separate video segments ${ }^{*}$ in MPEG format and subsequently imported into MATLAB ${ }^{T M}$. To perform image analysis, additional MATLAB $^{\text {TM }}$ toolboxes, the Image Acquisition and Image Processing Toolboxes, were also incorporated. The latter toolbox was used to divide video segments into individual frames denoted as $F_{k}$, where $k=1,2, \ldots N$. Based on the shutter speed of the camera, 30 frames were obtained for every second of video data. Further analyses, as described in sections 5.2.2 to 5.2.5, where performed on each individual frame or image, $F_{k}$.

\subsubsection{Conversion to greyscale colour field}

Images represented in colour require a lot of storage space since three colour values are assigned to each pixel (red, green and blue in the RGB colour field). The range for each colour value spans from 0 to 255 . To reduce the processing time and storage space required, the number of colours was therefore reduced by converting each image to the greyscale colour field. Essentially, the image was reduced from a three-dimensional to a one-dimensional array. Figure 5-2 provides an example of this colour conversion for the Merensky 1 case study.

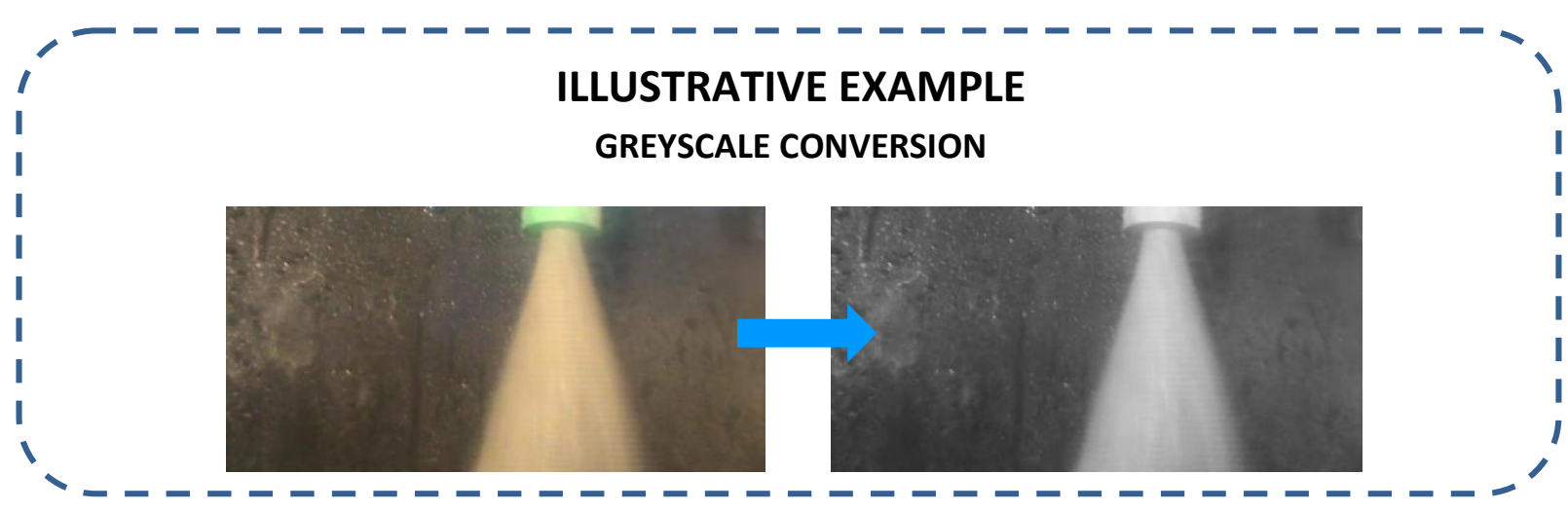

Figure 5-2: Image conversion from RGB to greyscale colour field

The conversion was numerically executed by applying equation 5-1 (default colour weights provided by MATLAB ${ }^{\mathrm{TM}}$ ).

$G S=0.2989 R+0.5870 G+0.1140 B$

Equation 5-1

where

$G S=$ greyscale pixel value,

$R=$ original red colour pixel value,

$G=$ original green colour pixel value and

\footnotetext{
* In the experimental video footage, underflow visibility was intermittently obstructed by ore additions and cleaning of the sightglass. The footage was therefore cut into separate segments, by removing sections where obstructions occurred. A standard video editing programme was used for this task. The video segments were analyzed individually and the outputs compiled at the end.
} 
$B=$ original blue colour pixel value.

As result, each pixel attained only one colour value between 0 and 255. Black is represented by 0 on one end of the scale while 255 represents white on the other end (Russ, 1995).

\subsubsection{Image enhancement through contrast and brightness adjustments}

Image enhancement refers to the improvement of image interpretability for both human observers and computing systems (Maini and Aggarwal, 2010). In context of this study, image enhancement refers to the methods used to make the underflow stream more recognisable to the computing system. The extent to which the underflow can be distinguished from surroundings is impeded by noise. Image enhancement was consequently used to reduce the visibility of background-and random noise to the computing system.

Of all the available image enhancement techniques, contrast and brightness manipulation (also referred to as intensity transformations) are considered to be some of the most straightforward techniques (Gonzalez and Woods, 2008). These techniques are based on the transformation of input pixel values to a desired output range. In this light, contrast adjustment was used to zoom in on a certain range of intensity values while brightness adjustment was used to centre the zoomed section on the values of interest (Smith, 1997).

A MATLAB ${ }^{T M}$ graphical user interface (GUI), Adjust Contrast Tool, was initially used to investigate the extent of contrast and brightness adjustments required. The settings that were established through this process were then specified as user inputs to the image analysis technique. These settings, which include the minimum, maximum and centre values of the zoomed section, were used to create an intensity transformation function, as illustrated in figure 5-3. To clarify this concept further, a sample calculation is provided in appendix C-1.

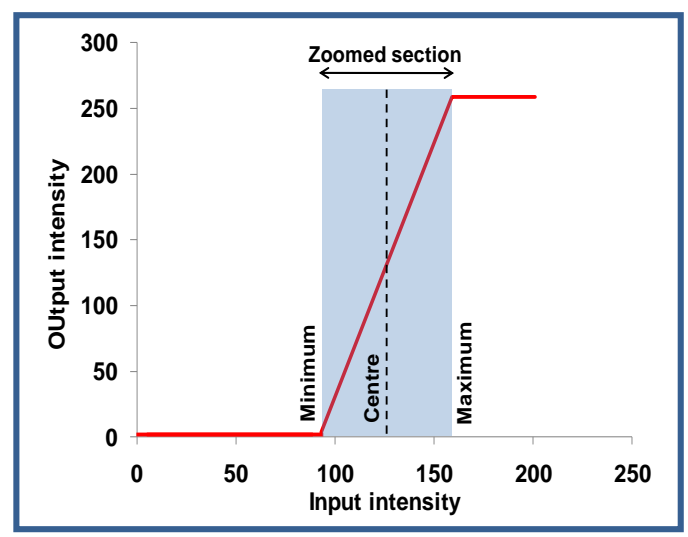

Figure 5-3: Intensity transformation function used for contrast and brightness adjustments 
Figure 5-4 shows how contrast and brightness adjustments were applied to achieve a more prominent underflow stream and fainter background noise for the Merensky 1 case study. Regions indicated in red rectangles highlight the most noticeable background noise reduction. The effect of image enhancement on random noise is not depicted in these images though, but is visually illustrated in a subsequent section (refer to section 5.2.7).

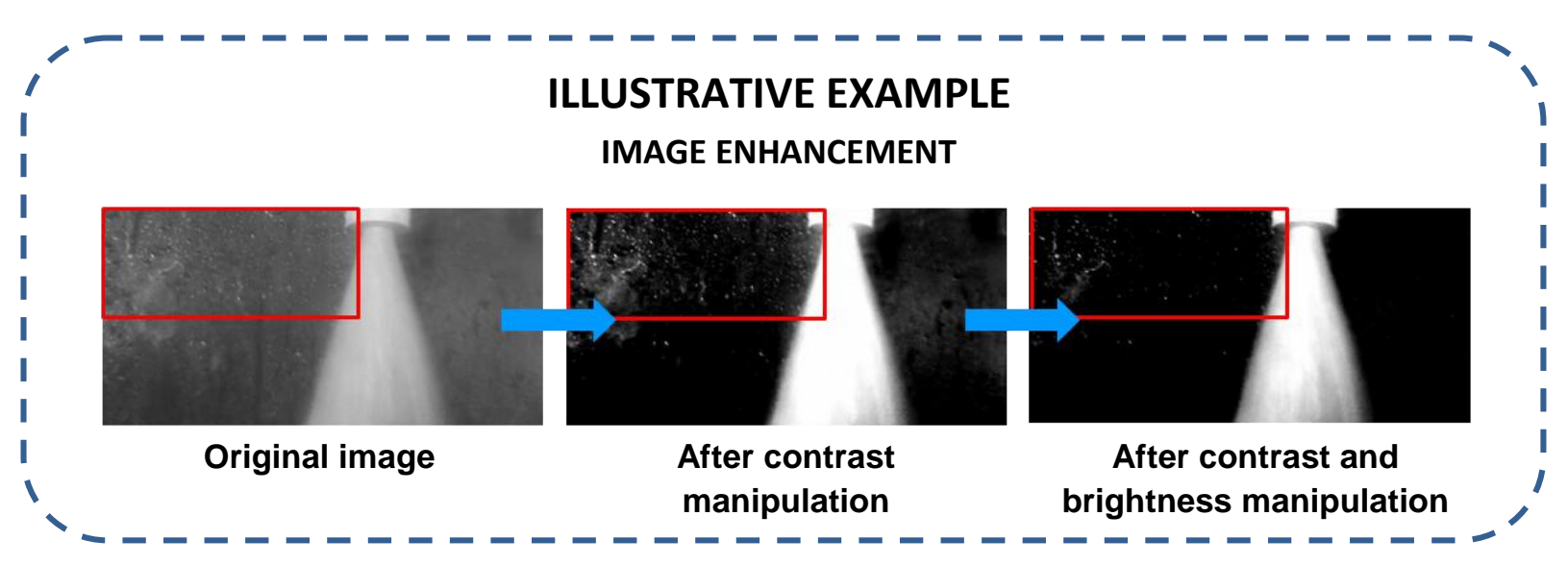

Figure 5-4: Effect of contrast and brightness manipulation on an underflow image

\subsubsection{Extraction of horizontal line}

To keep processing requirements to a minimum, a single horizontal line was extracted along which the underflow width was determined. The conceptual spray profile (refer to figure 55), as defined by Dombrowski and Hasson (1969), was considered for the specification of the line position.

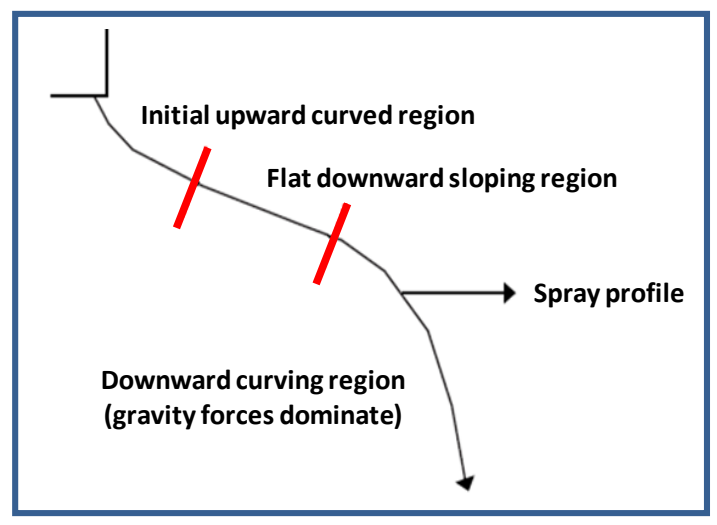

Figure 5-5: Conceptual spray profile as defined by Dombrowski and Hasson (1969)

If the line position is taken too close to the spigot opening, it will fall inside the initial upward curved region. The width variation in this region is minimal and would complicate the image analysis significantly. On the other hand, analysis at a point too far below the spigot opening might fall in a region where gravity forces are dominant, resulting in a downward turn of the discharge shape. To exclude the regions of insufficient variation and 
dominant gravitational effects, the line position was defined in the middle or flat downward sloping region. Ultimately, the line position could be chosen arbitrarily in this region, given the position remained the same for an entire a case study.

Initial assessment of the appropriate line position was performed with another MATLAB ${ }^{T M}$ GUI, namely, Image Tool. As example, the application of the conceptual spray profile for the Merensky 1 case study is illustrated in figure 5-6. The selected line position was matched to a $y$-coordinate, based on the image coordinate system of MATLAB ${ }^{T M}$. In this case the resultant line position is $y=190$.

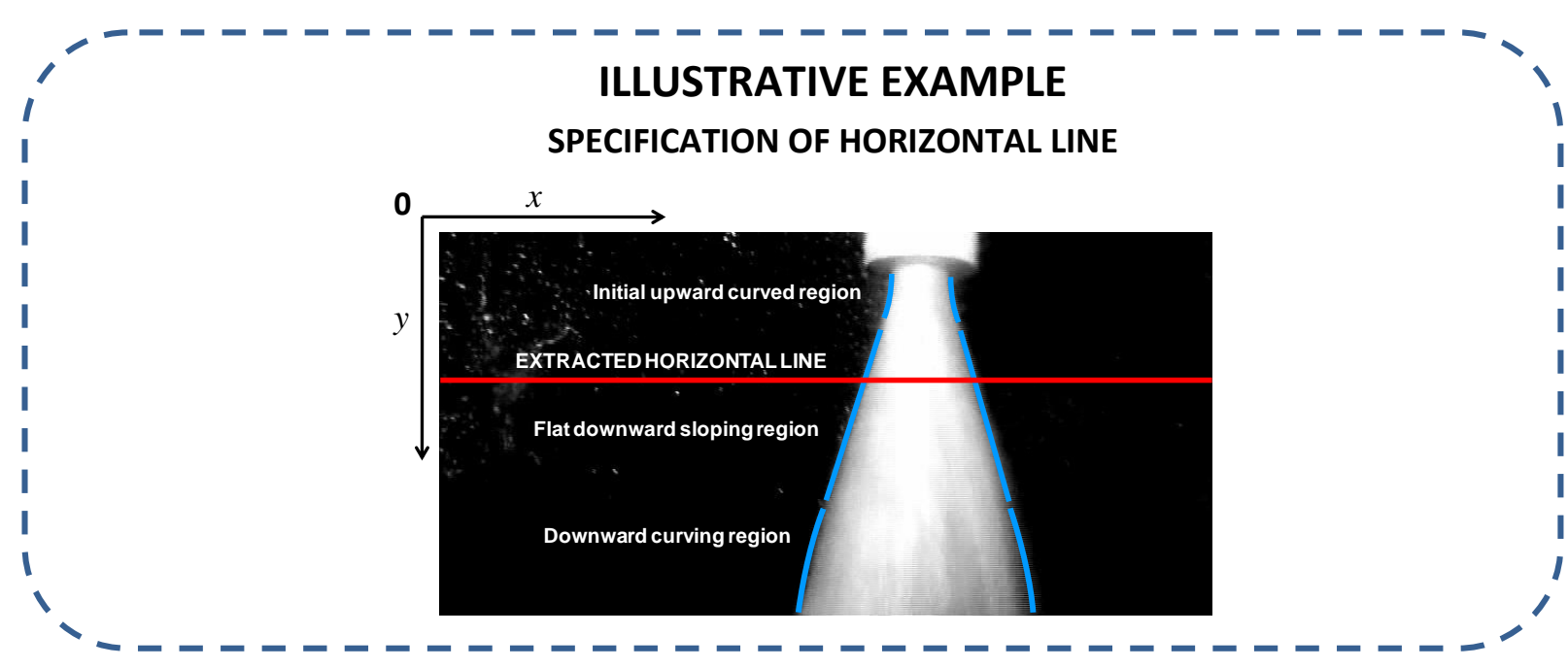

Figure 5-6: Specification of horizontal line for determination of underflow width

\subsubsection{Generation of intensity spectrum}

The extracted horizontal line was consequently used to generate an intensity spectrum for each frame. Generally, the underflow stream presents as a prominent intensity peak in this spectrum, as shown in figure 5-7. At this stage, there might be remaining background noise which will manifest in the spectrum, as shown in the provided example.

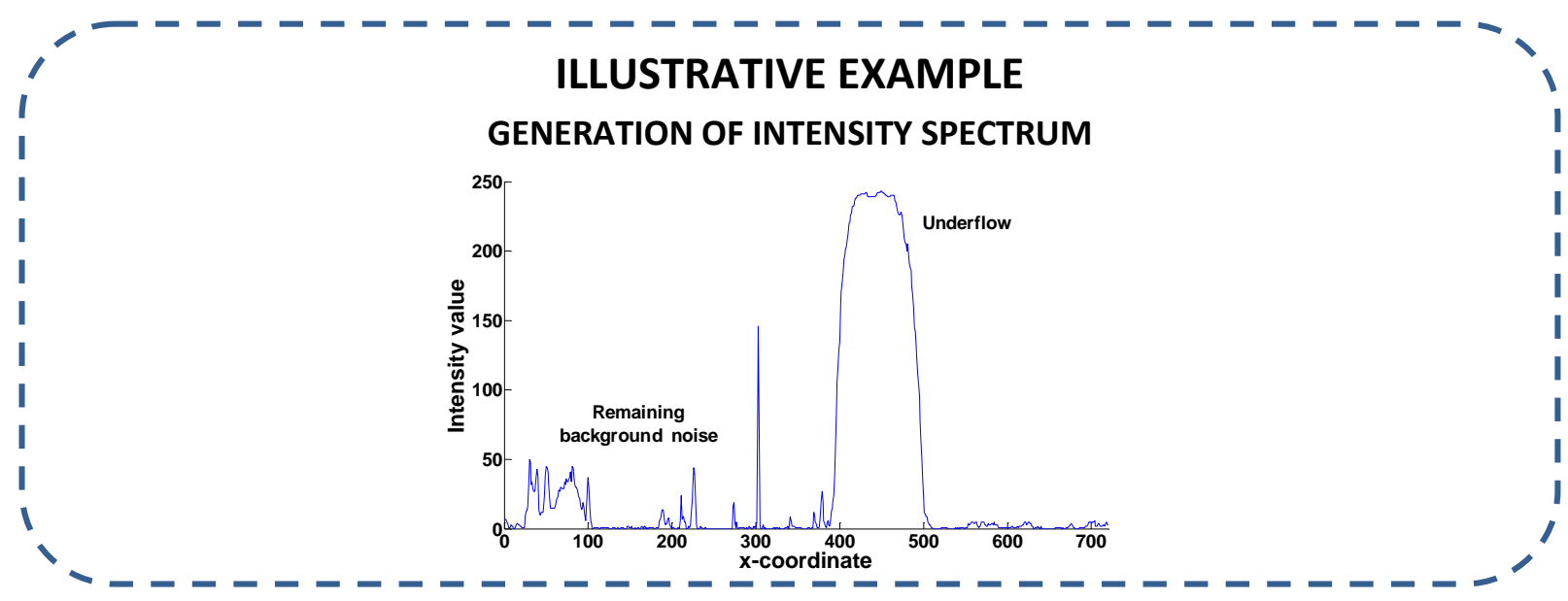

Figure 5-7: Example of intensity spectrum for Merensky 1 case study 


\subsubsection{Detection of interval search limits (ISL)}

To remove foreground noise and remaining background noise that could interfere with subsequent underflow recognition, ISL were determined. Figure 5-8 depicts the process used to determine the exact position of the limits for a segment of the Merensky 1 case study.

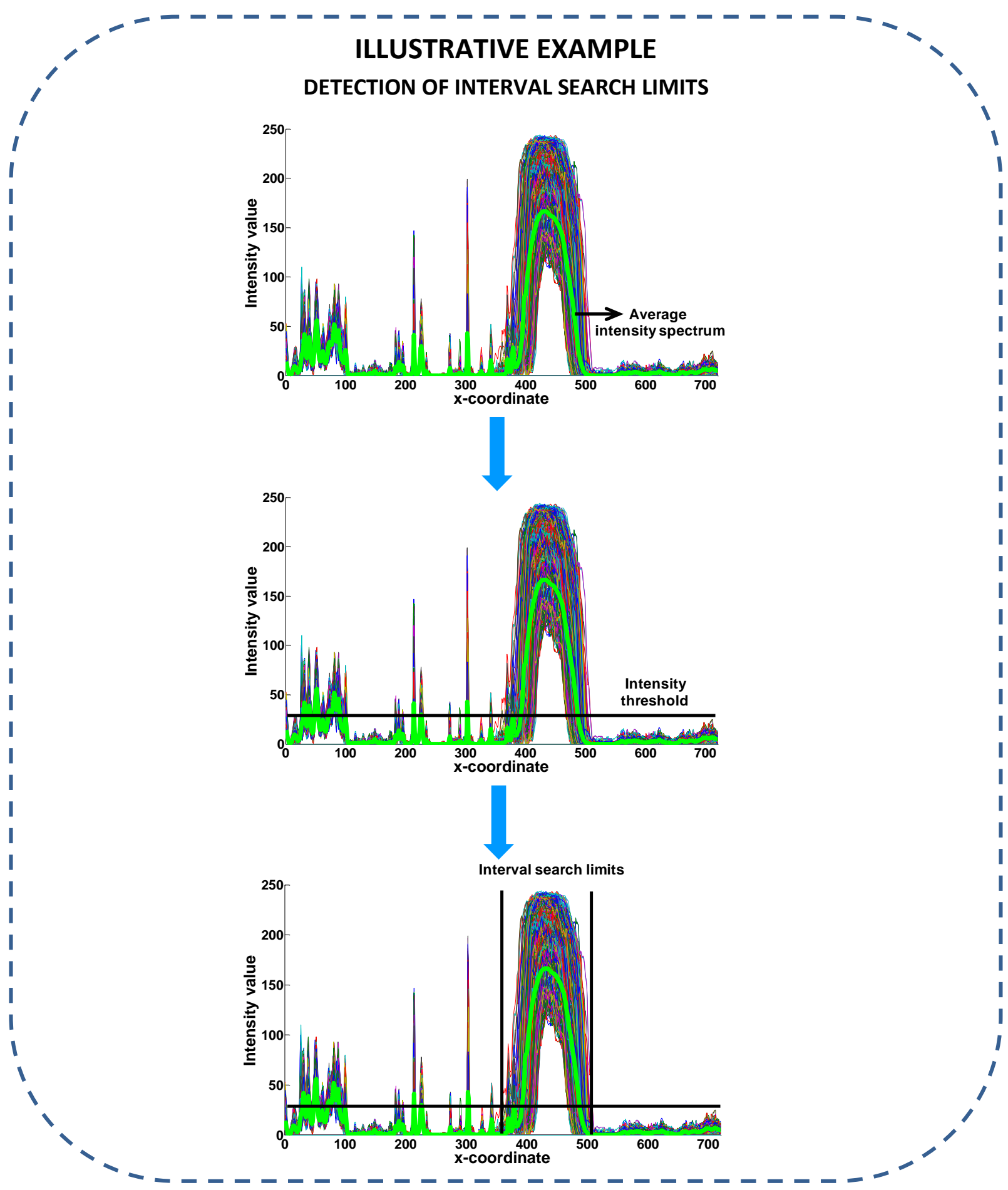

Figure 5-8: Detection of ISL based on compiled intensity spectra 
The determination of these limits was based on a compilation of the intensity spectra of 1800 frames (corresponding to one minute of video data). From this, an average intensity spectrum for the entire 1800 frames was calculated. This average intensity spectrum was used to calculate an average intensity value which was consequently used as a threshold limit. The largest region, with intensities above the threshold limit, was recognized as the underflow stream. The outer limits of this region were detected and subsequently dubbed the ISL. The position of the ISL were updated every 1800 frames.

A question might arise as to why ISL were not calculated for individual frames and thereby used to determine the underflow width? Figure 5-9 presents the answer to this question.

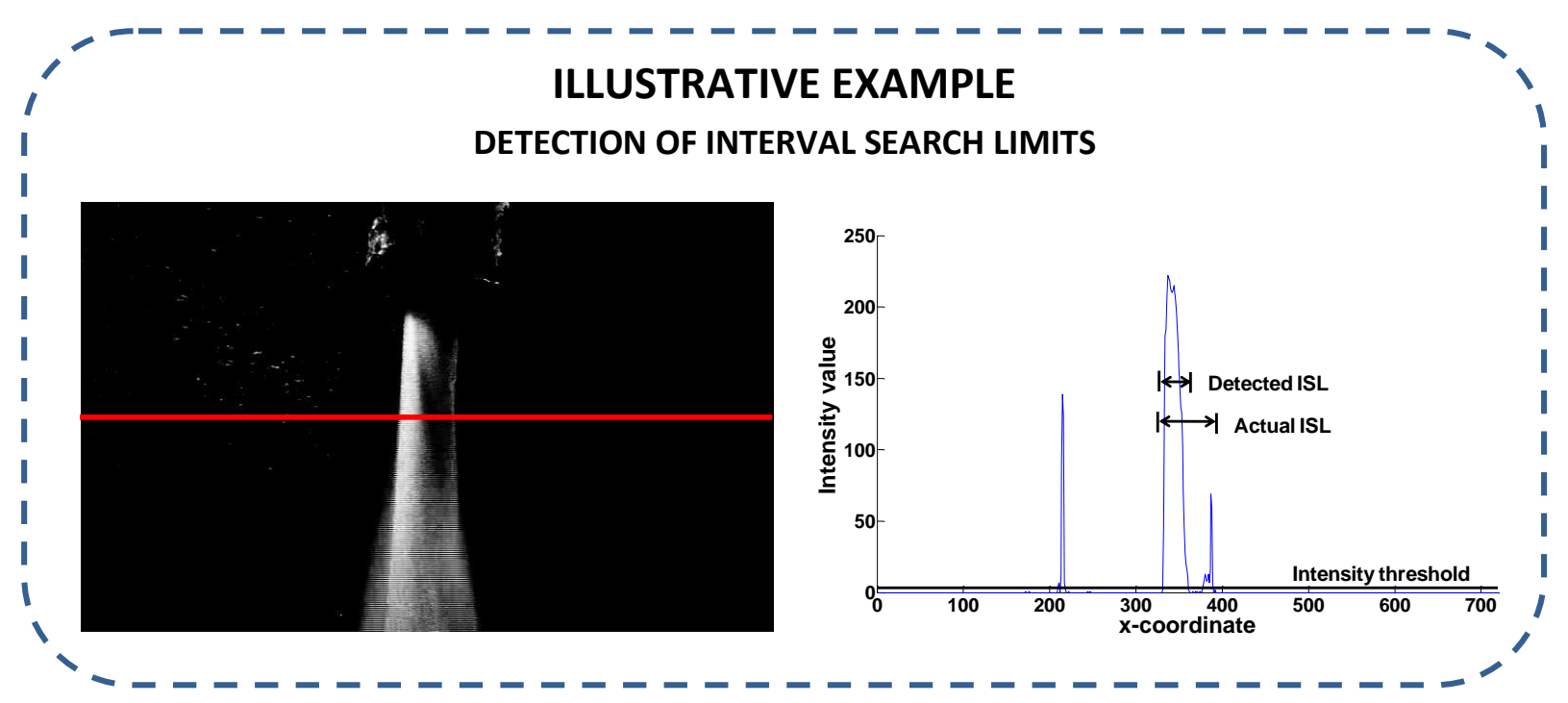

Figure 5-9: Underflow image and intensity spectrum from Gold case study depicting intensity discontinuity

Figure 5-9, from the Gold case study, illustrates an extreme discontinuity in the underflow intensity along the extracted horizontal line. In this case, slight discontinuity was introduced by the spigot that cast a shadow on the underflow stream. During the transition state, such discontinuities became prominent at intermittent moments when the underflow switched between spray and rope discharge. Similar discontinuities were witnessed for all other case studies.

If the ISL for this particular frame were calculated, the resultant limits would understate the true underflow edge position, due to the presence of the discontinuity. The determination of the ISL is therefore rather based on a larger sample of intensity spectra, which typically includes images without discontinuities. The occurrence of discontinuities in the intensity spectra further justified the implementation of a feature extraction technique that recognized the underflow edges more effectively, namely, motion detection. 


\subsubsection{Motion detection within interval search limits}

The type of motion detection incorporated in the image analysis algorithm is the simplest and quickest form of motion detection (Shafie et al., 2009). This type of detection is based on determining the difference in two consecutive frames, $F_{k}-F_{k-1}$. To determine the difference, the intensity spectrum of a frame was subtracted from that of the next frame. Any resultant intensity differences indicate points of motion. This calculation was limited to the regions within the ISL.

As previously mentioned, the effect of random noise and the need to reduce it, becomes apparent during motion detection. The first two figures in figure 5-10 illustrate the effect of random noise reduction, through contrast and brightness adjustment, on motion detection. In the figure, points of motion are indicated as white pixels.

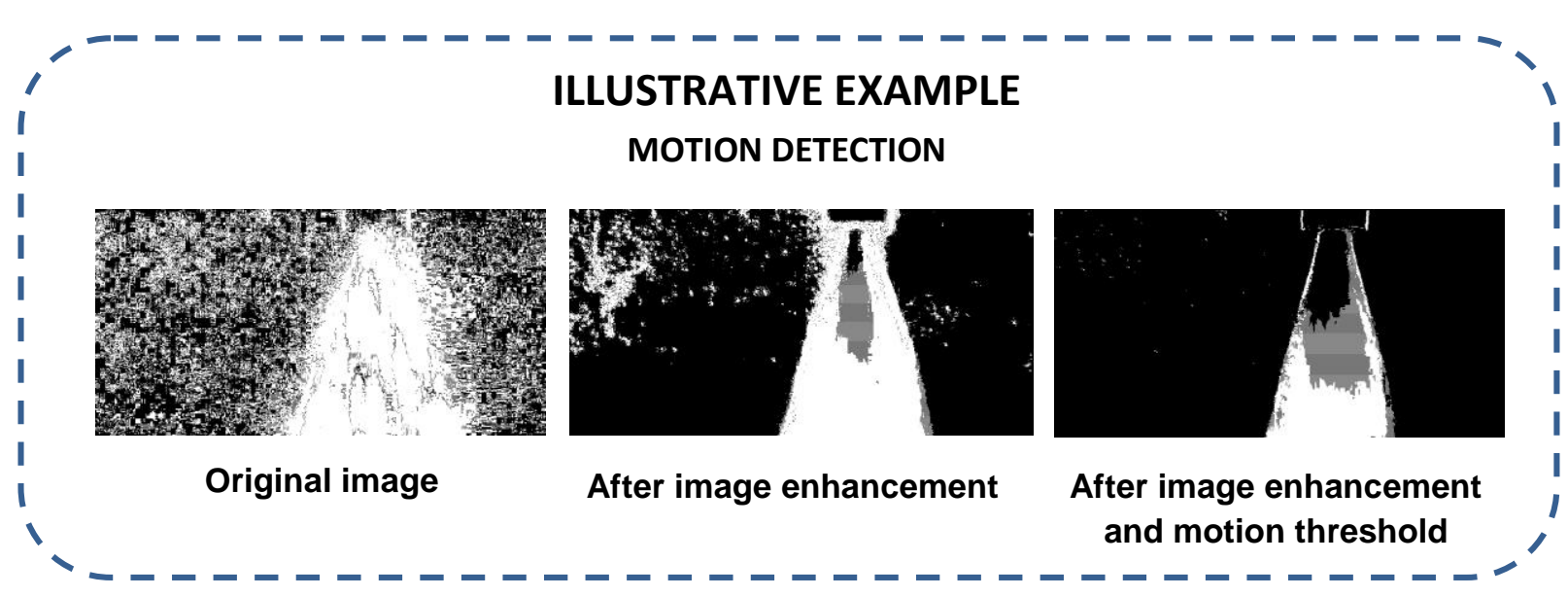

Figure 5-10: Effect of image enhancement and motion threshold on motion detection

Even after applying contrast and brightness adjustments, noise may still be detected in the background of images. Generally, this is because noise cannot be removed completely through image enhancement since it would also lead to distortion or decreased visibility of the underflow stream. Slight system vibration might also cause points of motion in the image background. The motion caused by remaining noise and system vibration is usually less prominent than the underflow motion. To further reduce noise and improve underflow interpretability, a motion threshold was implemented as another user input. The effect of the motion threshold is illustrated in the last picture of figure 5-10.

\subsubsection{Extraction of underflow width}

The first and last points of motion along the horizontal line and within the ISL were identified. Figure 5-11 shows how the ISL serves to eliminate residual noise. The amount of pixels between these two points was calculated as the underflow width for each frame. What is henceforth referred to as underflow width is therefore not an actual measure of length, but rather an indication of the amount of pixels between the underflow edges. 


\section{ILLUSTRATIVE EXAMPLE EXTRACTION OF UNDERFLOW WIDTH}

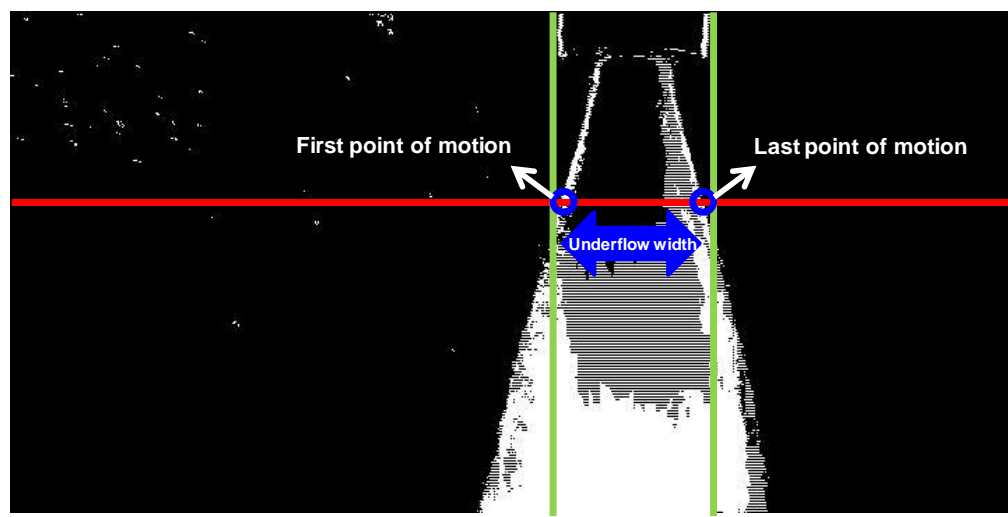

Figure 5-11: Determination of underflow width along specified line and within specified region

\subsection{Diagnostic monitoring technique}

To determine whether operating states could be identified, supporting diagnostic monitoring methods were implemented. The application of these techniques is detailed in the next section. Where necessary, a brief introduction is also given to certain novel concepts.

\subsubsection{Smoothing of time series}

After compiling the underflow width data for an entire case study, the trend was highlighted and overall variance reduced by applying smoothing. Smoothing is a standard technique employed in time series trend analysis (Statsoft Inc., 2010). In this study, the smoothing effect was realized by calculating the simple moving average of every 30 frames (equivalent to 1 second of data). Figure 5-12 illustrates the smoothing effect on a section of the time series from the Merensky 1 case study.

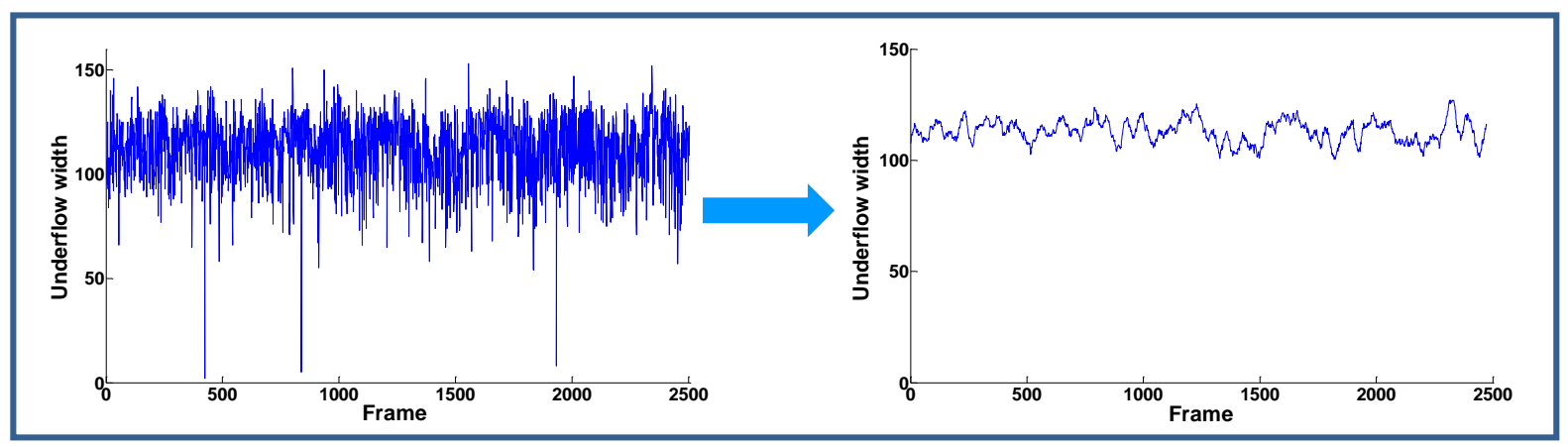

Figure 5-12: Reduction in underflow width variance through moving average 


\subsubsection{Time series embedding}

Ruelle (1980) and Takens (1981) originally suggested time series embedding as a method to capture system dynamics. The reasoning behind this method, and how it has been applied in this study, is described.

\section{(a) Fundamental concepts}

According to Elsner and Tsonis (1996), one variable in a dynamical system can contain information regarding the interaction of other variables, and can therefore be used to represent the system dynamics. Mathematically, this can be illustrated by regarding a dynamical system as a set of $p$-th order differential equations:

$\frac{d \beta_{1}}{d t}=f_{1}\left(\beta_{1}, \beta_{2}, \ldots, \beta_{p}\right)$

Equation 5-2

$\frac{d \beta_{2}}{d t}=f_{2}\left(\beta_{1}, \beta_{2}, \ldots, \beta_{p}\right)$

$\cdots$

$\frac{d \beta_{p}}{d t}=f_{p}\left(\beta_{1}, \beta_{2}, \ldots, \beta_{p}\right)$

Essentially, the dynamical system can also be represented as one sequentially differentiated equation of the $p$-th order:

$\frac{d^{p} \beta_{1}}{d t}=f\left(\beta_{1}, \frac{d \beta_{1}}{d t}, \frac{d^{2} \beta_{1}}{d t^{2}}, \ldots, \frac{d^{p-1} \beta_{1}}{d t^{p-1}}\right)$

Equation 5-3

Ruelle (1980) suggested that system dynamics cannot only be represented by a sequentially differentiated variable, but also by a time series and sequential shifts of the embedding lag, $\tau$. This notion effectively suggests that the shifting of the embedding lag is equivalent to differentiation.

The vector obtained through embedding is of the form $u(t)=\{w(t), w(t+\tau), \ldots, w(t+(d-1) \tau)\}$, with $w(t)$ representing the time series value at time $t$ and $d$ representing the embedding dimension. Based on this vector a space can be constructed that captures the system dynamics. Such a reconstructed space is typically referred to as a phase space. The dimensionality of the phase space, $d$, is determined by the number of lags used. 


\section{(b) Estimation of embedding lag through average mutual information (AMI)}

Fraser and Swinney (1986) have proposed the use of AMI for the estimation of the embedding lag. To date, AMI is one of the most common techniques used in this regard (Manabe and Chakraborty, 2007).

Essentially, the mutual information between two random variables gives an indication of the amount of information shared between the variables. The AMI is mathematically defined as follows:

$I(\tau)=\sum_{w(t), w(t+\tau)} P(w(t), w(t+\tau)) \times \log _{2} \frac{P(w(t), w(t+\tau))}{P(w(t)) P(w(t+\tau))}$ Equation 5-4

where

$I(\tau)=$ average mutual information

$P(w(t), w(t+\tau))=$ joint probability density function of $w(t)$ and $w(t+\tau)$

$P(w(t))=$ probability denstiy function of $w(t)$

$P(w(t+\tau))=$ probability denstiy function of $w(t+\tau)$

The probability functions are determined from the histograms of the respective time series. A maximum in $I(\tau)$ implies that $w(t)$ and $w(t+\tau)$ are strongly correlated (or dependent). On the contrary, a minimum in $I(\tau)$ is associated with strong decorrelation and indicates that the two variables are independent. During time series embedding, $w(t)$ and $w(t+\tau)$ become decorrelated as $\tau$ is increased. The optimal embedding lag is defined as the lag for which the first minimum in $I(\tau)$ is detected.

In this study, the estimation of the optimal embedding lag through AMI was implemented through the predefined MATLAB ${ }^{\mathrm{TM}}$ tool, Quick-Ident*. The entire time series for each case study served as input to Quick-Ident for lag estimation.

\section{(c) Estimation of embedding dimension}

With regards to the embedding dimension, it is common practice to estimate an optimal $d$ through the application of certain heuristic methods (Manabe and Chakraborty, 2007). However, based on preliminary inspections of constructed phase spaces, if was found that a two-dimensional phase space sufficiently captured system dynamics (refer to section 6.3.1). Optimization of the embedding dimension was therefore not incorporated in the monitoring technique.

* Created by Dr. J.P. Barnard, Centre of Process Monitoring, Stellenbosch 


\subsubsection{Support vector machines (SVM)}

Support vector machines (SVM) are considered a powerful machine learning tool for fault diagnosis. Although computationally more expensive than other machine learning techniques, SVM perform particularly well with regards to classification problems and are therefore considered to be more robust (Navia-Vázquez and Parrado-Hernández, 2005). SVM were therefore an obvious choice as machine learning tool for this study.

\section{(a) Fundamental concepts}

The primary objective of SVM is to define a hyperplane that separates different classes of data. In general though, the separation of two classes in the unadjusted input space is not possible through a linear hyperplane. The input space is therefore transformed by mapping the data into a higher dimensional feature space where class separation through a linear hyperplane is made possible: $u=\left(u_{1}, \ldots, u_{m}\right) \rightarrow \varphi(u)=\left(\varphi_{1}(u), \ldots, \varphi_{m}(u)\right)$. This transformation is achieved through the implementation of kernel functions (Shin et al., 2005).

The resultant hyperplane is then observed as a non-linear boundary in the original input space. Effectively the hyperplane functions as a decision boundary: any points inside the boundary are predicted to belong to one class while points outside the boundary belong to another class. The term 'decision boundary' will henceforth be used when referring to a hyperplane. An overview of the SVM transformation process is schematically illustrated in figure 5-13.

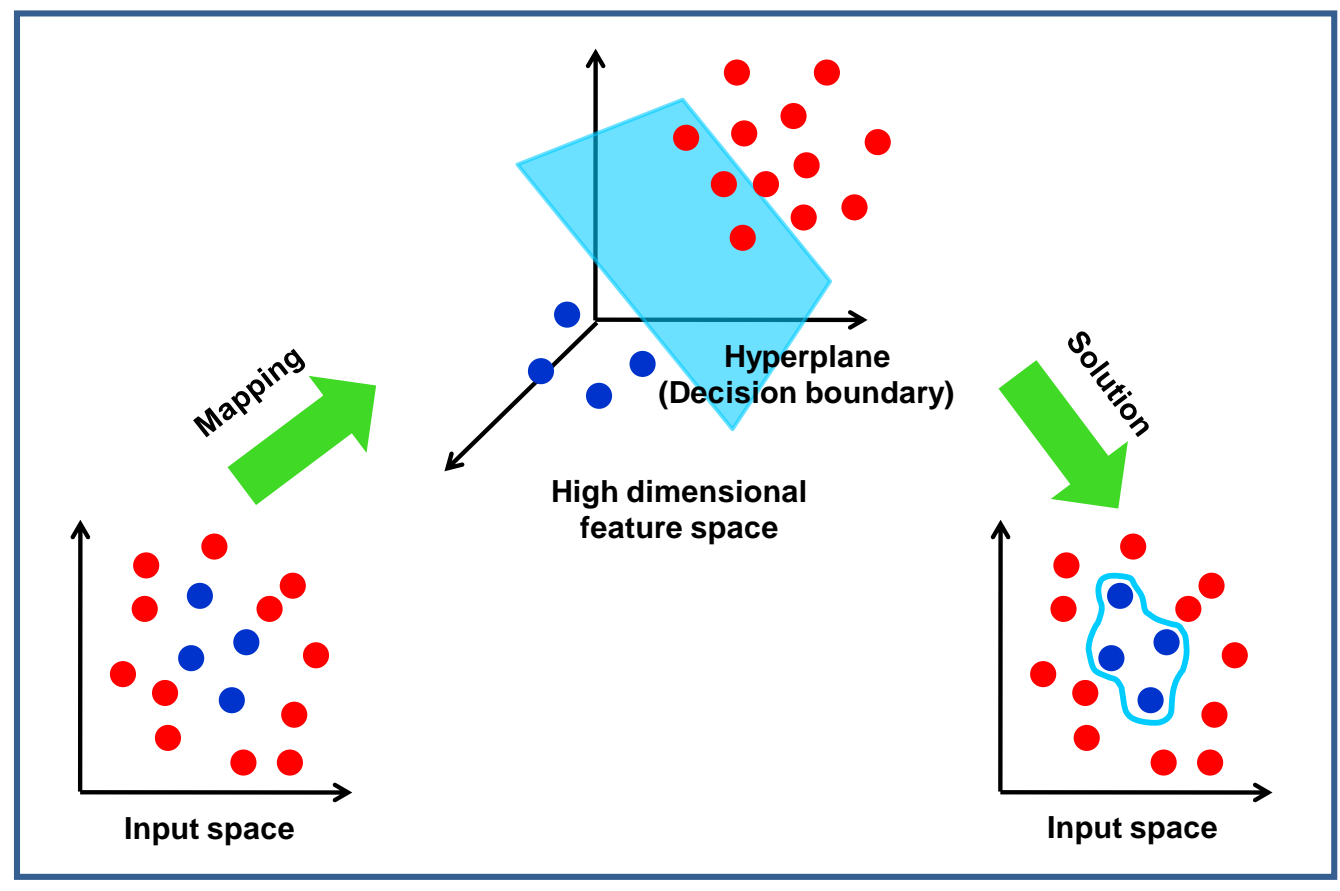

Figure 5-13: Overview of SVM transformation process 
In addition to determining a decision boundary, another purpose of SVM is to optimize the position thereof (Shin et al., 2005). To illustrate this concept, figure 5-14 depicts the implementation of a non-optimal and optimal decision boundary for similar data groups. Effectively, larger boundary margins lead to more optimal separations. The data points that restrict the width of the margin are consequently referred to as the SVM.

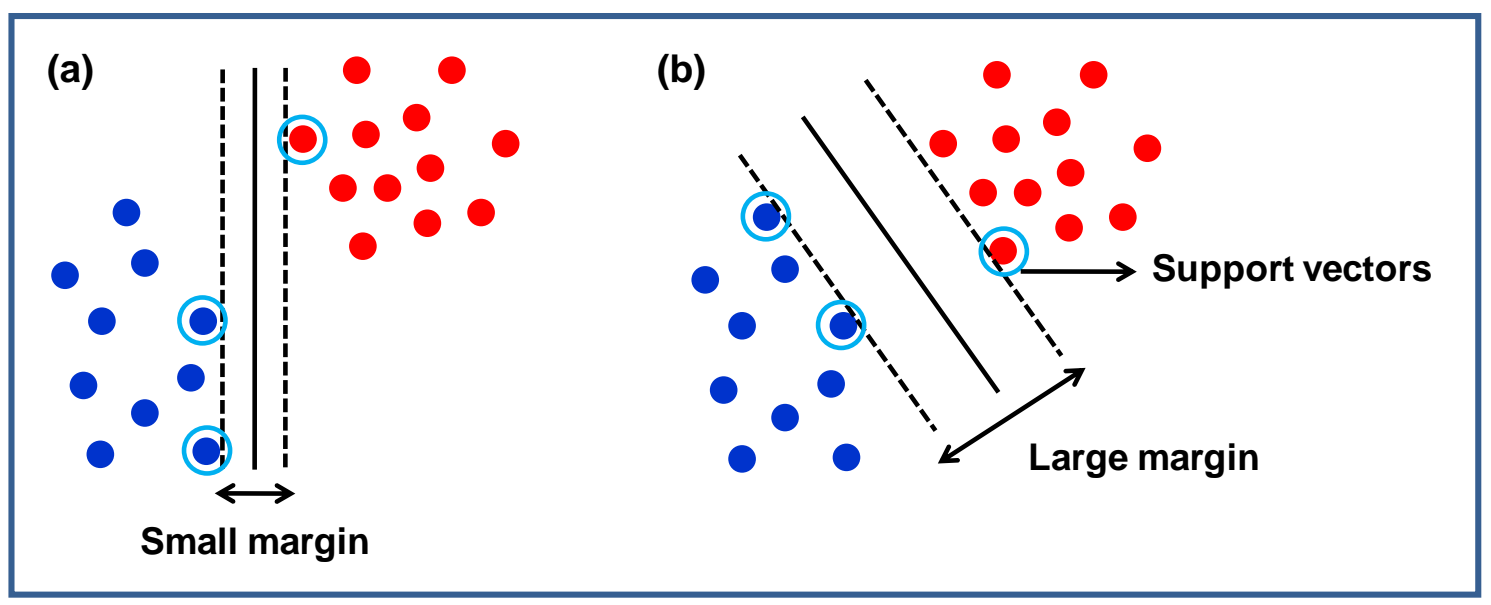

Figure 5-14: Comparison of (a) non-optimal and (b) optimal decision boundary

\section{(b) One-class support vector machines}

In the usual application of SVM, examples of positive as well as negative data are required to train the model. For the case where only positive data is available, Schölkopf (1999) suggested a modified version of SVM, namely, one-class SVM. Since only one class is provided, one-class SVM are considered an unsupervised learning technique. The purpose of one-class SVM is to recognize data belonging to the specified class and to distinguish it from all data points from other classes by classifying them as outliers. This is implemented by specifying the origin of the feature space as the only member of the second class (or negative data), as shown in figure 5-15.

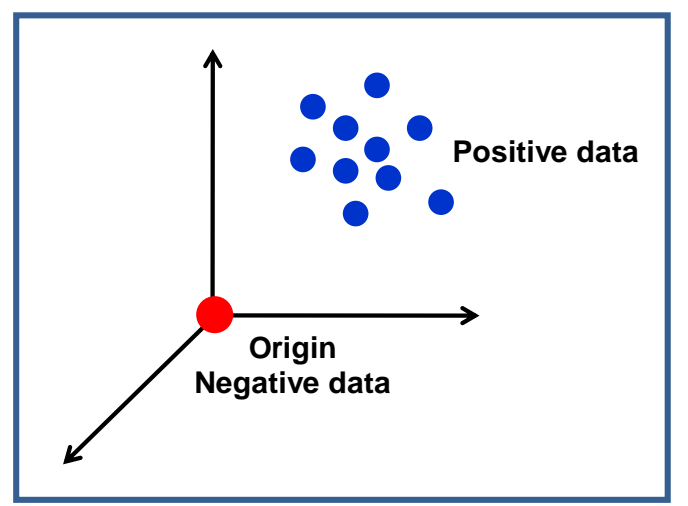

Figure 5-15: One-class SVM classification in feature space 
In the case of one-class SVM, the decision boundary position is determined by equation 5-5 (Shin et al., 2005):

$\min _{\omega, \varepsilon_{i}, \rho} \frac{1}{2}\|z\|^{2}+\sum_{i=1}^{m}\left(\varepsilon_{i}-v \rho\right)$

Equation 5-5

subject to

$\left\langle z \cdot \varphi\left(u_{i}\right)\right\rangle \geq \rho-\varepsilon_{i} ; \quad \varepsilon_{i} \geq 0 ; \quad i=1, \ldots, m$

with

$w=$ margin of the decision boundary,

$\omega=$ weight vector,

$\varepsilon_{i}=$ slack variable,

$\rho=$ threshold level,

$v=$ error limits and

$\varphi\left(u_{i}\right)=$ non-linear mapping function of original input data point $u_{i}$.

The kernel employed during the study is the Gaussian kernel, which is defined by equation 5-6 (Shin et al., 2005):

$K\left(u_{i}, u_{j}\right)=\exp \left(-\gamma\left\|u_{i}-u_{j}\right\|\right)^{2}$

Equation 5-6

subject to

$\gamma>0$

where

$\gamma=$ kernel bandwidth and

$u_{i}$ and $u_{j}$ are orthogonal vectors.

\section{(c) Optimization of kernel bandwidth, $\gamma$}

The kernel bandwidth is an important parameter specification required for the implementation of one-class SVM. To automate the selection of an optimal kernel bandwidth, the following procedure was employed (Chang and Lin, 2001):

First of all, the training data (representing positive data or normal operating conditions) was specified. In terms of hydrocyclone operating states, the training data corresponds to the transition state. Underflow widths that were attained during this operating state were therefore used as training data.

Thereafter, five-fold cross validation was performed on the training data. The cross validation entailed dividing the training data into five subsets. One subset was used to train 
a one-class SVM model and tested against the other four subsets. This process was repeated for the tested kernel bandwidths ranging from $2^{-19}$ to $2^{9}$. To automatically select an optimal value, the following criteria were specified:

- a minimum average accuracy of $90 \%$ over the five cross-validation models;

- less than $10 \%$ of the training data should be used as support vector machines.

The smallest $\gamma$-value that satisfied both criteria, was selected as the optimal. The particular criteria were specified as it generally results in visually pleasing decision boundaries that are neither under- or overfitted.

\section{(d) Training of one-class SVM model}

By incorporating the optimal kernel bandwidth, the entire training data set was used to train the one-class SVM model. This enabled subsequent classification of unseen data points. The one-class SVM modelling technique described in sections 5.3.3 (b) and (c) was implemented by use of the third-party software, LIBSVM* (Chang and Lin, 2001).

\subsection{Conceptual calibration and on-line monitoring algorithms}

Based on the detailed techniques, conceptual calibration and on-line monitoring algorithms are presented for real world application. An overview is presented for each of these algorithms.

\section{(a) Calibration algorithm}

Figure 5-16 depicts the conceptual calibration algorithm described in the next section:

For calibration, a sample video segment would typically be used to determine the user inputs required for image analysis. After setting the user inputs, calibration would commence based on the calibration set $F_{k}$ where $k=1,2, \ldots N$. As each image (frame) is recorded, it would be imported to the computing system. Thereafter greyscale conversion, image enhancement, horizontal line extraction and intensity spectrum generation would follow.

For every 1800 frames, the intensity spectra would be used to determine ISL. The ISL would then be applied to the subsequent 1800 frames. The ISL update rate of 1800 frames relates to 1 minute of video data (this corresponds to the update rate used during this study). If desired, this rate can be changed. Motion detection would consequently be performed and restricted to the ISL. Finally, the underflow width would be extracted.

* Modified for specific application of this study by Dr. L. Auret, University of Stellenbosch 
When the underflow width for the final image, $F_{N}$, is determined, smoothing would be applied to the entire data set. Through application of average mutual information, an optimal embedding lag would then be estimated and consequently used to generate a phase space. If the calibration data does not only contain positive data at this stage, but also fault conditions, it would be necessary to specify the data on which the one-class SVM model should be trained. This specification is the last user input required during calibration. Based on the training data, an optimal kernel bandwidth would automatically be detected. Subsequent training of the one-class SVM model would then take place.

The method used during this study, is very similar to that described in the calibration algorithm. The only difference is that the image data was imported as several separate video segments. After image analysis was applied to each segment, the underflow width data of all the segments were compiled. Subsequent diagnostic monitoring was performed exactly as describe in the calibration algorithm. It should be noted that the user inputs defined in the algorithm remained constant for an entire case study. These inputs did differ from case study to case study, depending on the camera position, amount of noise and overall image quality. A summary of the user inputs for all the case studies is provided in appendix C-2.

\section{(b) On-line monitoring algorithm}

The conceptual on-line monitoring algorithm is provided in figure 5-17 and described in the next section:

Since user inputs are determined and specified during calibration, no inputs would be required for the on-line monitoring process. Theoretically speaking, on-line monitoring would be performed indefinitely, and the data set is therefore denoted as $F_{k}$ with $k=$ $1,2, \ldots \infty$.

The on-line monitoring algorithm closely resembles the calibration algorithm up to the extraction of the underflow width. At this point the individual underflow width would be subjected to diagnostic monitoring (unlike calibration where diagnostic monitoring was applied to a whole data set). Data smoothing and phase space generation would consequently be applied to the individual data point. The one-class SVM model, trained during calibration, would then classify this point as either a normal operating point or a fault condition. If a fault condition is detected, an alarm would be signalled. This entire process would be repeated as new images are acquired.

It should be noted that on-line monitoring would be subjected to a brief initialization period of 1 minute to ensure that enough images have been processed to detect the first ISL. Typically, alarms would be deactivated for this period, as faulty readings might be obtained. 


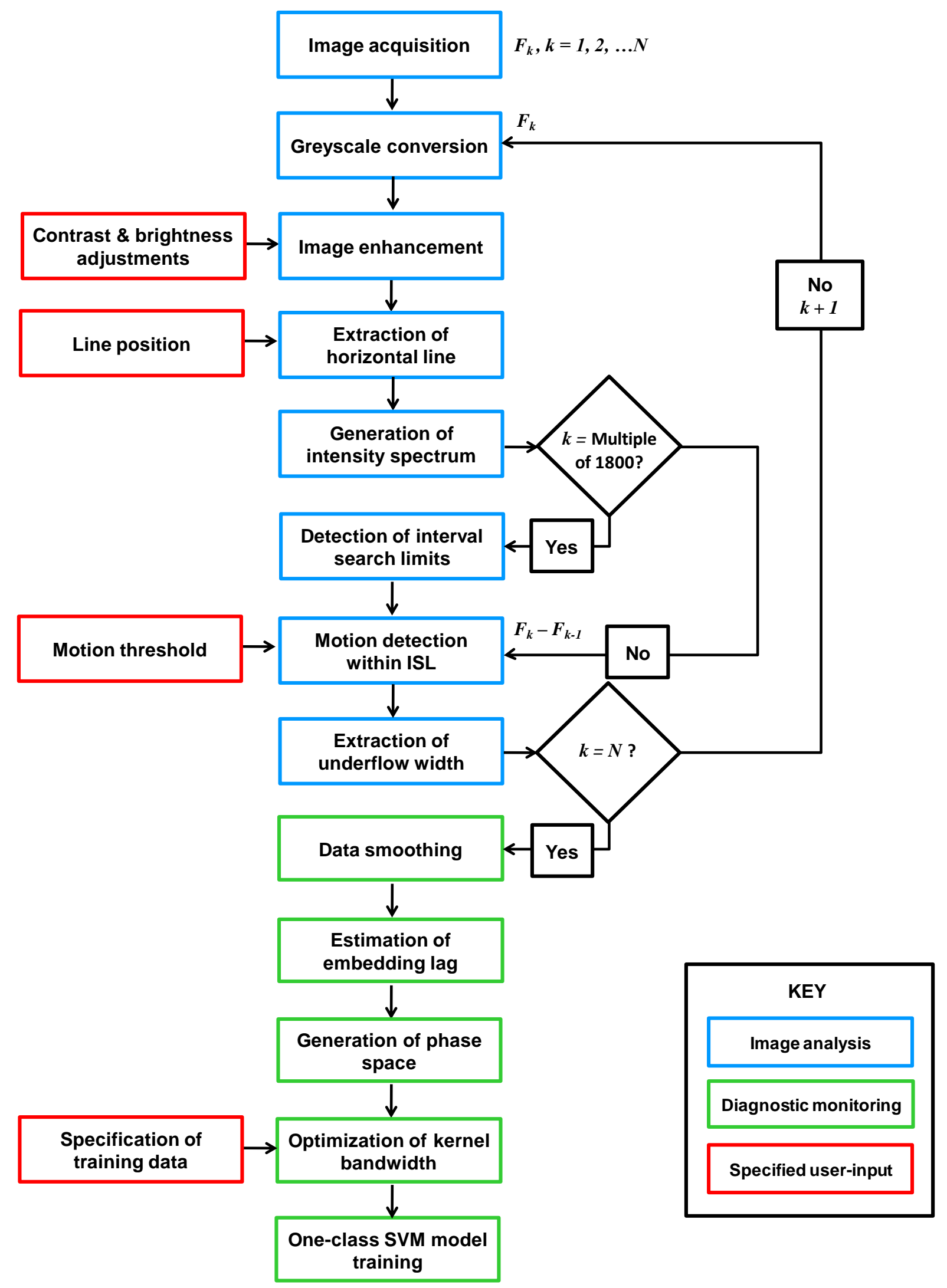

Figure 5-16: Conceptual calibration algorithm 


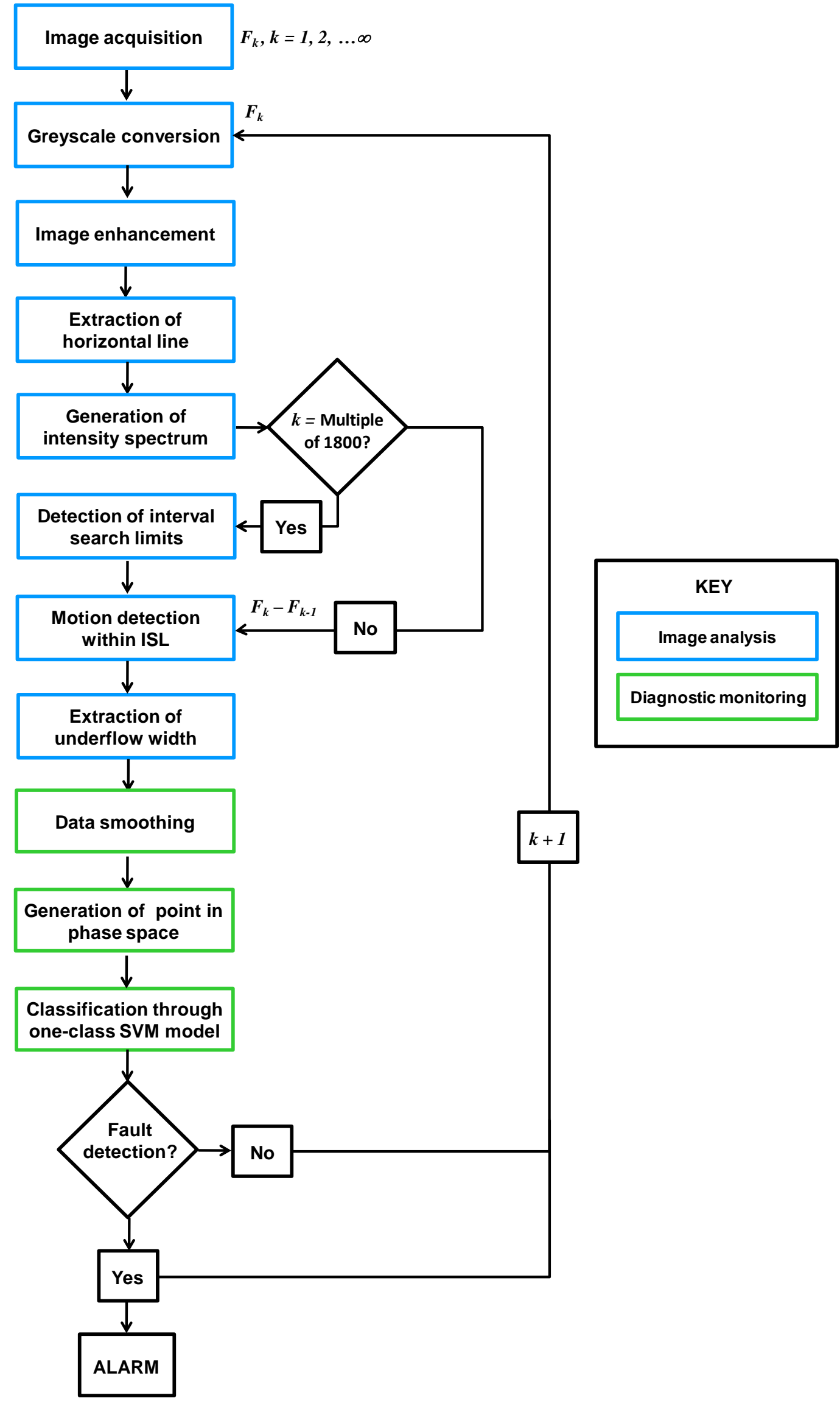

Figure 5-17: Conceptual on-line monitoring algorithm 


\section{CHAPTER SUMIMARY}

As the first stage of image analysis, videos were acquired and separated into individual frames. Each frame was converted to greyscale to reduce processing time and space. Image enhancement, through contrast and brightness adjustment, was then performed to decrease background- and random noise and to increase the interpretability of the underflow stream to the computing system. To reduce processing requirements further, a horizontal line passing through the underflow was extracted and used for subsequent analysis. Based on a compilation of intensity spectra, interval search limits (ISL) were determined. These limits were used to remove foreground- and remaining background noise. Subsequent motion detection was consequently restricted to the ISL. The amount of pixels between the first and last point of motion within the ISL was then calculated as the underflow width. To investigate the extent to which different operating states can be identified, diagnostic monitoring techniques were also applied. This entailed smoothing, followed by time series embedding and finally classification through one-class SVM. In conclusion, overviews are provided for the conceptual calibration and on-line monitoring algorithms. 


\section{CHAPTER 6: RESULTS AND DISCUSSION}

In this penultimate chapter, the final results are presented and interpreted. The first section serves as a preliminary evaluation of the implemented image analysis technique. Thereafter, the image analysis results are investigated by introducing the general trends and comparing results to visual observations. Diagnostic monitoring results are also presented to evaluate the extent to which the operating state can be indentified and classified through the use of one-class SVM. Lastly, a few final comments on the monitoring system are made.

\subsection{Preliminary evaluation of image analysis technique}

During image analysis, various factors were identified to have an effect on image quality. These factors effectively influenced the interpretability of the underflow to the computing system and the accuracy with which the underflow width was determined:

- contrast difference;

- background noise;

- foreground noise;

- random noise;

- system vibration.

In the following section, the importance of each of these factors is assessed. The extent to which the developed image analysis technique addresses these factors is also discussed. These findings, which are used to assess the merit of the implemented methods, consequently serve as a preliminary evaluation of the image analysis technique. To give a qualitative indication of the prominence of each factor in the various case studies, a relative comparison is presented in table 6-1.

Table 6-1: Relative comparison of factor prominence in each case study

\begin{tabular}{|c|c|c|c|c|c|}
\hline Case study & $\begin{array}{c}\text { Contrast } \\
\text { difference }\end{array}$ & $\begin{array}{c}\text { Background } \\
\text { noise }\end{array}$ & $\begin{array}{c}\text { Foreground } \\
\text { noise }\end{array}$ & $\begin{array}{c}\text { Random } \\
\text { noise }\end{array}$ & $\begin{array}{c}\text { System } \\
\text { vibration }\end{array}$ \\
\hline Gold & Medium & High & Low & High & Low \\
\hline Ilmenite & Low & Medium & Low & High & Low \\
\hline Platreef & Medium & Low & Low & Low & Medium \\
\hline Merensky 1 & Medium & High & Medium & Low & High \\
\hline Merensky 2 & High & Medium & High & Medium & High \\
\hline Merensky 3 & High & High & Medium & Low & High \\
\hline
\end{tabular}




\subsubsection{Contrast difference}

In order for the computing system to recognize the underflow stream, a contrast difference between the intensities of underflow and the surrounding environment is required. Image enhancement (through contrast and brightness adjustments) was consequently introduced to improve the existing contrast difference of underflow images.

In general, image enhancement resulted in drastic improvement of the contrast difference. As example, intensity plots from the Gold case study are provided in figure 6-1 (a) and (b). By comparing the intensity plots before and after image enhancement, it is found that the difference between the average underflow intensity and average environmental intensity increased with a value of 73 . Relative to the other case studies, the Gold case study is classified to have a medium contrast difference between the underflow and surrounding environment (refer to table 6-1).

(a)

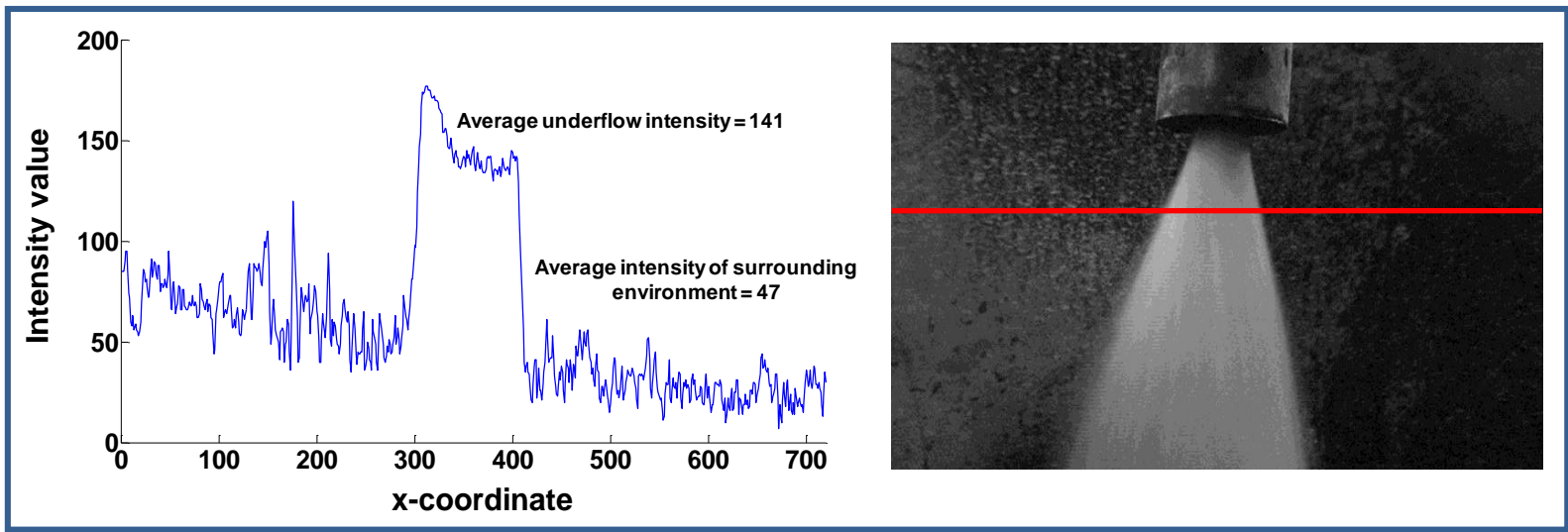

(b)

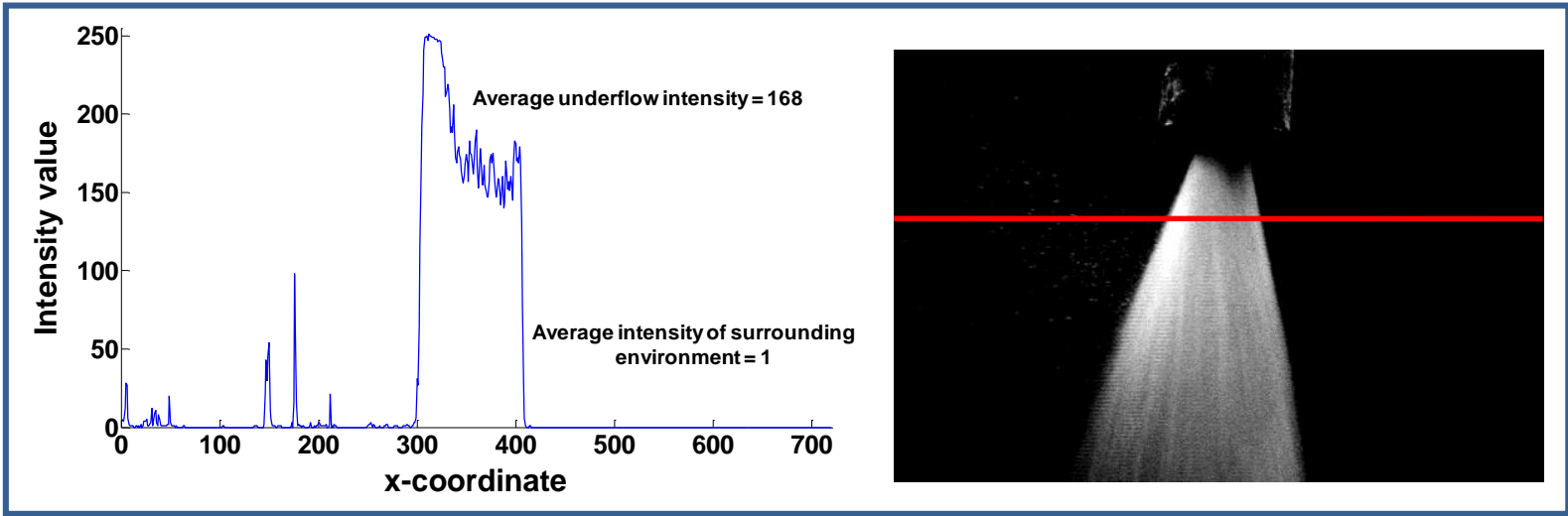

Figure 6-1: Gold intensity plots for specified horizontal line and accompanying underflow images (a) before and (b) after image enhancement

However, image enhancement did not prove effective for the Ilmenite case study with the lowest amount of contrast difference (as indicated in table 6-1). Through investigation of an intensity plot from this case study (provided in figure 6-2), it becomes clear that the underflow cannot be distinguished based on the intensity values. This is due to the fact that the colour of the underflow and the background is too similar. Shadows furthermore 
contribute to the dark colour of the background. Since image enhancement requires that the underflow should at least be recognizable from the intensity plot, subsequent image enhancement serves no purpose. Recognition of the underflow through motion detection alone was also attempted, but failed since intensity differences were also too low to distinguish underflow motion. Based on these findings, the Ilmenite case study was rejected and not subjected to further analysis.

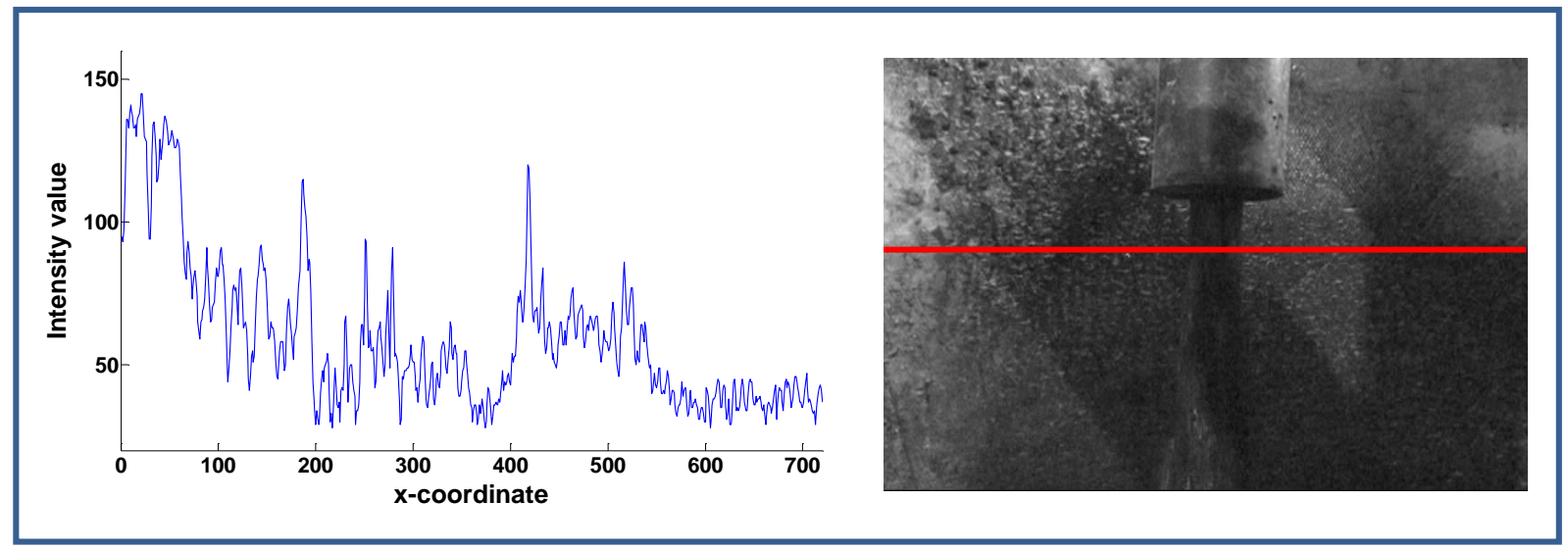

Figure 6-2: Ilmenite intensity plot for specified horizontal line and accompanying underflow image

The use of lighting, in Merensky 2 and 3 case studies, resulted in a higher contrast difference than for the Merensky 1 case study (without any lighting), as suggested in table 6-1. The increase in contrast with the use of lighting might be attributed to the reflective slurry set against the non-reflective background of the mixing tank. Although a sufficient contrast difference was achieved for most case studies without lighting, the latter results suggest that it is beneficial to always incorporate lighting. Another alternative is to position a light to provide backlighting to the underflow. If successfully employed, the silhouette image of the underflow will essentially be recorded. Sufficient contrast difference will hereby already be achieved during recording, independent of the slurry colour. The use of backlighting, however, remains untested in the current study.

\subsubsection{Background noise}

Background noise is introduced by slurry droplets that adhere to the side of the mixing tank. As more droplets collect against the side of the mixing tank, the colour of the background scenery progressively starts to resemble that of the underflow. The consequence is a decrease in contrast difference between the underflow and surrounding environment. Except for this concern, background noise might also accidentally be recognized as part of the underflow. 
Image enhancement was consequently also implemented for the treatment of background noise. Through re-examination of figure 6-1 (a) and (b), it is seen that the background noise is significantly reduced through the contrast and brightness adjustments. The intensity plot of the enhanced image (figure 6-1 (b)) does, however, show a small amount of remaining background noise. Further reduction of this noise was enforced by applying the ISL and motion detection threshold.

Through the implementation of the mentioned techniques, satisfactory background noise reduction was achieved. This is true for all case studies, including the Gold and Merensky 2 and 3 case studies with the largest amount of background noise (as indicated in table 6-1). For the few instances where background noise was not removed completely through the mentioned techniques, the remaining noise was usually represented by a very small region inside the ISL. Such noise inflated the underflow width only slightly (typically an increase of one or two pixel lengths).

Even though the image analysis technique proves effective for the treatment of background noise, it is envisaged that the noise can be removed completely by using backlighting, as previously discussed.

\subsubsection{Foreground noise}

The primary concern with foreground noise is that it could mistakenly be identified as part of the underflow and thereby erroneously inflate the underflow width. Unlike background and random noise, this type of noise is generally not reduced through image enhancement. As a matter of fact, image enhancement actually aggravates foreground noise by enhancing it in a similar way as the underflow. This is because foreground noise consists of high intensity regions (of similar intensity to that of the underflow) and occupies relatively large regions of the image.

To mitigate the effects of excessive foreground noise, underflow recognition was limited to a narrower region through detection of the ISL. Essentially, this would eliminate foreground noise outside the ISL. However, for cases where foreground noise overlaps with the underflow edge limits, the noise is still recognized as part of the underflow and included within the detected limits. Updating the ISL more regularly might prove useful, only if foreground noise is also removed more often.

An example of how a droplet overlapping with the underflow edge can mistakenly increase the underflow width, is illustrated for the Merensky 1 case study in figure 6-3. In this case the underflow width is increased with a value of 11 pixel lengths. 

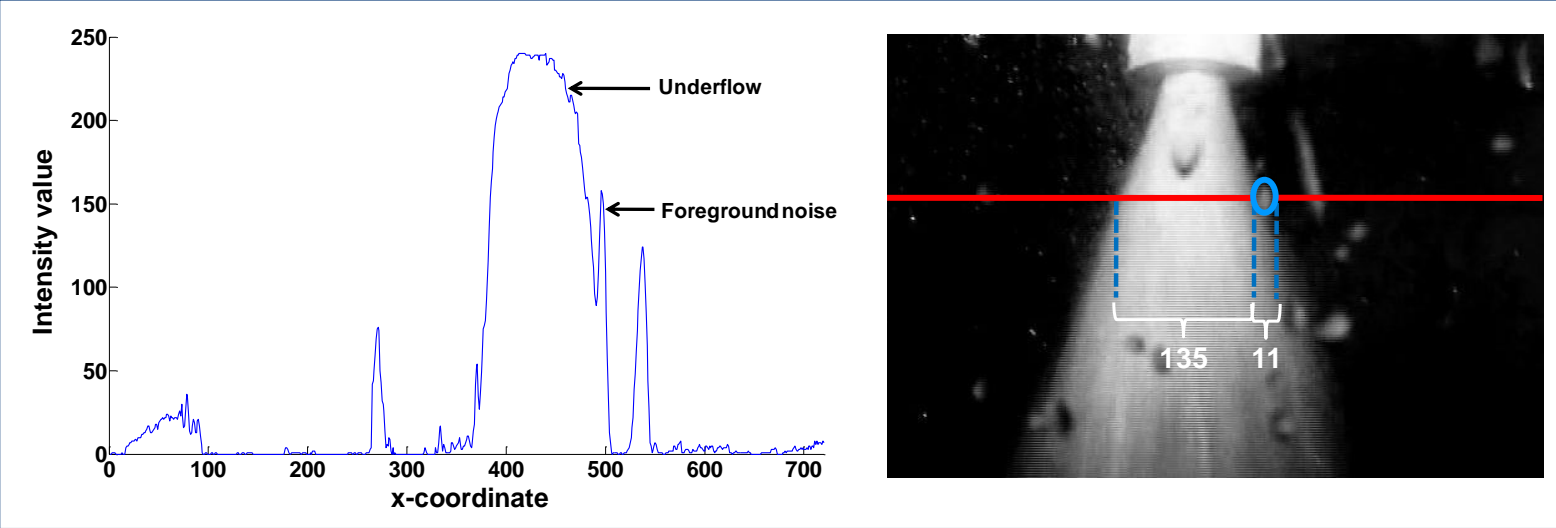

Figure 6-3: Merensky 1 intensity plot for specified horizontal line and accompanying underflow image

The Merensky 1 case study is also the case study with the greatest amount of foreground noise. This is mainly attributed to the fact that the video camera was positioned closer to the underflow compared to the other case studies (due to spatial limitations at the time). As further discussed in sections 6.2.2 (c) and 6.3.3 (c), the presence of foreground noise influenced the underflow width to such an extent that it interfered with the identification of the operating state.

Foreground noise was also detected as points of motion during motion detection, since system vibration of prominent foreground features was difficult to distinguish from underflow motion. In terms of these findings, the techniques used for the reduction of foreground noise are considered insufficient. For the other case studies, though, foreground noise is far less prominent and found not to influence the underflow width significantly.

Since foreground noise manifests as such a noticeable feature, the reduction thereof through image analysis is complicated. Even in the case where foreground noise could be identified successfully, underflow width determination may still be compromised if droplets overlap with the underflow edge, because such droplets actually obscure the true underflow position. A practical solution to foreground noise therefore seems far more feasible.

The simplest practical solution would be to position the video camera outside the splash zone and to maintain image quality by relying on the optical zoom capabilities of modernday cameras. If spatial limitations prohibit this, physical means should be employed for removal of droplets from the sightglass. During experimental work, foreground noise was occasionally removed by cleaning the slightglass with every new ore addition. Although foreground noise was removed, the frequency of cleaning was insufficient. To achieve the same effect at higher frequency and without any human input, an air knife might be used. Alternatively, the sightglass might be treated with hydrophobic wax. 


\subsubsection{Random noise}

Random noise proved problematic during motion detection, as it was erroneously classified as points of motion and hence obscured underflow motion.

The developed image analysis technique addressed random noise primarily through image enhancement and the motion detection threshold. These techniques effectively minimized the random noise in all case studies, including the Gold case study with the most noticeable amounts of random noise (refer to table 6-1). The successful treatment of random noise might be attributed to the fact that it presents as very small regions of slight intensity fluctuations. A large amount of the noise is therefore transformed into the desired intensity range through image enhancement. In terms of motion detection, random noise results in small regions of insignificant motion which is easily distinguished from underflow motion through the motion threshold.

Although the presence of random noise does not pose a major problem for the performed case studies, it is still preferred to reduce the amount originally introduced into video footage. One way to do this is by applying ample lighting during the recording stage.

\subsubsection{System vibration}

System vibration causes features, other than the underflow, to be identified as points of motion during motion detection. It further leads to slight variation in the position of the underflow stream, which is undesired since the underflow width is determined along a certain horizontal position.

During motion detection, successful distinction was made between motion caused by system vibration and underflow motion, by applying the motion threshold (except for prominent foreground features). This clear distinction is realized based on the fact that underflow motion is much more prominent than motion caused by system vibration. The slight variations in underflow position due to system vibration also proved to be minor. This effect was further reduced through the application of smoothing.

Disregarding the one case study with extreme foreground noise (Merensky 1 ), the extent to which the implemented image processing steps addressed system vibration is considered sufficient. This is because the influence on the accuracy of the underflow width was negligible.

It should be mentioned, however, that even though system vibration was easily addressed in the performed case studies, it is evident that there is a limit to the amount of vibration allowed. Vibration might be minimized significantly during recording by ensuring that the 
video camera is fixed to a stationary system, separate from the influence of equipment vibration.

\subsubsection{Comments on feature extraction technique}

Originally, the incorporation for motion detection was motivated based on the simplicity of the technique. However, the various factors that influence underflow interpretability during motion detection, does beg the question whether a more complex feature extraction technique, such as edge detection, would be less susceptible to their effect.

Ultimately, edge detection also relies on sufficient contrast (or intensity) differences for the recognition of edges. It is therefore a given that background and random noise will also contribute to the decreased interpretability of the underflow stream during edge detection. Foreground noise will also continue to obscure the true underflow edge position. System vibration will probably not influence the recognition of the underflow width during edge detection though, but will persist to introduce slight variation in the specified line position.

In light of the above, it seems as though the need for noise reduction methods would not necessarily diminish if another feature extraction method was specified. There does however, exist scope to optimize feature extraction by investigating different noise reduction methods. For instance, other standard techniques such as filtering and thresholding might be researched.

\subsection{Underflow width results obtained through image analysis}

The results obtained through image analysis are presented in the next section. Firstly, the general underflow behaviour is related to changes in feed stream concentration. Secondly, the identification of operating states is investigated by comparing image analysis results with visual observations.

\subsubsection{Underflow width versus feed stream concentration}

To explore the relationship between the underflow width results and feed stream concentration, the results from the Gold and Merensky 2 case studies are presented in figure 6-4 (a) and (b) as examples. A complete set of these results are provided in appendix D-1. The method used to manually monitor the feed stream concentration is furthermore described in appendix B-5.

Figure 6-4 (a) and (b) shows a general decrease in underflow width with an increase in feed stream concentration. This trend is consistent with literature descriptions of the behaviour between these two variables (refer to section 2.4). 
(a)

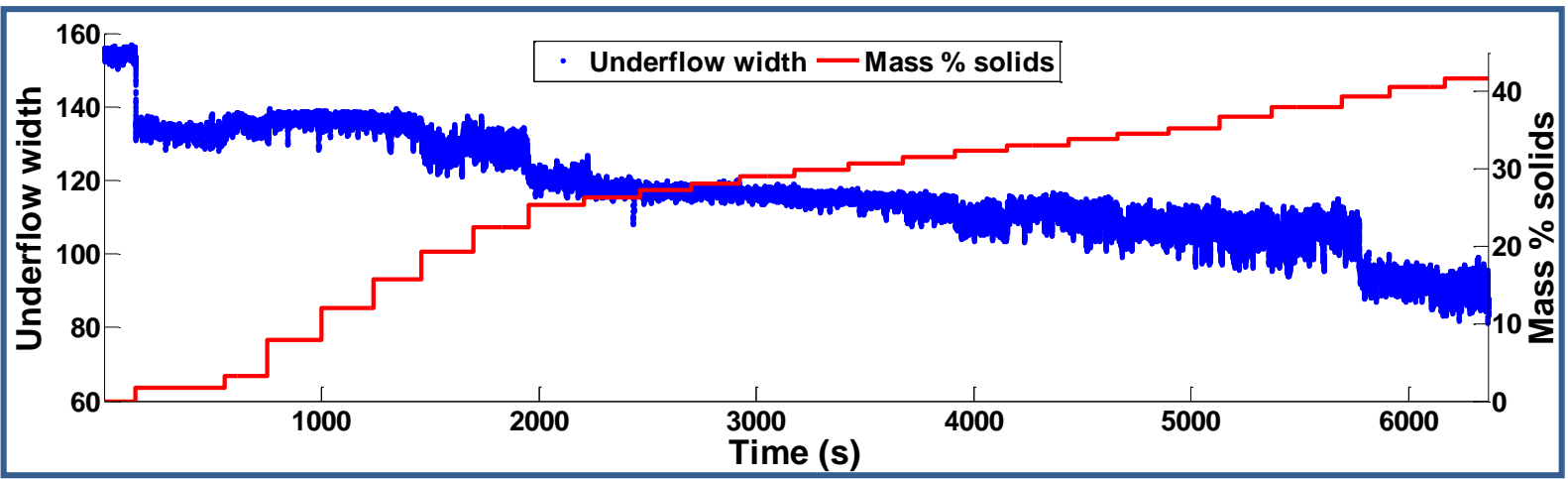

(b)

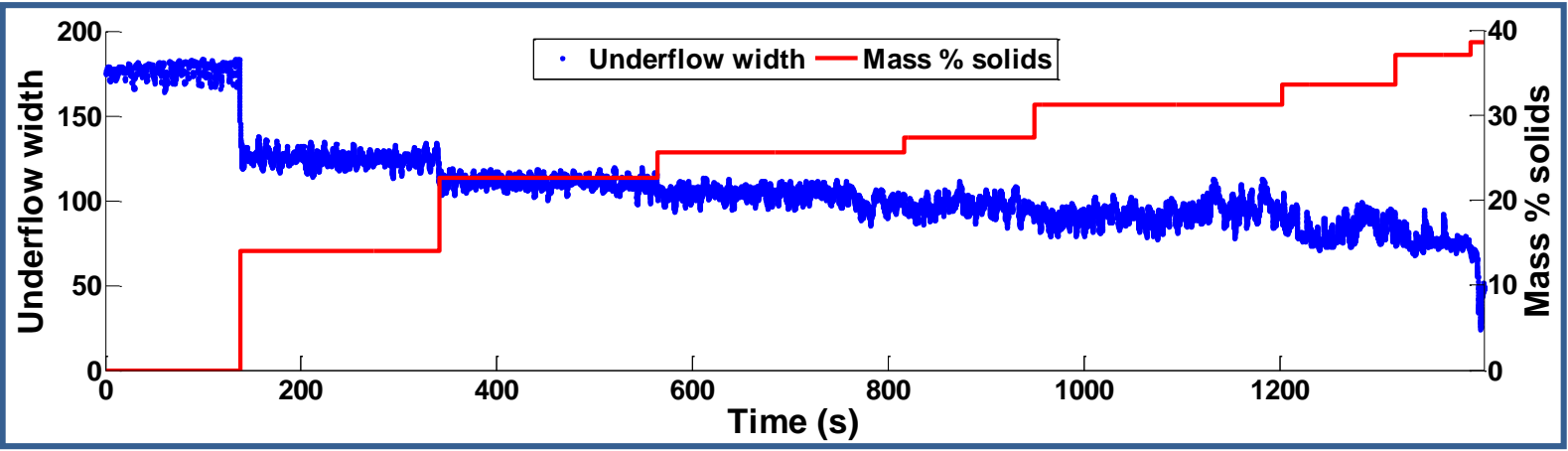

Figure 6-4: Underflow width results against feed stream concentration for

(a) Gold and (b) Merensky 2 case studies

Further investigations of the results reveal a significant drop in underflow width after the first ore addition. This initial drop in underflow width is pronounced, even for a relatively small ore addition, as in the case of the Gold case study (with a feed stream concentration of $1.7 \%$ after the first ore addition). The drastic decrease in underflow width represents the changeover from the initial pure water flow system to a solid-liquid system and the accompanying density increase.

Other, less drastic decreases are witnessed with some of the subsequent ore additions. This is especially true for the Merensky 2 case study for which ore was added in larger increments. A final prominent drop in underflow width is observed at the end of each case study. This final drop suggests the onset of roping discharge.

\subsubsection{Identification of operating states based on visual observations}

Figure 6-5 (a) to (e) illustrates the complete set of underflow width results obtained through image analysis. The exact transition points between the operating states are furthermore indicated for each case study by red lines. These transition points were identified by examining the video footage of each case study*. In the following section, the extent to which the underflow width results serve as indication of the operating states is evaluated.

\footnotetext{
* Since the transition state is characterized by rapid fluctuations between spray and roping discharge, the identification of the respective transition point through normal human observation is dubious. The transition point was therefore identified by observing video footage in slow motion and locating the first fluctuation between spray and roping discharge. In contrast, the onset of roping was easily discernible by the naked eye.
} 


\section{(a) Gold case study}

The transition points identified for the Gold case study, in figure 6-5 (a), are shown to coincide with relatively distinct drops in underflow width. The final drop in underflow width is also confirmed to correspond with roping discharge, as suggested in section 6.2.1.

Slight increases in the underflow width results are noticed during dilute flow separation (approximately between 500 and 1400 seconds), but does not manifest in the video footage. This discrepancy is due to the combined presence of immense background noise and low image contrast at that stage of operation. As the solids concentration increases though, the contrast also increases and the downward trend in underflow width is reinstated, as visually observed.

Increased variation in the underflow width is further noticed during the final stages of the transition state (approximately between 3900 and 5700 seconds). The increased variation corresponds with video footage depicting intensifying fluctuations between spray and roping discharge. Similar increases in variation are also observed in the Merensky 2 and 3 case studies (in figure 6-5 (d) and (e)), but are slightly less pronounced.

\section{(b) Platreef case study}

While the experiment for the Platreef case study was performed, unusual underflow behaviour was witnessed. The underflow discharge was characterized by irregular spurts of slurry. It is expected that this behaviour might be attributed to faulty design of the specific hydrocyclone system. The spurt-like discharge furthermore promoted the build-up of sediment in the hydrocyclone body, causing spigot blocking even at very low solid concentrations.

The aforementioned hydrocyclone behaviour explains the unusual underflow results of figure 6-5 (b). An initial drop in underflow width (at approximately $780 \mathrm{~s}$ to $790 \mathrm{~s}$ ) is shown, followed by a final drop in width at the end (from $850 \mathrm{~s}$ onwards). These results agree with video observations which indicate blocking at the exact times.

Based on both the image analysis results and video observations, no distinction can be made between dilute flow separation and the transition state. This is primarily due to the irregular spurt-like behaviour of the underflow. It is assumed that the majority of larger underflow widths generally represent the transition state and is consequently depicted as such in figure 6-5 (b). 
(a)

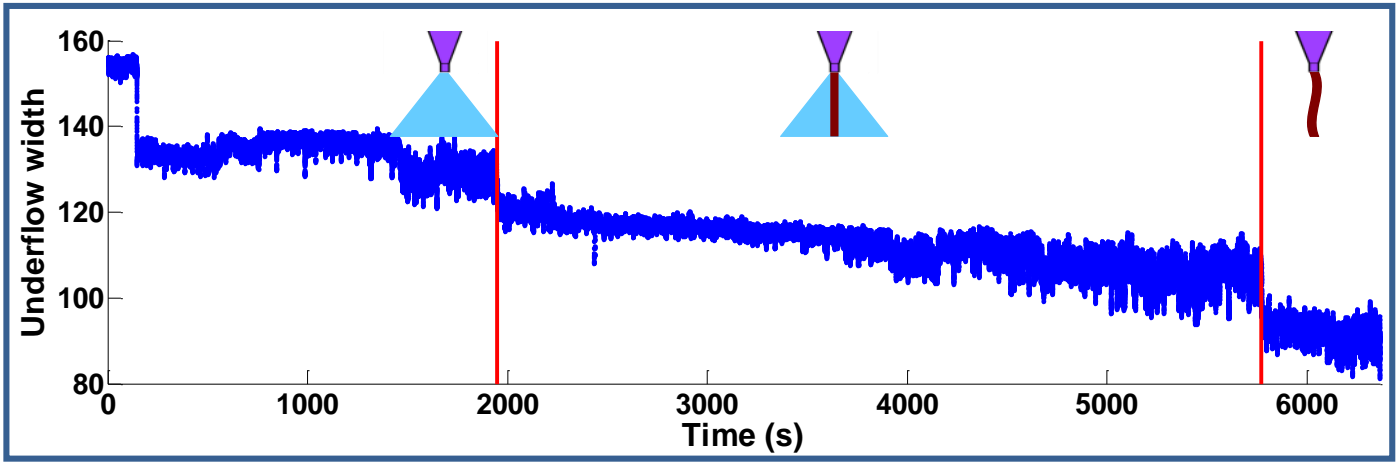

(b)

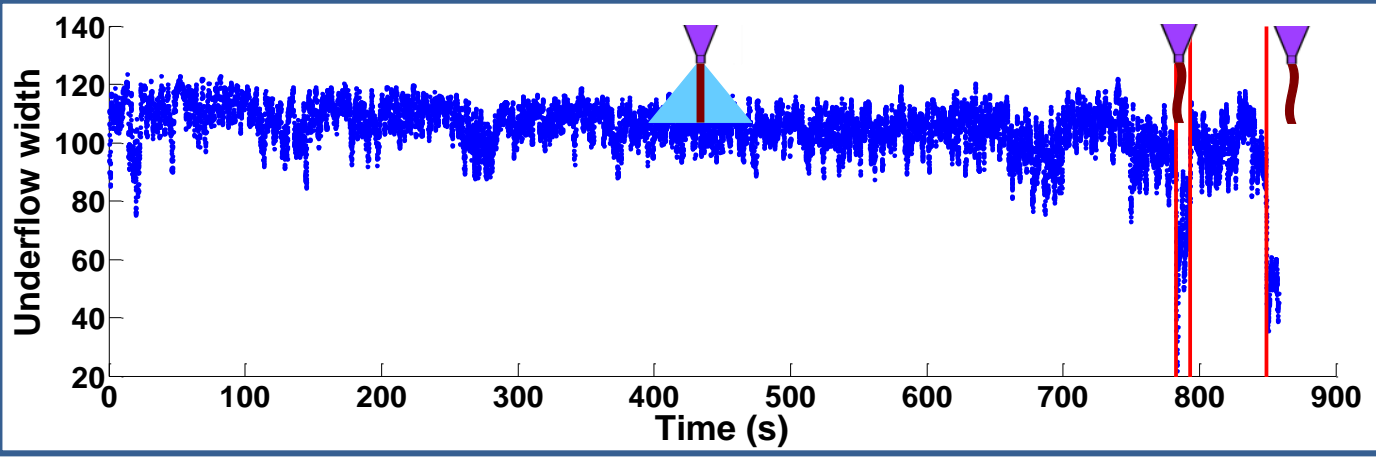

(c)

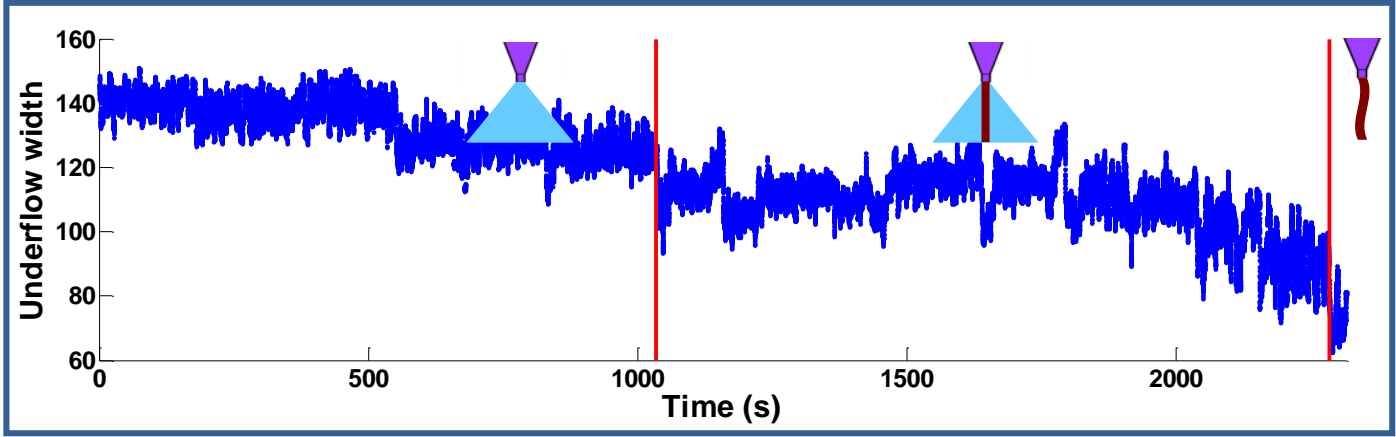

(d)

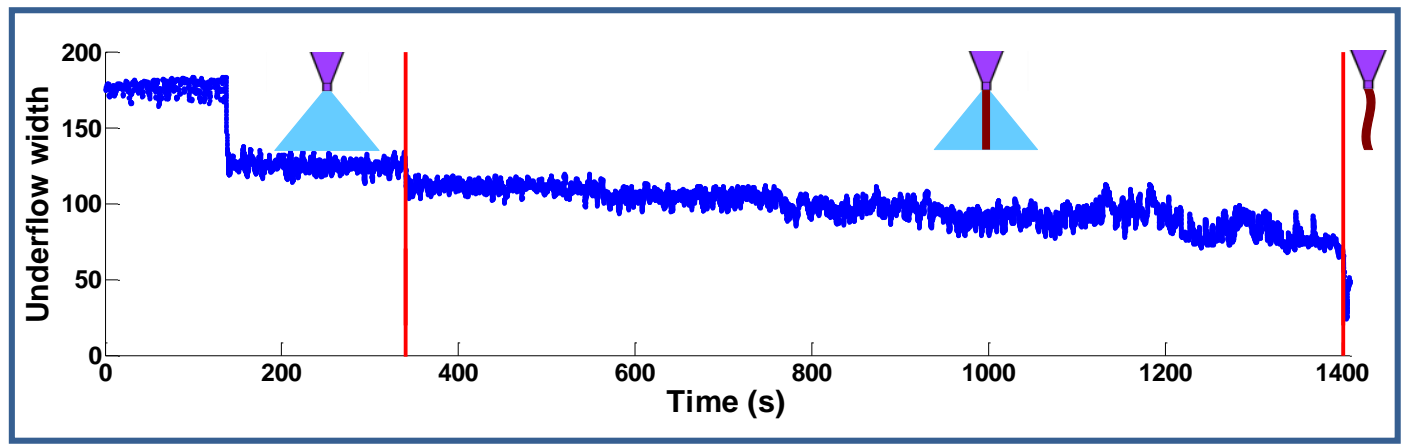

(e)

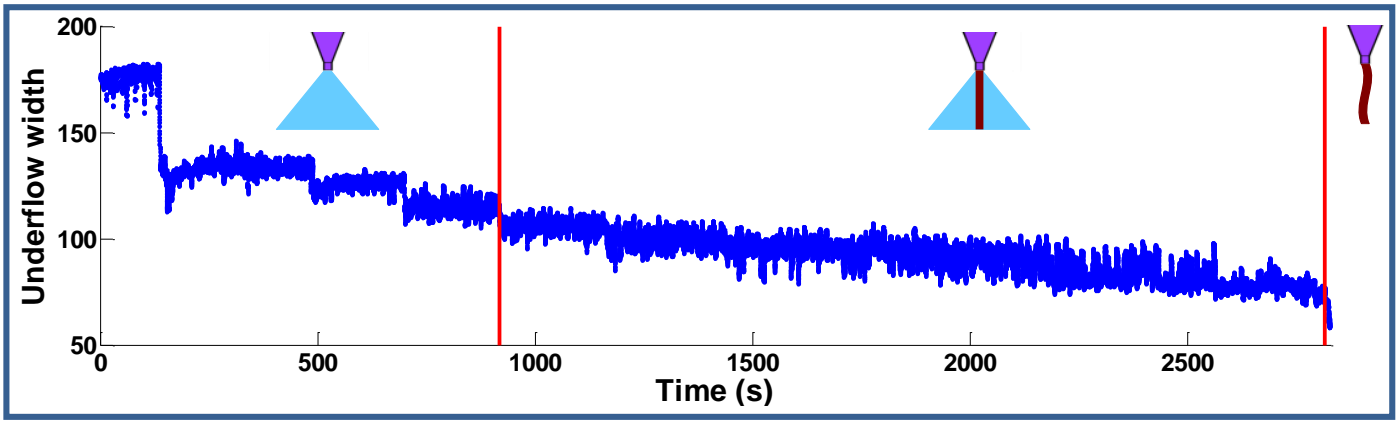

Figure 6-5: Underflow width and identified transition points for (a) Gold, (b) Platreef,

(b) Merensky 1, (d) Merensky 2 and (e) Merensky 3 case studies 


\section{(c) Merensky 1 case study}

An extremely low degree of contrast was witnessed for the initial pure water flow system of the Merensky 1 case study. This may be attributed to the obstruction of natural light at that stage. Consequently, the initial peak in the underflow width results does not feature. The problem was rectified during experimental work by removing the obstruction with the next ore addition.

A general observation for the underflow width results of this case study is that the distinction of operating states is less obvious than in the case of the Gold case study. From figure 6-5 (c), it is furthermore observed that the general downward trend of the underflow is disrupted significantly by irregular variation, especially in the transition state. These irregularities are attributed to extreme foreground noise in the video footage (as previously explained in section 6.1.3).

\section{(d) Merensky 2 case study}

The results of the Merensky 2 case study (shown in figure 6-5 (d)), display trends similar to that of the Gold case study. Transitions between operating states also clearly coincide with decreases in underflow width. The distinction of operating states through underflow width appears even more evident for this case study. This may be contributed to the high degree of contrast achieved for this case study (refer to table 6-1).

\section{(e) Merensky 3 case study}

Since the Merensky 2 and 3 case studies are duplicates, in terms of ore type and video camera position, similarities are expected between the results for the two case studies. A comparison of figure 6-5 (d) and (e) confirms this is in the following manner: As with the Merensky 2 case study, the distinction of operating states from the underflow width is very clear in the Merensky 3 case study. Underflow ranges for each operating state furthermore agree very well with the Merensky 2 case study.

\section{(f) Motivation for application of machine learning through one-class SVM}

For each operating state, a range of underflow widths in which the respective state occurs, can be specified. Essentially, this information can be used to apply univariate monitoring thresholds for the recognition of different operating states. However, the specification of a range unique to each operating state, and the subsequent recognition thereof, is not considered a clear cut monitoring technique. This is because there exists significant overlap between the ranges for each operating state, as illustrated by figure 6-5 (a) to (e). For example, for the Gold case study an underflow width of 100 might belong to ranges 
corresponding to either the transition state or dense flow separation. This causes ambiguity and could result in misclassification of serious fault conditions. A more robust technique is therefore required to distinguish between the different operating states.

Typically, a calibration set will consist of transition state data (also referred to as positive data or normal operating conditions) and not include fault conditions because of the associated consequences. It is therefore desired that a technique be employed that can also successfully identify fault conditions, based on an example set of the transition state only.

These mentioned requirements necessitate a more robust method for the recognition of operating states and thereby motivates the investigation of one-class SVM as a machine learning tool for the specific problem.

\subsection{Diagnostic monitoring of underflow width}

As described in section 5.3.3, certain parameters are automatically selected during the diagnostic monitoring process. These parameters include the embedding lag, $\tau$, and the kernel bandwidth, $\gamma$. As example, the selection of these parameters are illustrated and interpreted for the Gold case study. Thereafter, the final one-class SVM results are presented for all case studies. A comparison is also made to a much simpler classification technique, multivariate Gaussian distribution (MVGD). Finally, an investigation on the sensitivity of the embedding lag is presented.

\subsubsection{Selection of embedding lag, $\tau$, and phase space generation}

A plot of the AMI (average mutual information) for the Gold case study is provided in figure 6-6 (a). The plot indicates how the coherence between the underflow width and the lagged copy decreases as the embedding lag increases. The first minimum is observed at a lag of 29. This sharp decrease in coherence is expected, since the underflow width data is based on the moving average of every 30 points. Due to the fact that decorrelation was intensified at this point through the use of a moving average, 29 was automatically rejected as the true optimal lag. A subsequent minimum in autocorrelation occurs at 132, as illustrated in figure 6-6 (a). This minimum represents a decrease in autocorrelation which is inherent to the original data and therefore identified as the optimal embedding lag.

A phase space, based on a lag of 132, was consequently generated and is illustrated in figure 6-6 (b). Several clusters are revealed in the resultant phase space. Based on the previously defined transition points, it was found that these clusters represent the various operating states. Dilute flow separation occupies the top right-hand corner of the phase space while dense flow separation corresponds to the clusters in the bottom left-hand corner. The transition state is situated between the clusters of dilute and dense flow separation. It 
should be noted that dilute flow separation actually manifests as two separate clusters: pure water flow with distinctly higher underflow widths and the remainder of dilute flow separation characterized by lower underflow widths.

(a)

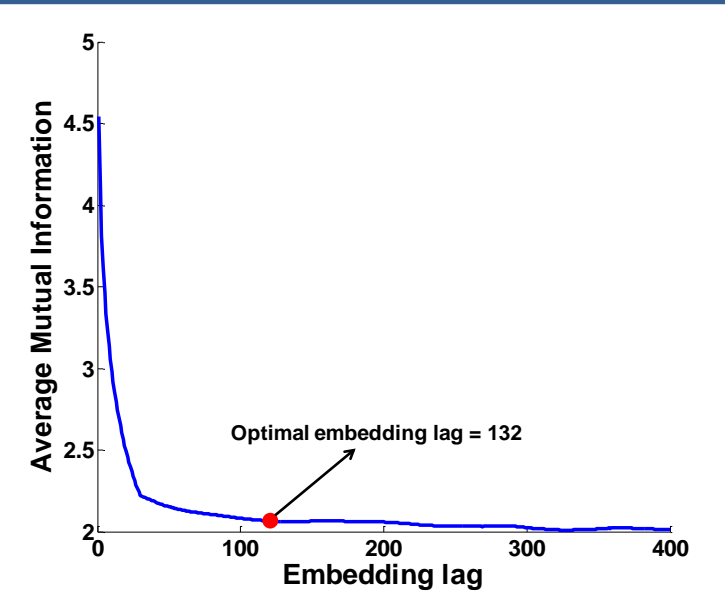

(b)

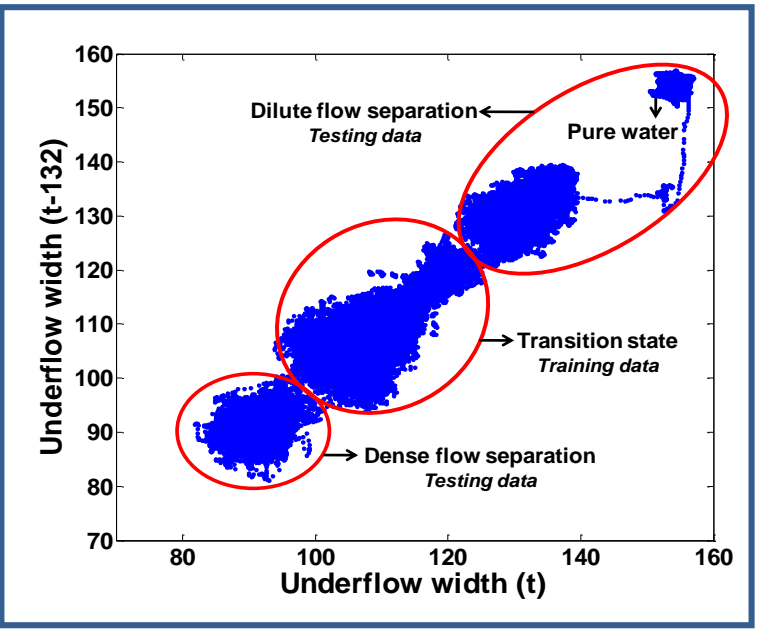

Figure 6-6: (a) Average Mutual Information plot and (b) resultant phase space for Gold case study

The representation of operating states through clusters indicates that the system dynamics are successfully encapsulated by the phase space of the underflow width. Since the phase space captures all of the operating states, an embedding dimension of two also proves to be sufficient. Ultimately, these results justify the original choice of underflow width as entity to quantify the variation in underflow shape and also indicate that the video camera and image analysis technique are sufficiently sensitive.

For subsequent modelling, only the transition state data, which is considered normal operating conditions, was specified as training data (hence the description of one-class SVM as an unsupervised learning technique). Dilute and dense flow separation data, which represent fault conditions, subsequently served as testing data.

\subsubsection{Optimization of kernel bandwidth, $\gamma$}

Results for the tested kernel bandwidths, ranging from $2^{-19}$ to $2^{9}$, are illustrated in figure 6-7. The resultant decision boundaries are indicated as the coloured lines.

These results show that with a decrease in $\gamma$-value, the decision boundary becomes more circular and loose-fitting. The class region is hereby extended beyond its actual boundaries, increasing the chances of misclassification. On the other hand, an increase in $\gamma$-value results in increased number of support vectors and a tighter, more irregular decision boundary. If the $\gamma$-value is too high, for instance $2^{9}$, the boundary is overfitted. 


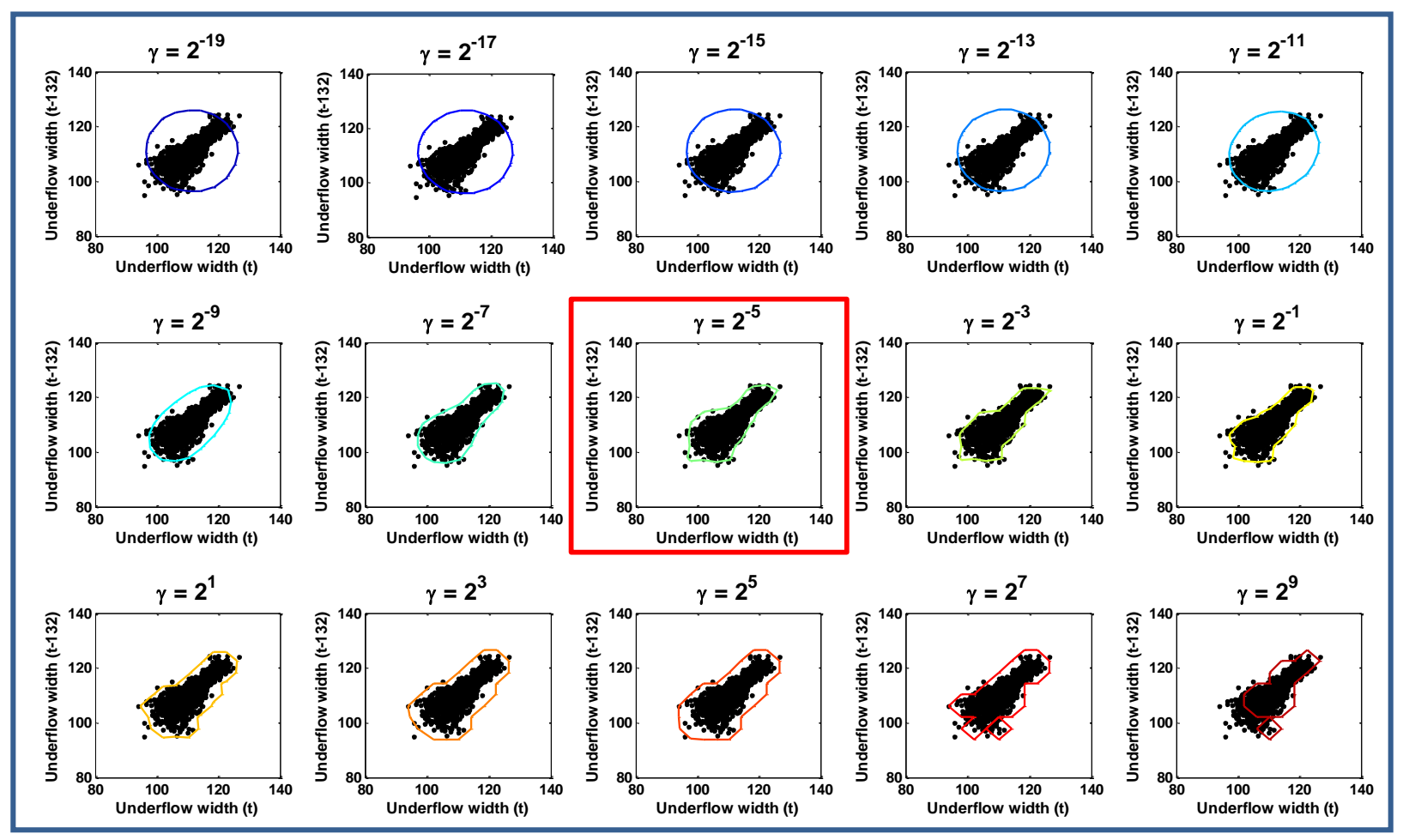

Figure 6-7: Optimization of kernel bandwidth for Gold case study

As depicted in figure 6-7, a value of $2^{-5}$ was selected for the Gold case study. Optimal $\gamma$ values for the other case studies were selected in a similar fashion and are summarized in appendix D-2. These values served as input to establish the final one-class SVM classification models, as presented in the following section.

\subsubsection{One-class SVM classification results}

\section{(a) Gold case study}

By employing the automatically selected kernel bandwidth, the one-class SVM model was retrained on the training data. Every $50^{\text {th }}$ point was sub-sampled for this purpose (this convention was used in all case studies). Figure 6-8 provides the final classification results.

To simplify interpretation, the general traits of the one-class SVM classification results are briefly described. The decision boundary of the training data (or transition state) is represented by the white line in figure 6-8. Any points that are predicted to fall outside this boundary, is recognized as fault conditions, implying that it does not belong to the transition state, but rather to dilute and dense flow separation. To account for outliers, a limit was specified on the decision boundary as the 99 percentile of the transition state. 


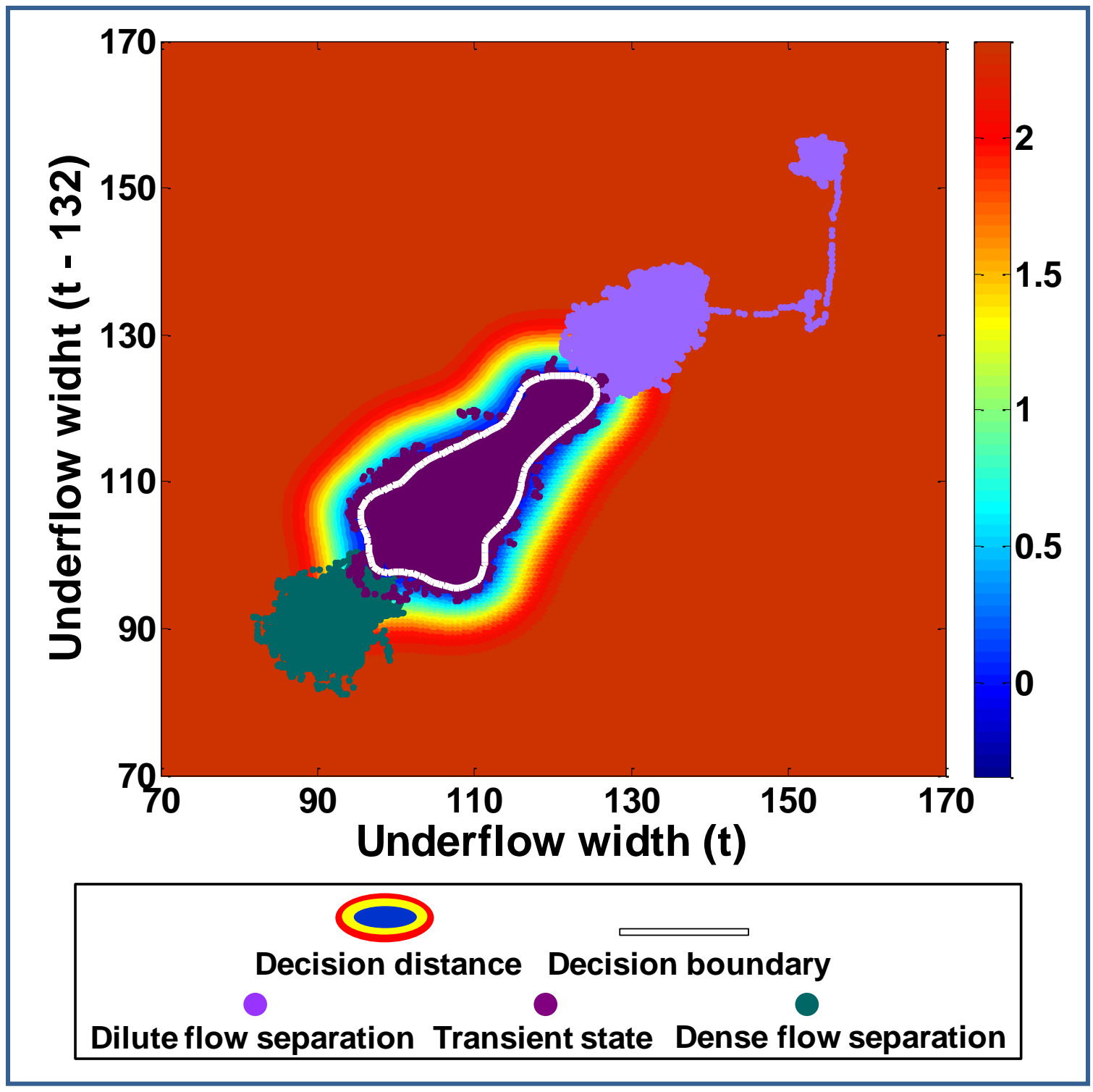

Figure 6-8: One-class SVM classification results for Gold case study

Colour contours furthermore give an indication of the decision distance. The decision distance is a distance measure from the densest region in the training data. With regards to the figure, the training data was specified as negative values (dark blue region). The more positive (or more red) a region becomes, the further away it is situated from the densest region in the training data*.

Inspection of figure 6-8 reveals that the decision boundary successfully excludes the fault conditions (dilute and dense flow separation). In order to measure the accuracy more specifically, false alarm rates (FAR's) and missing alarm rates (MAR's) were specified as performance indicators. The FAR refers to the percentage of points which is erroneously

\footnotetext{
* In the original one-class SVM model, the colour field was inverted with positive values corresponding to the data within the decision boundary and negative values for data outside the boundary. The inversion of this colour field was simply a matter of convenience (in order for red areas to represent fault conditions).
} 
classified as fault conditions, while the MAR is the percentage of points mistakenly recognized as part of the transition state.

For the Gold case study, the FAR is $1.00 \%$ (refer to table 6-2). This is expected, since the FAR was directly enforced through the 99 percentile limit on the training data. Dilute and dense flow separation have MAR's of $0.00 \%$ and $0.023 \%$ respectively. The extremely low MAR's indicate that one-class SVM is very accurate in predicting to which class a point belongs for this case study.

\section{(b) Platreef case study}

The Platreef case study differs from the other case studies since it consists of transition state data, followed by a short period of blocking, another short transition state and a final stage of blocking (refer to figure 6-5 (b)). For the purpose of one-class SVM classification, only the first transition state data was specified as training data, while the remaining data served as testing (unseen) data.

Classification through one-class SVM resulted in a $1.00 \%$ FAR for the first transition state (as predetermined through the application of the 99 percentile) and $1.28 \%$ FAR for the second transition data (refer to figure 6-9 (a) and table 6-2). A MAR of $6.06 \%$ is furthermore associated with the first blocking stage, while the MAR for the final blocking stage is $0.00 \%$.

These results are regarded as fairly good, considering the instability with which this hydrocyclone system operated. Results further indicate that the technique might be extended to detect instantaneous blocking (in comparison to a system that progresses from the transition state, to dense flow separation with roping discharge and finally a blockinglike discharge).

\section{(c) Merensky 1 case study}

In section 6.2.2 (a) it was found that the Merensky 2 case study displayed significant overlap between the underflow widths obtained for each operating state. The irregularities in the general downward trend were primarily caused by the large degree of foreground noise. The effect of the foreground noise further perpetuates to the final one-class SVM classification results. Figure 6-9 (b) shows how the decision boundary adapts to a more irregular shape (compared to other cases studies) to account for the increased variation in the transient state. A large degree of misclassification also occurs with MAR's for dilute and dense flow separation of $31.9 \%$ and $15.2 \%$ respectively. These unsatisfactory monitoring results reconfirm the detrimental effects of foreground noise and the importance of minimizing its presence. 

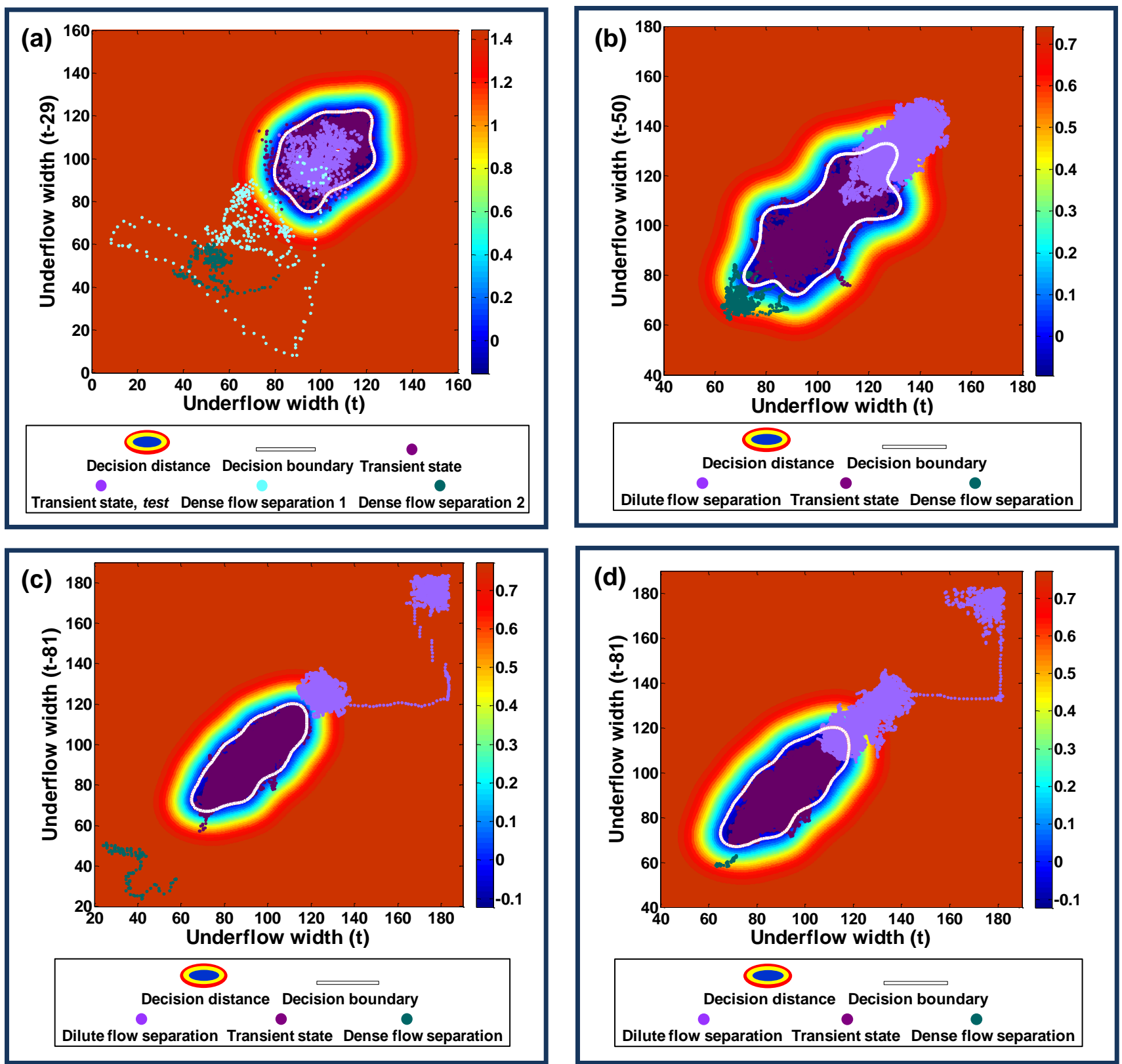

Figure 6-9: One-class SVM classification results for (a) Platreef, (b) Merensky 1, (c) Merensky 2 and (d) Merensky 3 case studies

Table 6-2: Performance indicators for one-class SVM classification

\begin{tabular}{|c|c|c|c|}
\hline \multirow{2}{*}{ Case study } & False alarm rate & Missing alarm rate & Missing alarm rate \\
\cline { 2 - 4 } & Transition state & Dilute flow separation & Dense flow separation \\
\hline Gold & $1.00 \%$ & $0.00 \%$ & $0.023 \%$ \\
\hline Platreef & Train: 1.00\%; Test: 1.28\% & N/A & $1: 6.06 \% ; 2: 0.00 \%$ \\
\hline Merensky 1 & $1.00 \%$ & $31.90 \%$ & $15.20 \%$ \\
\hline Merensky 2 & $1.00 \%$ & $0.00 \%$ & $0.00 \%$ \\
\hline Merensky 3 & $0.42 \%$ & $16.60 \%$ & $0.00 \%$ \\
\hline
\end{tabular}




\section{(d) Merensky 2 and 3 case study}

Although one-class SVM performs well with the unseen test data provided so far, the true test is to determine its prediction capabilities for a complete unseen data set. The duplicate case studies, Merensky 2 and 3, provide this opportunity. Model training was performed on the transitions state of the Merensky 2 case study. The dilute and dense flow separation data of the same case study consequently served as test data, as well as the whole Merensky 3 case study. Figure 6-9 (c) and (d) provides the respective classification results.

For the Merensky 2 case study, the one-class SVM prediction is $100 \%$ accurate and all fault conditions are successfully excluded from the transition state (refer to table 6-2). This is manifested by the MAR's of $0.00 \%$ for both dilute and dense flow separation. By using the same training data and testing it on the entire Merensky 3 case study, it was found that $16.6 \%$ of dilute flow separation was erroneously classified as part of the transition state. However, all points belonging to dense flow separation were accurately recognized as faults conditions (MAR of $0.00 \%$ ). In terms of detecting deviations from the transient state, the recognition of dense flow separation is considered far more important than dilute flow separation. In light of this, the latter results show that the application of one-class SVM to a completely unseen data set is very successful.

\subsubsection{Comparison of one-class SVM and multivariate Gaussian distribution (MVGD)}

To investigate the true value added by one-class SVM, a brief comparison is made to a much simpler classification technique, MVGD. The MVGD is simply an extension of the normal univariate distribution to higher dimensions. The mathematical definition of MVGD probability density function is provided in appendix C-3.

As example, one-class SVM and MVGD were both applied to the Gold case study, and the final results are presented in figure 6-10 (a) and (b) and table 6-3. Unlike the one-class SVM results, the coloured contours of the MVGD results do not give an indication of the decision distance, but rather of the probability density. The probability density describes the probability with which a point will occur in a specific region. To match the colour contours of the one-class SVM results, the probability density values of MVGD were assigned negative values.

The results show how the ellipsoidal decision boundary of MVGD underfits the transition state data. Consequently, the degree of misclassification is much higher for MVGD than for one-class SVM. This is validated through the high MAR's achieved for MVGD: $16.00 \%$ and $15.60 \%$ for dilute and dense flow separation respectively. In comparison, one-class SVM 
performs exceptionally well. These results reaffirm the use of a computationally more expensive and more robust technique, such as one-class SVM.
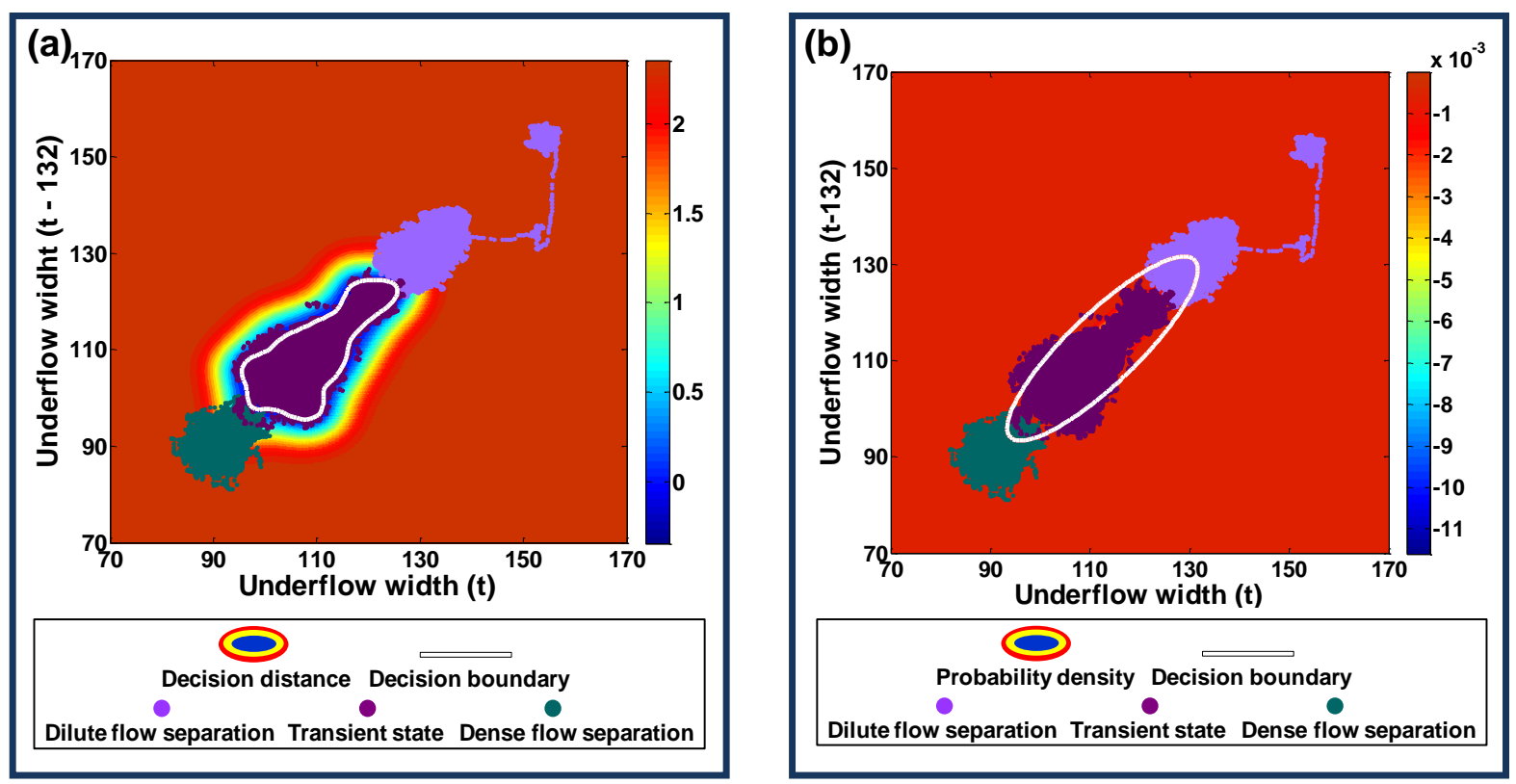

Figure 6-10: Comparison of (a) SVM and (b) MVGD classification results for Gold case study

Table 6-3: Comparison of performance indicators for SVM and MVGD results for Gold case study

\begin{tabular}{|c|c|c|c|}
\hline \multirow{2}{*}{$\begin{array}{c}\text { Classification } \\
\text { technique }\end{array}$} & False alarm rate & Missing alarm rate & Missing alarm rate \\
\cline { 2 - 4 } & Transition state & Dilute flow separation & Dense flow separation \\
\hline SVM & $1.00 \%$ & $0.00 \%$ & $0.023 \%$ \\
\hline MVGD & $1.00 \%$ & $16.00 \%$ & $15.60 \%$ \\
\hline
\end{tabular}

\subsubsection{Sensitivity of one-class SVM to embedding lag}

In practical terms, the embedding lag relates to the historical data required in the underflow width time series for the detection of a certain operating state. For example, for the Gold case study the optimal embedding lag is 132 , which relates to a sampling time of $5.4 \mathrm{~s}$. This implies that the combination of the current data point, and a point $5.4 \mathrm{~s}$ in the past, can be used to identify the current operating state. A summary of the optimal lag values, and the corresponding sampling times, is provided in appendix D-2.

The lag values incorporated in the one-class SVM classification were the optimal lag values as determined by AMI. These lag values were determined based on complete case studies, spanning from dilute to dense flow separation. In reality though, the calibration data which will be used to determine the lag values, would typically only contain normal operating data from the transition state (since fault conditions such as dense flow separation would be avoided). The question that arises is whether a lag can be determined from such an 
abbreviated data set to accurately capture the system dynamics. To shed light on this question, different lag values were specified for the Merensky 2 case study and the results investigated.

Phase spaces were generated for three different lag values: the optimal lag $81,30^{*}$ and 130 . By comparing the results, illustrated in figure 6-11 (a) to (c), it is found that the optimal lag assures an optimal distribution between the operating states in the phase space, as expected. For lag values other than the optimum, minor distortion of the phase space occurs. It is noticed however, that dense flow separation continually presents as a distinctly different cluster. Considering the robustness of one-class SVM, it is expected that dense flow separation will always be recognized separately from the transition state. The MAR's, which remain unchanged for all three lag values $(0.00 \%$ for both dilute and dense flow separation) confirms that this is in fact the case.

(a)

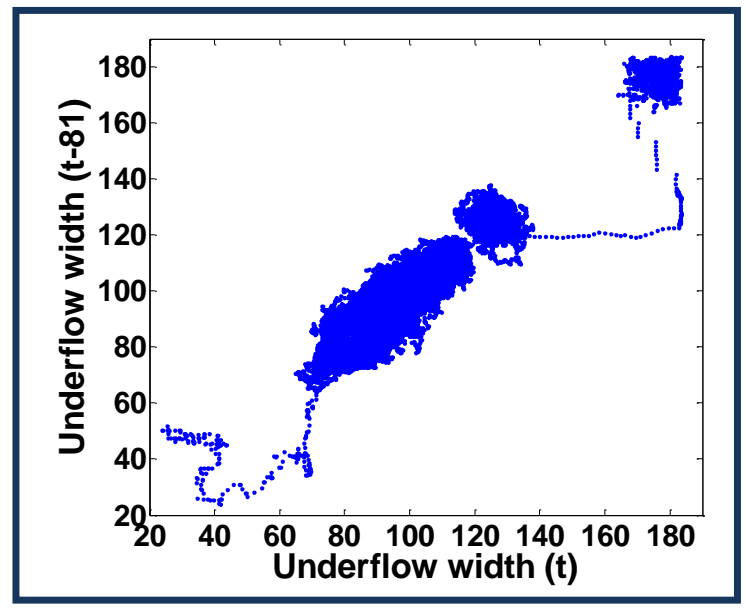

(b)

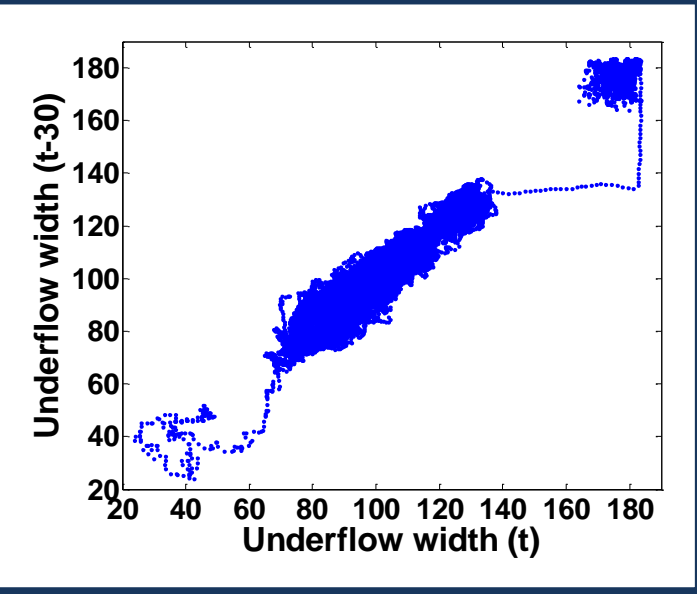

(c)

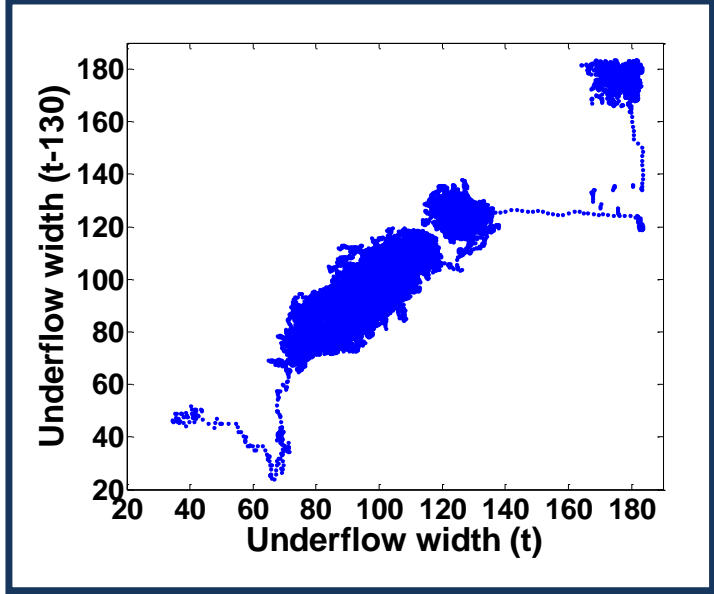

Figure 6-11: Phase space of Merensky 2 case study for embedding lags of (a) 81, (b) 30 and (c) 130

\footnotetext{
* For any relatively short set of training data, the default lag will be 29 due to the applied moving average of every 30 points. Lag values, below 29, are therefore not expected.
} 
These findings suggest that the one-class SVM results are not sensitive to the specified changes in the lag value, since the changes in underflow width between different operating states are sufficiently distinct. For a system with less distinct changes in underflow width though (as with the Gold case study due to slightly lower contrast differences), there might be a slight increase in sensitivity. A thorough investigation of the embedding lag sensitivity is therefore recommended. In the interim, it is suggested that the monitoring system should be recalibrated regularly in an industrial environment, as general good practice. Not only might the lag be readjusted during such a process, but any changes in contrast difference and lighting conditions can be taken into account by updating user inputs. The next logical step in the investigation will therefore also be to perform tests on an industrial hydrocyclone, which is continually subjected to varying feed and operating conditions.

\subsection{Final comments on monitoring system}

The following discussion details a few final comments regarding the image analysis and diagnostic monitoring techniques.

\subsubsection{Applicability to different ore types}

In terms of image analysis, the major difference between the different ore types is colour. As previously stated, the ore colour affects the slurry colour and consequently the contrast difference. If the contrast difference is too low, the developed image analysis technique proves ineffective (as with the IImenite case study). Other differences in ore properties, such as density and PSD's, did not affect the applicability of the image analysis or diagnostic monitoring technique.

\subsubsection{Operability of monitoring system}

In general, the operability of the monitoring system is not considered too complex. Some user subjectivity is introduced with the specification of contrast and brightness adjustments as well as the motion threshold. A general rule of thumb is to increase the interpretability of the underflow to the computing system by making the underflow stream optimally discernable to a human observer. The specification of the extracted horizontal line is also deemed simple with the knowledge of the underflow spray profile.

Parameter specification for one-class SVM classification normally requires some expert knowledge. This process has been simplified, though, by incorporating automated parameter selection. Some expert knowledge is still required for the specification of representative transition state data, for the purpose of calibration. Firstly, the training data should consist of enough points. This is not a problem, however, since a large amount of data is generated very quickly. Furthermore, the training data should also represent a fair 
degree of variation within the transition state itself. It should not be necessary to force the system too close to dense flow separation though, since this state is distinctly different from the transition state. Considering the aforementioned, it is thought that a plant-operator, familiar with hydrocyclone setups, should be able to effectively calibrate such a system.

\subsubsection{Processing time}

Although one-class SVM might be regarded as a computationally expensive technique, process time might be reduced significantly by sub-sampling the training data. In this study, the sub-sampling of every $50^{\text {th }}$ point proved very effective in this regard. Generally, smoother and better fitting decision boundaries are also obtained for sub-sampled cases.

The processing time of overall monitoring technique (image analysis and diagnostic monitoring) is considered sufficient in terms of on-line monitoring, since it is approximated as $1 \mathrm{~s}$ for every 30 video frames (or $1 \mathrm{~s}$ per $1 \mathrm{~s}$ of video data). By increasing computer power, or opting for parallelization, the processing time required for output can be minimized even further. 


\section{CHAPTER 7: CONCLUUSIONS AND RECOMIMIDNDATIONS}

In the following chapter, the conclusions are presented. Special emphasis is placed on how these conclusions relate to the objectives of the study. The discussion subsequently ends with recommendations made to improve various aspects of the developed monitoring technique.

\subsection{Conclusions}

As reminder, the secondary objectives stated in chapter 1 are reiterated and presented with the related conclusions:

- Perform a literature review of sensing techniques which have previously been employed and evaluate the use of image analysis by comparing its advantages and disadvantages to that of previous techniques.

The literature review presented in chapter 3 reveals that various sensing techniques have been applied in the past, with varying degrees of success. Widespread adoption of these techniques has been limited though, since most techniques do not comply with the requirements of the hydrocyclone monitoring system (as stipulated by Neesse, Schneider et al., 2004). General technique application is further hindered by the complexity of the methods, the extensive alterations required and the need for regular maintenance.

Based on the literature survey, image analysis of the underflow proved a viable option, since two previous studies incorporating the method demonstrated compliance with the hydrocyclone monitoring requirements (Petersen et al, 1996; Neesse, Schneider et al., 2004). As additional advantage, image analysis would furthermore provide the opportunity for visual observation of the underflow at any time. This in turn, might simplify troubleshooting.

- Develop an image analysis technique which provides a satisfactory account of variation in underflow shape.

To account for the variation in underflow shape, it was decided to extract the underflow width during image analysis. An image analysis technique, based on motion detection and various noise reduction methods, was consequently developed to extract the underflow width. By comparing the final underflow width results with visual observations, it is concluded that the technique provides a very good account of underflow shape variation. 
Subsequent time series embedding and machine learning also confirms this, since clusters of the various operating states can be identified which encapsulate the system dynamics.

Only two exceptions (Ilmenite and Merensky 1 case studies) were identified to yield unsatisfactory results, as clarified in the next section.

- Investigate how image quality affects the image analysis process and determine to what extent images can be improved, if necessary.

The factors that were identified to affect the interpretability of the underflow to the computing system are contrast difference, background noise, foreground noise, random noise and system vibration. Image enhancement and the application of a motion threshold proved very effective in the reduction of background noise, random noise and system vibration.

Although contrast and brightness adjustment can also be used to increase the contrast difference to a large extent, there is a limit to this specific application. If there is almost no existing contrast difference between the underflow and surrounding environment, manipulation through contrast and brightness is useless (as with the Ilmenite case study). To minimize foreground noise, underflow recognition was restricted to ISL, with the supposition that foreground noise will fall outside these limits. For cases with extreme amounts of foreground noise, this technique is ineffective in the removal of noise overlapping with the underflow edge. This leads to the false exaggeration of underflow widths, as with the Merensky 1 case study.

Considering that edge detection is a non-trivial technique which might similarly be susceptible to the mentioned types of image noise, the original choice for motion detection as feature extraction technique is reaffirmed.

- Determine whether underflow data, obtained through image analysis, can be used to identify the various operating states, in specific the troublesome dense flow separation.

By comparing image analysis results to visual observations, it is noticed that the changeover between operating states are marked by decreases in underflow width. The operating states can furthermore be identified as separate clusters in the phase space. Ultimately, any deviations from the transition state can automatically be recognized through application of one-class SVM, by simply providing examples of the transition state as training data. Dense flow separation, in specific, was classified with extremely high accuracy (except for the Merensky 1 case study due to foreground noise). The true value of this technique was further illustrated by the successful classification ( $0.00 \% \mathrm{MAR})$ of dense flow separation of a completely unseen data set, based on the training data of a duplicate case study. 
In terms of classification, a brief comparison showed that MVGD performs very poorly compared to one-class SVM. These results served as additional justification for the application of the computationally more expensive technique, one-class SVM. Preliminary results from the Gold case study also suggest that one-class SVM results are not sensitive to the embedding lag.

\section{- Investigate the applicability of the technique to different ore types.}

The four different ore types that were included in the study are gold, ilmenite and PGM ore from both the Platreef and Merensky reef. In terms of image analysis, a prominent difference between these four ore types is ore colour. The ore colour determines the slurry colour, which consequently influences the contrast difference between the underflow stream and surrounding environment. For the ilmenite case study, the dark ore colour against the dark surrounding environment, proved detrimental in terms of image analysis.

Except for the aforementioned case, the image analysis and diagnostic monitoring techniques were successfully applied to the other different ore types, in spite of different ore properties.

\section{- Evaluate the practicality of the developed monitoring system with reference to the hydrocyclone monitoring requirements set by Neesse, Schneider et al. (2004).}

To recap, the hydrocyclone monitoring requirements as stipulated by Neesse, Schnieder et al. (2004) are: sensitivity, non-invasiveness, sampling times smaller than $1 \mathrm{~s}$, robustness and cost-effective equipment.

The fact that the underflow width results compare well to visual observations, serves as indication that the sensitivity of the monitoring system is sufficient to account for variation in the underflow shape. Since the monitoring system consists of a video camera, which can record the underflow from a distance, the technique proves to be completely non-invasive to hydrocyclone operation. Due to the fast shutter speed of the video camera (30 frames per second), underflow images are also sampled at a sufficient rate. An approximate processing to video time ratio of $1: 1$, is also very promising. For cases with prominent foreground noise, the monitoring system lacks robustness though. However, since foreground noise can be easily eliminated through simple physical alterations (refer to recommendations), the general robustness is considered satisfactory. All equipment are also mass-produced consumer products and therefore relatively inexpensive.

Additionally, the installation of the monitoring system does not require extensive alterations to existing hydrocyclone setups. The technique is furthermore not considered maintenance intensive. In terms of ease of use, the application of the monitoring system is considered 
relatively straightforward. Even though one-class SVM application is complex in general, the need for expert input has been minimized through the automation of parameter selection. The only expert knowledge required is related to the provision of a typical set of training data, a task that might easily be performed by an operator familiar with the process.

Finally, the primary objective is presented, together with the relevant conclusions:

\section{- Explore the feasibility of image analysis of hydrocyclone underflow as a monitoring technique.}

The results of this study show that image analysis of hydrocyclone underflow can be regarded as a viable monitoring technique. This statement is supported by the extremely accurate identification of operating states (in specific the troublesome dense flow separation) as well as the overall practicality of the developed system. Existing shortcomings, as experienced with the Ilmenite and Merensky 1 case studies, might be addressed by adjustments to the physical setup (refer to recommendations).

\subsection{Recommendations}

It is foreseen that image quality might be improved during the recording process by making certain changes to the physical monitoring setup. By directing a light towards the underflow, for instance, improved contrast difference can be obtained due to the reflective properties of the underflow stream. The use of ample lighting also aids in the reduction of random noise.

Alternatively, backlighting might be used to create a silhouette image of the underflow. Conceptually, backlighting will effectively ensure sufficient contrast between the underflow and surroundings. The success of the technique will therefore no longer depend on the ore colour. Backlighting would furthermore also assist in the elimination of background noise. Since the concept of backlighting remains untested in the current study, though, it is recommended that the effect thereof on image quality should be investigated thoroughly.

To eliminate foreground noise, the video camera should be positioned outside the hydrocyclone splash zone. If this is not possible due to spatial limitations, an air-knife might prove useful in this regard. By fixing a video camera to a stationary system, the prominence of system vibration in video footage would also be minimized.

In terms of the image analysis technique itself, refinements might be made by investigating other noise reduction methods such as filtering and thresholding. Regarding the diagnostic monitoring, a more thorough investigation of the sensitivity to the embedding lag might be performed. 
Ultimately, future work should focus on testing the monitoring technique on an industrial hydrocyclone setup. The system would hereby be subjected to unforeseen changes in operating states (in comparison to the manually induced changes in operating state during this study). To further account for changes in lighting and contrast conditions, regular system calibrations are recommended for such industrial applications. 


\section{REFERENCES}

Acton, S.T. and Mukherjee, D.P., 2000. Area operators for edge detection. Pattern Recognition Letters, 21(8), 771-777.

Barrientos, A. and Concha, F., 1992. Phenomenological model of classification in conventional hydrocyclones. In Comminution - Theory and Practice. Littleton: SME Inc., pp. 287-305.

Braun, T. and Bohnet, M., 1990. Influence of feed solids concentration on the performance of hydrocyclones. Chemical Engineering and Technology, 13(1), 15-20.

Castro, O., 1990. Pulp Rheology Effects for Hydrocyclone Models, M.Sc. Thesis, Brisbane, Australia: University of Queensland (JKMRC).

Castro, O., Concha, F., Montero, J., Miranda, J., Castro, J. and Urizer, D., 1996. Air core modelling for an industrial hydrocyclone. In Hydrocyclones 96. London: Mechanical Engineering Publications Limited, pp. 229-240.

Chang, C. and Lin, C., 2001. LIBSVM - A Library for Support Vector Machines. Available at: http://www.csie.ntu.edu.tw/ cjlin/libsvm [Accessed November 4, 2010]

Coulson, J.M., Richardson, J.F., Backhurst, J.R. and Harker, J.H., 1991. Chemical Engineering vol 4, Oxford: Butterworth Heinemann.

Cristea, E.D., Malfa, E. and Coghe, A., 1994. 3D Simulation and experiments of cement rotary kiln preheater top cyclone. In Proceedings of Fluent European User's Group Meeting. Harrogate, p. C54.

Dlamini, M.F., Powell, M.S. and Meyer, C.J., 2005. A CFD simulation of a single phase hydrocyclone flow field. Journal of the South African Institute of Mining and Metallurgy, $105,711-717$

Dombrowski, N. and Hasson, D., 1969. The flow characteristics of swirl (centrifugal) spray pressure nozzles with low viscosity liquids. American Institute of Chemical Engineers Journal, 15(4), 604-611.

Dyakowski, T. and Williams, R.A., 1993. Modelling turbulent flow within a small-diameter hydrocyclone. Chemical Engineering Science, 48(6), 1143-1152. 
Dyakowski, T., Jeanmeure, L. and Jaworski, A., 2000. Applications of electrical tomography for gas-solids and liquid-solids flows - a review. Powder Technology, 112(3), 174-192.

Elsner, J.B. and Tsonis, A.A., 1996. Singular Spectrum Analysis - A new tool in time series analysis, New York: Plenum Press.

Fraser, A.M. and Swinney, H., 1986. Independent coordinates for strange attractors from mutual information. Physical Review A, 33, 1134-1140.

Galvin, K.P. and Smitham, J.B., 1994. Use of X-rays to determine the distribution of particles in an operating cyclone. Minerals Engineering, 7(10), 1269-1280.

Gonzalez, R.C. and Woods, R.E., 2008. Digital Image Processing $3^{\text {rd }}$ ed., Upper Saddle River: Prentice Hall

Gupta, R., Kaulaskar, M.D., Kumar, V., Sripriya, R., Meikap, B.C. and Chakraborty, S., 2008. Studies on the understanding mechanism of air core and vortex formation in a hydrocyclone. Chemical Engineering Journal, 144(2), 153-166.

Gutiérrez, J.A., Dyakowski, T., Beck, M.S. and Williams, R.A., 2000. Using electrical impedance tomography for controlling hydrocyclone underflow discharge. Powder Technology, 108(2-3), 180-184.

Hou, R., Hunt, A. and Williams, R.A., 1998. Acoustic monitoring of hydrocyclone performance. Minerals Engineering, 11(11), 1047-1059.

Hou, R., Hunt, A. and Williams, R.A., 2002. Acoustic monitoring of hydrocyclones. Powder Technology, 124(3), 176-187.

Hsieh, K.T. and Rajamani, K., 1988. Phenomenological model of the hydrocyclone: Model development and verification for single-phase flow. International Journal of Mineral Processing, 22(1-4), 223-237.

Hsieh, K.T. and Rajamani, R.K., 1991. Mathematical model of the hydrocyclone based on physics of fluid flow. American Institute of Chemical Engineers Journal, 37(5), 735-746.

Hulbert, D.G., 1993. Measurement Method and Apparatus for Hydrocyclones. Pat EP0522215A2, Randburg.

Hulbert, D.G., Personal communication, 19 August 2010. 
Industrial Tomography Systems plc., 2010. Industrial Tomography Systems. Electrical Resistance Tomography. Available at:

http://www.itoms.com/electrical_resistance_tomography_p2000_instrument_system_er $t$ _its [Accessed August 26, 2010].

Kawatra, S., 1996. The effect of slurry viscosity on hydrocyclone classification. International Journal of Mineral Processing, 48(1-2), 39-50.

Kawatra, S., Eisele, T.C., Zhang, D. and Rusesky, M., 1988. Effects of temperature on hydrocyclone efficiency. International Journal of Mineral Processing, 23(3-4), 205-211.

Kelly, E.G. and Spottiswood, D.J., 1982. Introduction to Mineral Processing, New York: John Wiley and Sons, Inc.

Lynch, A.J. and Rao, T.C., 1968. Studies on the operating characteristics of hydrocyclone classifiers. Indian Journal of Technology, 6(4), 106-114.

Lynch, A.J. and Rao, T.C., 1975. Modelling and scale-up of hydrocyclone classifiers. In Proceedings of 11th International Mineral Processing Congress. Cagliari, pp. 245-269.

Maini, R. and Aggarwal, H., 2010. A comprehensive review of image enhancement techniques. Journal of Computing, 2(3), 8-13.

Manabe, Y. and Chakraborty, B., 2007. Improved estimation of embedding parameters of nonlinear time series by structural learning of neural network with fuzzy regularizer. Journal of Advanced Computational Intelligence and Intelligent Informatics. 11(6), 600609.

Marlow, D., 1973. A Mathematical Analysis of Hydrocyclone Data, M.Sc. Thesis, Brisbane, Australia: University of Queensland (JKMRC).

McHugh, S., 2010. Cambridge in Colour. Understanding Image Noise. Available at: http://www.cambridgeincolour.com/tutorials/image-noise.htm [Accessed October 11, 2010]

Mesnik, B., 2004. Kintronics, Inc. Understanding Camera Specifications. Available at: http://www.kintronics.com/neteye/neteye.html [Accessed October 05, 2010]

Mintek, 1992. Technology transfer awards. Journal of the South African Institute of Mining and Metallurgy, 92(10), 299. 
Monredon, T.C., Hsieh, K.T. and Rajamani, R.K., 1992. Fluid flow model of the hydrocyclone: an investigation of device dimensions. International Journal of Mineral Processing, 35(12), 65-83.

Nageswararao, K., 1978. Further Developments in the Modelling and Scale-up of Industrial Hydrocyclones, Ph.D. Thesis, Brisbane, Australia: University of Queensland (JKMRC).

Nageswararao, K., Wiseman, D.M. and Napier-Munn, T., 2004. Two empirical hydrocyclone models revisited. Minerals Engineering, 17(5), 671-687.

Napier-Munn, T.J., Morrel, S., Morrrison, R.D. and Kojovic, T., 1996. Mineral Comminution Circuits - Their Operation and Optimzation, Indooroopilly: University of Queensland (JKMRC).

Narasimha, M., Sripriya, R. and Banerjee, P.K., 2005. CFD modelling of hydrocyclone-prediction of cut size. International Journal of Mineral Processing, 75(1-2), 53-68.

Neesse, T., Dallmann, W. and Epsig, D., 1986. The influence of turbulence on the separation result of hydrocyclones at high feed solids contents. Aufbereitungstechnik, 27, 6-14.

Neesse, T. and Dueck, J., 2007. Air core formation in the hydrocyclone. Minerals Engineering, 20(4), 349-354.

Neesse, T., Golyk, V., Kaniut, P. and Reinsch, V., 2004. Hydrocyclone control in grinding circuits. Minerals Engineering, 17(11-12), 1237-1240.

Neesse, T., Schneider, M., Golyk, V. and Tiefel, H., 2004. Measuring the operating state of the hydrocyclone. Minerals Engineering, 17(5), 697-703.

Neesse, T., Schubert, H. and Graichen, K., 1991. Practical and theoretical aspects of denseflow separation. Aufbereitungstechnik, 32(9), 459-472.

Nowakowski, A.F., Cullivan, J.C., Williams, R.A. and Dyakowski, T., 2004. Application of CFD to modelling of the flow in hydrocyclones. Is this a realizable option or still a research challenge? Minerals Engineering, 17(5), 661-669.

Olson, T.J. and Turner, P.A., 2002 . Hydrocyclone selection for plant design. , In Proceedings of Mineral Processing Plant Design, Practice and Control, Littleton: SME Inc., pp. 880-893.

Olson, T. and Waterman, R., 2006. Hydrocyclone Roping Detector and Method. Pat US6983850B2, Tuscon. 
Petersen, K.R.P., Aldrich, C., Van Deventer, J.S.J., Mclnnes, C. and Stange, W.W., 1996. Hydrocyclone underflow monitoring using image processing methods. Minerals Engineering, 9(3), 301-315.

Plitt, L.R., 1976. Mathematical model of the hydrocyclone classifier. CIM Bulletin, 69(776), 114-123.

Possa, M. and Lima, J., 2000. The effect of viscosity on small-diameter hydrocyclones' performance in desliming process. In Proceedings of the 21st International Mineral Processing Congress. Rome: Elsevier Science, pp. C4:29-35.

Ruelle, D., 1980. Measures describing turbulent flow. Annals of New York Academy of Science, 357(1). 1-9.

Russ, J.C., 1995. The Image Processing Handbook $2^{\text {nd }}$ ed., Boca Raton: CRC Press.

Schlaberg, H.I., Podd, F.J.W. and Hoyle, B.S., 2000. Ultrasound process tomography system for hydrocyclones. Ultrasonics, 38(1-8), 813-816.

Schölkopf, B., Platt, J., Shawe-Taylor, J., Smola, A.J. and Williamson, R.C., 2001. Estimating the support of a high-dimensional distribution. Neural Computation. 13, 1443-1471.

Schubert, H. and Neesse, T., 1980. A hydrocyclcone separation model in consideration of the turbulent multi-phase flow. In Proceedings International Conference on Hydrocyclones. Cambridge: BHRA Fluid Engineering, pp. 23-36.

Schubert, H. and Neesse, T., 1973. The role of turbulence in wet classification. In Proceeding of 10th International Mineral Processing Congress. Cannes: The Institute of Mining and Metallurgy.

Schweitzer, K., 1972. Hydrozyklon mit Selbstregelnder Unterlaufdüse. Pat DEGM7201703, Hochdal.

Shafie, A.A., Hafiz, F. and Ali, M.H., 2009. Motion detection techniques using optical flow. World Academy of Science, Engineering and Technology, 56, 559-561.

Shin, H.J., Eom, D. and Kim, S., 2005. One-class support vector machines - an application in machine fault detection and classification. Computers and Industrial Engineering, 48, 395408. 
Slack, M.D. and Wraith, A.E., 1997. Modelling the velocity distribution in a hydrocyclone. In 4th International Colloqium on Process Simulation. Espoo: Helsinki University of Technology, pp. 65-83.

Smith, S.W., 2003, Digital Signal Processing: A Practical Guide for Engineers and Scientists, Boston: Newnes.

Sripriya, R., Kaulaskar, M.D., Chakraborty, S. and Meikap, B.C., 2007. Studies on the performance of a hydrocyclone and modeling for flow characterization in presence and absence of air core. Chemical Engineering Science, 62(22), 6391-6402.

Statsoft Inc., 2010. Electronic Statistics Textbook. Available at:

http://www.statsoft.com/textbook/ [Accessed October 20, 2010].

Svarovsky, L., 1984. Hydrocyclones, Eastbourne: Holt, Rinehart and Winston Ltd.

Svarovsky, L., 2000. Solid-liquid Separation, Oxford: Butterworth-Heinemann.

Taggart, A.F., 1950. Handbook of Mineral Dressing, New York: John Wiley and Sons, Inc.

Takens, F., 1981. Detecting strange attractors in turbulence. Springer Lecture Notes in Mathematics, 898, 366-381.

Tarr, D.T., 1985. Hydrocyclones. In SME Mineral Processing Handbook. New York: Society of Mining Engineers of the American Institute of Mining, Metallurgical and Petroleum Engineers, pp. 3D1-3D59.

Tromans, D. and Meech, J.A., 2002. Impacting Energy Efficiency: Improving Energy Use in Grinding. Available at: http://www.mining.ubc.ca/cerm3/energy\%20efficiency.html [Accessed June 01, 2010]

Van Deventer, J.S.J., Feng, D., Petersen, K.R.P. and Aldrich, C., 2003. Modelling of hydrocyclone performance based on spray profile analysis. International Journal of Mineral Processing, 70(1-4), 183-203.

Wang, F., Marashdeh, Q., Fan, L.S. and Williams, R.A., 2009, Electrical capacitance, electrical resistance and positron emission tomography techniques and their applications in multiphase flow systems. In Advances in Chemical Engineering vol. 37. Amsterdam: Academic Press, pp. 179-222.

Wesseling, P., 2001. Principles of Computational Fluid Dynamics, Heidelberg: Springer. 
West, R., Jia, X. and Williams, R.A., 2000. Parametric modelling in industrial process tomography. Chemical Engineering Journal, 77(1-2), 31-36.

Wiliams, R.A., Dyakowski, T., Xie, C.G., Luke, S.P., Gregory, P.J., Edwards, R.B. and Gate, L.F., 1995. Industrial measurement and control of particulate processes using electrical tomography. In Process Tomography - Implementation for Industrial Processes. Manchester: University of Manchester, pp. 3-15.

Williams, R.A., Ilyas, O.M., Dyakowski, T., Dickin, F.J., Gutiérrez, J.A., Wang, M., Beck, M.S., Shah, C. and Rushton, A., 1995. Air core imaging in cyclonic separators: implications for separator design and modelling. The Chemical Engineering Journal, 56, 135-141.

Williams, R.A., Peng, S. and Brown, D., Parkinson, N. and Jannes, P., 1996. On-line measurement of hydrocyclone performance using acoustic emission. In Hydrocyclones 96. London: Mechanical Engineering Publications Limited, pp. 241-252.

Williams, R.A., Dickin, F.J., Gutiérrez, J.A., Dyakowski, T. and Beck, M.S., 1997. Using electrical impedance tomography for controlling hydrocyclone underflow discharge. Control Engineering Practice, 5(2), 253-256.

Williams, R.A., Jia, X., West, R.M., Wang, M., Cullivan, J.C., Bond, J., Faulks, I., Dyakowski, T., Wang, S.J., Climp;son, N., Kostuch, J.A. and Payton, D., 1999. Industrial monitoring of hydrocyclone operation using electrical resistance tomography. Minerals Engineering, 12(10), 1245-1252.

Wills, B., 2003. Mineral Processing Technology $6^{\text {th }}$ ed., Oxford: Butterworth Heinemann.

Yalcin, T., Kaukolin, E. and Byers, A., 2003. Axial inlet cyclone for mineral processing applications. Minerals Engineering, 16(12), 1375-1381. 


\section{APPENDIX A: NOMIENCLATURE}

Table I: Nomenclature list

\begin{tabular}{|l|l|}
\hline Symbol & \\
\hline$B$ & Original blue colour pixel value \\
\hline$d$ & Embedding dimension \\
\hline$F$ & Underflow image / frame \\
\hline$G$ & Original green colour pixel value \\
\hline$G S$ & Greyscale pixel value \\
\hline$I$ & Average mutual information \\
\hline$i$ & Vector index \\
\hline$j$ & Vector index \\
\hline$K$ & Kernel function \\
\hline$k$ & Image / frame index \\
\hline$m$ & Index corresponding to final member of vector \\
\hline$N$ & Index corresponding to final member of image data set \\
\hline$R$ & Original red colour pixel value \\
\hline$t$ & Time \\
\hline$u$ & Embedded underflow width vector \\
\hline$w$ & Underflow width \\
\hline$z$ & Decision boundary margin \\
\hline$\gamma$ & Kernel bandwidth \\
\hline$\varepsilon_{i}$ & Slack variable \\
\hline$v$ & Error limits \\
\hline$\rho$ & SVM threshold level \\
\hline$\tau$ & Embedding lag \\
\hline & Mapping function \\
\hline & Weight vector \\
\hline
\end{tabular}




\section{APPENDIX B: EXPERIMIENTAL DETAILS}

\section{B-1 Stepwise instructions for experimental procedure}

The following section provides stepwise instructions for the experimental procedure. These instructions are based on the general hydrocyclone setup as described in section 4.1. A schematic diagram of the setup is duplicated in figure I to provide quick reference for valve and equipment identification.

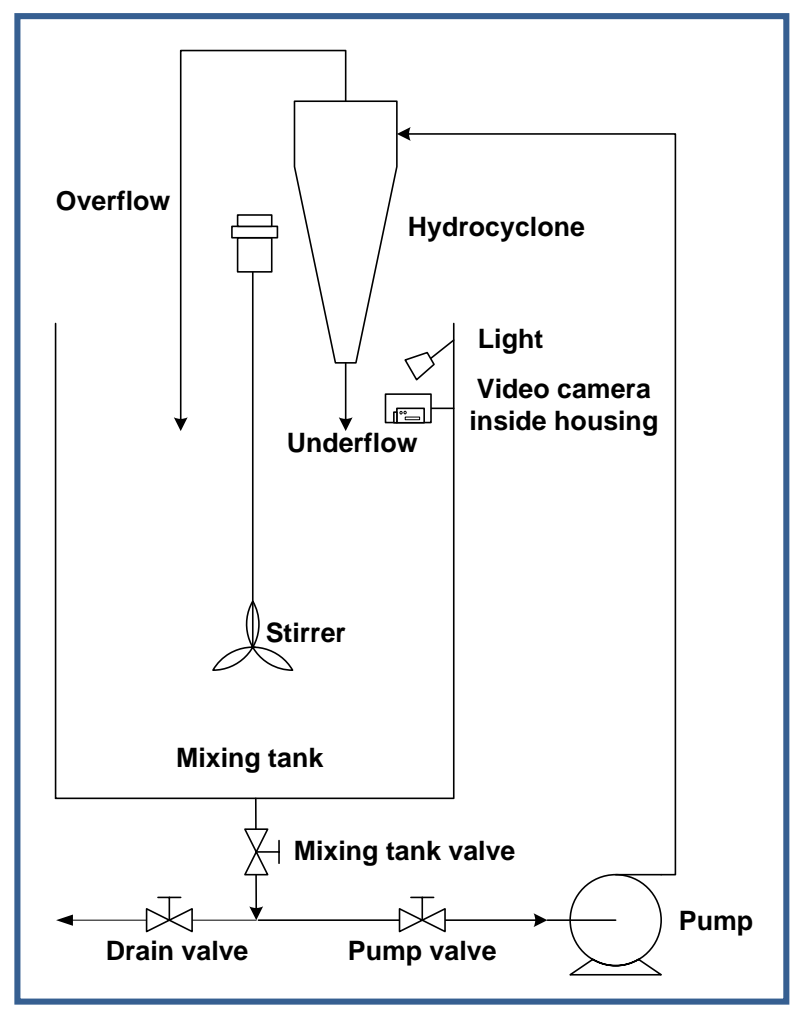

Figure I: Schematic of general hydrocyclone setup

\section{(a) Preparation}

The determination of the dry bulk ore PSD is executed before commencement of the experiment:

1. Collect a representative sample of the dry bulk ore by use of a rotary splitter.

2. Select the sieve trays to be used for the sieve analysis.

3. Pour the dry bulk ore sample through the selected sieve trays and fasten the trays to the vibrating sieve shaker.

4. Allow approximately 30 minutes for the sample to classify and switch off the sieve shaker. 
5. Loosen the sieves and determine the mass of ore left on each sieve tray.

The following steps describe the preparation of the experimental setup:

6. Direct the video camera housing towards the spigot opening and carefully place the video camera into it.

7. Connect the video camera to a power supply and switch it on.

8. To ensure that the recorded image is clearly visible, tilt the lens until the picture is in focus.

9. Direct the light towards the spigot and underflow region.

\section{(b) Start-up procedure}

1. Ensure that the mixing tank valve is closed before filling the tank with water.

2. Switch on the light and activate recording of the video camera.

3. Turn on the stirrer of the mixing tank. The stirring motion will aid in maintaining particle suspension in the water.

4. To allow the circulation of water through the system, close the drain valve, while opening the pump- and mixing tank valves. Switch the pump on immediately after this, to avoid the pump running dry.

\section{(c) Operating procedure}

1. Allow the water to circulate for 5 minutes in order to attain steady state.

2. Wipe the sightglass of the video camera housing with a clean, dry cloth. Cleaning the sightglass occasionally minimizes the amount of droplets, which splash against the window and obstruct the recorded image.

3. Weigh the first bucket of ore to be added to the system.

4. Each ore addition signifies the start of a new run. Indicate the start of each run by holding a sign, with the number of the run, at the spigot opening for a few seconds. The sign number helps to distinguish the different runs when editing the video.

5. Add the ore to the circulating fluid by slowly emptying the bucket into the mixing tank. Excessive splashing is minimised in this manner.

6. Allow another 5 minutes for the system to reach steady state.

7. Continually add ore to the system until roping conditions are accomplished. Repeat steps 2 to 6 with the start of each run.

8. During the course of the experiment, minimize physical movement on the structure on which the setup is mounted. Any movement on the structure causes excessive vibration of the hydrocyclone and the video camera. Consequently, the recorded image may become unsteady. 


\section{(d) Shut-down procedure}

1. On completion of the last run, stop video recording.

2. Disconnect all electrical equipment from the power supply.

3. Commence with cleaning the hydrocyclone system. While cleaning the system, the ore may settle in the piping and cause blockages. To reduce the likelihood of blockages, dilute the circulating fluid with water.

4. When the fluid is sufficiently dilute, start emptying the mixing tank by opening the drain valve. Continue to add water to the mixing tank.

5. Collect the fluid in a large container to allow the settling of the ore particles. This also permits the overflow of water to the floor drain.

6. Once the majority of particles are flushed from the system, switch off the pump.

7. Hose the remainder of the particles from the mixing tank. Close the mixing tank valve when the tank is completely clean and empty.

8. Flush the pump and piping system through the openings adjacent to the drain valve.

9. Also clean the pump sump.

10. Close the drain valve on completion of washing.

11. Clean the floor area of the experiment.

\section{B-2 Equipment specifications}

To give an indication of the size range of equipment, equipment specifications for one setup are provided in the following section:

- Dimensions of hydrocyclone

Spigot diameter $=32 \mathrm{~mm}$

Vortex finder diameter $=75 \mathrm{~mm}$

Upper body diameter $=158 \mathrm{~mm}$

Feed inlet diameter $=61 \mathrm{~mm}$

- Dimensions of mixing tank

Diameter $=750 \mathrm{~mm}$

Height $=1000 \mathrm{~mm}$

- Specifications of pump

Type: Warman $\mathrm{CH}$ gland seal pump

Power rating: $15 \mathrm{~kW}$ 


\section{B-3 Safety aspects}

The risks associated with the hydrocyclone systems that were used and accompanying experimental work are the following:

- injury due to moving machinery such as the mixing tank stirrer;

- electrical shock due to the presence of power cables in the vicinity of the hydrocyclone systems;

- damage to hearing because of loud noise associated with the hydrocyclone systems' operation;

- inhalation of dust particles during ore addition;

- danger of heights because of elevated hydrocyclone platforms;

- injury due to presence of overhead structures.

Special care was therefore taken to:

- prevent clothing or hair getting entangled in the mixing tank stirrer;

- keep power cables elevated and dry as far as possible;

- move cautiously on the elevated working platforms and keeping these surfaces as slipfree as possible;

- wearing the protective personal equipment (PPE) that aids in the prevention of hearing damage, dust inhalation and injuries to the head.

The following pieces of PPE were worn:

- laboratory coat;

- closed shoes;

- safety goggles;

- ear plugs;

- dust mask;

- safety helmet. 


\section{B-4 Experimental data: Sieve analysis results}

Table II: Sieve analysis results for gold ore

\begin{tabular}{|c|c|c|c|c|c|}
\hline \multirow{2}{*}{$\begin{array}{l}\text { Sieve size range } \\
(\mu \mathrm{m})\end{array}$} & \multicolumn{2}{|c|}{ Sieve fractions } & \multirow{2}{*}{$\begin{array}{c}\text { Nominal aperture size } \\
(\mu \mathrm{m})\end{array}$} & \multirow{2}{*}{ Cumulative \% undersize } & \multirow{2}{*}{ Cumulative \% oversize } \\
\hline & Weight (g) & Weight \% & & & \\
\hline+2360 & 0.41 & 0.41 & 2360 & 99.59 & 0.41 \\
\hline$-2360+1700$ & 0.90 & 0.90 & 1700 & 98.69 & 1.31 \\
\hline$-1700+1180$ & 1.66 & 1.66 & 1180 & 97.03 & 2.97 \\
\hline$-1180+850$ & 2.76 & 2.76 & 850 & 94.27 & 5.73 \\
\hline$-850+600$ & 4.47 & 4.47 & 600 & 89.80 & 10.20 \\
\hline$-600+425$ & 7.05 & 7.05 & 425 & 82.75 & 17.25 \\
\hline$-425+300$ & 11.09 & 11.09 & 300 & 71.66 & 28.34 \\
\hline$-300+212$ & 15.24 & 15.24 & 212 & 56.42 & 43.58 \\
\hline$-212+150$ & 14.12 & 14.12 & 150 & 42.30 & 57.70 \\
\hline$-150+106$ & 10.40 & 10.40 & 106 & 31.90 & 68.10 \\
\hline$-106+75$ & 7.62 & 7.62 & 75 & 24.28 & 75.72 \\
\hline$-75+53$ & 5.96 & 5.96 & 53 & 18.32 & 81.68 \\
\hline$-53+38$ & 4.60 & 4.60 & 38 & 13.72 & 86.28 \\
\hline-38 & 13.72 & 13.72 & & & \\
\hline
\end{tabular}

Table III: Sieve analysis results for ilmenite ore

\begin{tabular}{|c|c|c|c|c|c|}
\hline \multirow{2}{*}{$\begin{array}{c}\text { Sieve size range } \\
(\mu \mathrm{m})\end{array}$} & \multicolumn{2}{|c|}{ Sieve fractions } & \multirow{2}{*}{$\begin{array}{l}\text { Nominal aperture size } \\
(\mu \mathrm{m})\end{array}$} & \multirow{2}{*}{ Cumulative \% undersize } & \multirow{2}{*}{ Cumulative \% oversize } \\
\hline & Weight (g) & Weight \% & & & \\
\hline+425 & 0.10 & 0.01 & 425 & 99.99 & 0.01 \\
\hline$-425+300$ & 0.10 & 0.01 & 300 & 99.98 & 0.02 \\
\hline$-300+250$ & 0.20 & 0.02 & 250 & 99.96 & 0.04 \\
\hline$-250+212$ & 0.50 & 0.05 & 212 & 99.91 & 0.09 \\
\hline$-212+150$ & 165.80 & 16.59 & 150 & 83.32 & 16.68 \\
\hline$-150+125$ & 661.30 & 66.16 & 125 & 17.17 & 82.83 \\
\hline$-125+106$ & 76.60 & 7.66 & 106 & 9.50 & 90.50 \\
\hline$-106+75$ & 88.00 & 8.80 & 75 & 0.70 & 99.30 \\
\hline$-75+53$ & 5.90 & 0.59 & 53 & 0.11 & 99.89 \\
\hline$-53+38$ & 1.00 & 0.10 & 38 & 0.01 & 99.99 \\
\hline-38 & 0.10 & 0.01 & & & \\
\hline
\end{tabular}

Table IV: Sieve analysis results for PGM ore from Platreef

\begin{tabular}{|c|c|c|c|c|c|}
\hline \multirow{2}{*}{$\begin{array}{c}\text { Sieve size range } \\
(\mu \mathrm{m})\end{array}$} & \multicolumn{2}{|c|}{ Sieve fractions } & \multirow{2}{*}{$\begin{array}{c}\text { Nominal aperture size } \\
(\mu \mathrm{m})\end{array}$} & \multirow{2}{*}{ Cumulative \% undersize } & \multirow{2}{*}{ Cumulative \% oversize } \\
\hline & Weight (g) & Weight \% & & & \\
\hline+1700 & 10.05 & 1.21 & 1700 & 98.79 & 1.21 \\
\hline$-1700+1180$ & 26.21 & 3.15 & 1180 & 95.65 & 4.35 \\
\hline$-1180+850$ & 105.00 & 12.61 & 850 & 83.04 & 16.96 \\
\hline$-850+500$ & 131.21 & 15.75 & 500 & 67.29 & 32.71 \\
\hline$-500+355$ & 92.97 & 11.16 & 355 & 56.12 & 43.88 \\
\hline$-355+212$ & 139.01 & 16.69 & 212 & 39.43 & 60.57 \\
\hline$-212+150$ & 117.80 & 14.14 & 150 & 25.29 & 74.71 \\
\hline$-150+106$ & 48.02 & 5.77 & 106 & 19.52 & 80.48 \\
\hline$-106+53$ & 95.27 & 11.44 & 53 & 8.09 & 91.91 \\
\hline-53 & 67.35 & 8.09 & & & \\
\hline
\end{tabular}


Table V: Sieve analysis results for PGM ore from Merensky reef

\begin{tabular}{|c|c|c|c|c|c|}
\hline \multirow{2}{*}{$\begin{array}{c}\text { Sieve size range } \\
(\mu \mathrm{m})\end{array}$} & \multicolumn{2}{|c|}{ Sieve fractions } & \multirow{2}{*}{$\begin{array}{c}\text { Nominal aperture size } \\
\text { (um) }\end{array}$} & \multirow{2}{*}{ Cumulative $\%$ undersize } & \multirow{2}{*}{ Cumulative \% oversize } \\
\hline & Weight (g) & Weight \% & & & \\
\hline+7100 & 3.02 & 0.74 & 7100 & 99.26 & 0.74 \\
\hline$-7100+5600$ & 7.46 & 1.84 & 5600 & 97.42 & 2.58 \\
\hline$-5600+4000$ & 33 & 8.12 & 4000 & 89.30 & 10.70 \\
\hline$-4000+1700$ & 74.50 & 18.34 & 1700 & 70.96 & 29.04 \\
\hline$-1700+1180$ & 41.20 & 10.14 & 1180 & 60.81 & 39.19 \\
\hline$-1800+600$ & 69.58 & 17.13 & 600 & 43.68 & 56.32 \\
\hline$-600+355$ & 44.52 & 10.96 & 355 & 32.72 & 67.28 \\
\hline$-355+150$ & 36.75 & 9.05 & 150 & 23.68 & 76.32 \\
\hline$-150+75$ & 22.88 & 5.63 & 75 & 18.04 & 81.96 \\
\hline$-75+45$ & 19.61 & 4.83 & 45 & 13.22 & 86.78 \\
\hline$-45+25$ & 18.87 & 4.65 & 25 & 8.57 & 91.43 \\
\hline-25 & 34.81 & 8.57 & & & \\
\hline
\end{tabular}

\section{B-5 Experimental data: Feed stream concentration}

\section{(a) Method employed for manual monitoring of feed stream concentration}

Since the hydrocyclone systems did not possess feed stream sampling points, the feed stream concentration was approximated by tallying the total mass of ore in the system. This approach is based on the assumption that the system concentration is similar to that of the feed stream. It is furthermore assumed that no mass is gained or lost from the system. As example, calculations for runs from the Gold case study are provided.

The amount of water introduced into the system is determined from the initial water level in the mixing tank and the mixing tank geometry. For the cylindrical mixing tank used during the Gold case study the total volume of water is

$$
\begin{aligned}
& \text { Volume }_{\mathrm{H}_{2} \mathrm{O}}=\pi \times\left(\text { Radius }_{\mathrm{Tank}}\right)^{2} \times \text { Height }_{\mathrm{H}_{2} \mathrm{O}} \\
& =\pi \times(0.375 \mathrm{~m})^{2} \times(0.333 \mathrm{~m}) \\
& =0.147 \mathrm{~m}^{3} .
\end{aligned}
$$

Using a water density of $1000 \mathrm{~kg} / \mathrm{m}^{3}$, the total mass of water in the system is calculated as

$$
\begin{aligned}
& \text { MasS }_{\mathrm{H}_{2} \mathrm{O}}=\text { Density }_{\mathrm{H}_{2} \mathrm{O}} \times \text { Volume }_{\mathrm{H}_{2} \mathrm{O}} \\
& =1000 \frac{\mathrm{kg}}{\mathrm{m}^{3}} \times 0.147 \mathrm{~m}^{3} \\
& =147 \mathrm{~kg} .
\end{aligned}
$$

During the first ore addition, $2.50 \mathrm{~kg}$ of gold ore was added to the system. The mass percent solids in the system (which serves as estimate for the feed stream concentration), is calculated as 


$$
\begin{aligned}
& \text { Mass } \% \text { solids }=\left(\frac{\text { Mass }_{\text {ore }}}{\text { Mass }_{\text {ore }}+\text { Mass }_{\mathrm{H}_{2} \mathrm{O}}}\right) \times 100 \% \\
& =\frac{2.50 \mathrm{~kg}}{2.50 \mathrm{~kg}+147 \mathrm{~kg}} \times 100 \% \\
& =1.7 \% .
\end{aligned}
$$

The tabulated data related to these examples of are highlighted in table VI.

\begin{tabular}{|c|c|c|c|c|}
\hline \multirow{2}{*}{ Run } & \multicolumn{2}{|c|}{ Ore addition } & \multirow{2}{*}{$\begin{array}{l}\text { Total mass in } \\
\text { system (kg) }\end{array}$} & \multirow{2}{*}{ Mass \% solids } \\
\hline & Per run (kg) & Cumulative (kg) & & \\
\hline 1 & 0.00 & 0.00 & 147.0 & 0.0 \\
\hline 2 & 2.50 & 2.50 & 149.5 & 1.7 \\
\hline 3 & 2.50 & 5.00 & 152.0 & 3.3 \\
\hline 4 & 7.50 & 12.50 & 159.5 & 7.8 \\
\hline 5 & 7.50 & 20.00 & 167.0 & 12.0 \\
\hline 6 & 7.50 & 27.50 & 174.5 & 15.8 \\
\hline 7 & 7.50 & 35.00 & 182.0 & 19.2 \\
\hline 8 & 7.50 & 42.50 & 189.5 & 22.4 \\
\hline 9 & 7.50 & 50.00 & 197.0 & 25.4 \\
\hline 10 & 2.50 & 52.50 & 199.5 & 26.3 \\
\hline 11 & 2.50 & 55.00 & 202.0 & 27.2 \\
\hline 12 & 2.50 & 57.50 & 204.5 & 28.1 \\
\hline 13 & 2.50 & 60.00 & 207.0 & 29.0 \\
\hline 14 & 2.50 & 62.50 & 209.5 & 29.8 \\
\hline 15 & 2.50 & 65.00 & 212.0 & 30.7 \\
\hline 16 & 2.50 & 67.50 & 214.5 & 31.5 \\
\hline 17 & 2.50 & 70.00 & 217.0 & 32.3 \\
\hline 18 & 2.50 & 72.50 & 219.5 & 33.0 \\
\hline 19 & 2.50 & 75.00 & 222.0 & 33.8 \\
\hline
\end{tabular}

\section{(b) Ore addition and concentration data}

Table VI: Concentration data for Gold case study

\begin{tabular}{|c|c|c|c|c|}
\hline \multirow{2}{*}{ Run } & \multicolumn{2}{|c|}{ Ore addition } & \multirow{2}{*}{$\begin{array}{l}\text { Total mass in } \\
\text { system (kg) }\end{array}$} & \multirow{2}{*}{ Mass \% solids } \\
\hline & Per run (kg) & Cumulative (kg) & & \\
\hline 20 & 2.50 & 77.50 & 224.5 & 34.5 \\
\hline 21 & 2.50 & 80.00 & 227.0 & 35.2 \\
\hline 22 & 5.00 & 85.00 & 232.0 & 36.6 \\
\hline 23 & 5.00 & 90.00 & 237.0 & 38.0 \\
\hline 24 & 5.00 & 95.00 & 242.0 & 39.3 \\
\hline 25 & 5.00 & 100.00 & 247.0 & 40.5 \\
\hline 26 & 5.00 & 105.00 & 252.0 & 41.7 \\
\hline
\end{tabular}

Table VI (continued): Concentration data for Gold case study 
Table VII: Concentration data for IImenite case study

\begin{tabular}{|c|c|c|c|c|}
\hline \multirow{2}{*}{ Run } & \multicolumn{2}{|c|}{ Ore addition } & Total mass in & Mass \% solids \\
\cline { 2 - 3 } & Per run (kg) & Cumulative (kg) & system (kg) & \\
\hline $\mathbf{1}$ & 0.00 & 0.00 & 212.0 & 0.0 \\
\hline $\mathbf{2}$ & 14.33 & 14.33 & 226.3 & 6.3 \\
\hline $\mathbf{3}$ & 10.31 & 24.64 & 236.6 & 10.4 \\
\hline $\mathbf{4}$ & 9.88 & 34.52 & 246.5 & 14.0 \\
\hline $\mathbf{5}$ & 5.45 & 39.97 & 252.0 & 15.9 \\
\hline $\mathbf{6}$ & 5.20 & 45.17 & 257.2 & 17.6 \\
\hline $\mathbf{7}$ & 6.17 & 51.34 & 263.3 & 19.5 \\
\hline $\mathbf{8}$ & 5.89 & 57.23 & 269.2 & 21.3 \\
\hline $\mathbf{9}$ & 6.01 & 63.24 & 275.2 & 23.0 \\
\hline $\mathbf{1 0}$ & 4.97 & 68.21 & 280.2 & 24.3 \\
\hline $\mathbf{1 1}$ & 5.55 & 73.76 & 285.8 & 25.8 \\
\hline $\mathbf{1 2}$ & 7.20 & 80.96 & 293.0 & 27.6 \\
\hline $\mathbf{1 3}$ & 9.19 & 90.15 & 302.2 & 29.8 \\
\hline $\mathbf{1 4}$ & 4.50 & 94.65 & 306.7 & 30.9 \\
\hline $\mathbf{1 5}$ & 4.79 & 99.44 & 311.4 & 31.9 \\
\hline $\mathbf{1 6}$ & 4.10 & 103.54 & 315.5 & 32.8 \\
\hline
\end{tabular}

Table VIII: Concentration data for Platreef case study

\begin{tabular}{|c|c|c|c|c|}
\hline \multirow{2}{*}{ Run } & \multicolumn{2}{|c|}{ Ore addition } & Total mass in & Mass \% solids \\
\cline { 2 - 3 } & Per run (kg) & Cumulative (kg) & system (kg) & \\
\hline $\mathbf{1}$ & 0.00 & 0.00 & 220.0 & 0.0 \\
\hline $\mathbf{2}$ & 5.00 & 5.00 & 225.0 & 2.2 \\
\hline $\mathbf{3}$ & 5.00 & 10.00 & 230.0 & 4.3 \\
\hline $\mathbf{4}$ & 2.50 & 12.50 & 232.5 & 5.4 \\
\hline $\mathbf{5}$ & 2.50 & 15.00 & 235.0 & 6.4 \\
\hline $\mathbf{6}$ & 2.50 & 17.50 & 237.5 & 7.4 \\
\hline $\mathbf{7}$ & 2.50 & 20.00 & 240.0 & 8.3 \\
\hline $\mathbf{8}$ & 2.50 & 22.50 & 242.5 & 9.3 \\
\hline $\mathbf{9}$ & 2.50 & 25.00 & 245.0 & 10.2 \\
\hline $\mathbf{1 0}$ & 2.50 & 27.50 & 247.5 & 11.1 \\
\hline
\end{tabular}


Table IX: Concentration data for Merensky 1 case study

\begin{tabular}{|c|c|c|c|c|}
\hline \multirow{2}{*}{ Run } & \multicolumn{2}{|c|}{ Ore addition } & Total mass in & Mass \% solids \\
\cline { 2 - 5 } & Per run (kg) & Cumulative (kg) & system (kg) & \\
\hline $\mathbf{1}$ & 0.00 & 0.00 & 251.3 & 0.0 \\
\hline $\mathbf{2}$ & 15.25 & 15.25 & 266.6 & 5.7 \\
\hline $\mathbf{3}$ & 15.70 & 30.95 & 282.3 & 11.0 \\
\hline $\mathbf{4}$ & 10.95 & 41.90 & 293.2 & 14.3 \\
\hline $\mathbf{5}$ & 10.90 & 52.80 & 304.1 & 17.4 \\
\hline $\mathbf{6}$ & 11.40 & 64.20 & 315.5 & 20.3 \\
\hline $\mathbf{7}$ & 10.00 & 74.20 & 325.5 & 22.8 \\
\hline $\mathbf{8}$ & 9.85 & 84.05 & 335.4 & 25.1 \\
\hline $\mathbf{9}$ & 6.10 & 90.15 & 341.5 & 26.4 \\
\hline $\mathbf{1 0}$ & 7.05 & 97.20 & 348.5 & 27.9 \\
\hline $\mathbf{1 1}$ & 7.00 & 104.20 & 355.5 & 29.3 \\
\hline $\mathbf{1 2}$ & 5.60 & 109.80 & 361.1 & 30.4 \\
\hline $\mathbf{1 3}$ & 7.20 & 117.00 & 368.3 & 31.8 \\
\hline $\mathbf{1 4}$ & 6.45 & 123.45 & 374.8 & 32.9 \\
\hline $\mathbf{1 5}$ & 8.65 & 132.10 & 383.4 & 34.5 \\
\hline
\end{tabular}

Table X: Concentration data for Merensky 2 case study

\begin{tabular}{|c|c|c|c|c|}
\hline \multirow{2}{*}{ Run } & \multicolumn{2}{|c|}{ Ore addition } & Total mass in & Mass \% solids \\
\cline { 2 - 5 } & Per run (kg) & Cumulative (kg) & system (kg) & \\
\hline $\mathbf{1}$ & 0.00 & 0.00 & 251.3 & 0.0 \\
\hline $\mathbf{2}$ & 41.20 & 41.20 & 292.5 & 14.1 \\
\hline $\mathbf{3}$ & 32.00 & 73.20 & 324.5 & 22.6 \\
\hline $\mathbf{4}$ & 13.45 & 86.65 & 338.0 & 25.6 \\
\hline $\mathbf{5}$ & 8.00 & 94.65 & 346.0 & 27.4 \\
\hline $\mathbf{6}$ & 19.10 & 113.75 & 365.1 & 31.2 \\
\hline $\mathbf{7}$ & 13.20 & 126.95 & 378.3 & 33.6 \\
\hline $\mathbf{8}$ & 20.70 & 147.65 & 399.0 & 37.0 \\
\hline $\mathbf{9}$ & 9.95 & 157.60 & 408.9 & 38.5 \\
\hline
\end{tabular}


Table XI: Concentration data for Merensky 3 case study

\begin{tabular}{|c|c|c|c|c|}
\hline \multirow{2}{*}{ Run } & \multicolumn{2}{|c|}{ Ore addition } & Total mass in & Mass \% solids \\
\cline { 2 - 5 } & Per run (kg) & Cumulative (kg) & system (kg) & \\
\hline $\mathbf{1}$ & 0.00 & 0.00 & 251.3 & 0.0 \\
\hline $\mathbf{2}$ & 29.45 & 29.45 & 280.8 & 10.5 \\
\hline $\mathbf{3}$ & 19.05 & 48.50 & 299.8 & 16.2 \\
\hline $\mathbf{4}$ & 14.80 & 63.30 & 314.6 & 20.1 \\
\hline $\mathbf{5}$ & 13.20 & 76.50 & 327.8 & 23.3 \\
\hline $\mathbf{6}$ & 12.55 & 89.05 & 340.4 & 26.2 \\
\hline $\mathbf{7}$ & 9.00 & 98.05 & 349.4 & 28.1 \\
\hline $\mathbf{8}$ & 6.50 & 104.55 & 355.9 & 29.4 \\
\hline $\mathbf{9}$ & 6.55 & 111.10 & 362.4 & 30.7 \\
\hline $\mathbf{1 0}$ & 9.00 & 120.10 & 371.4 & 32.3 \\
\hline $\mathbf{1 1}$ & 10.80 & 130.90 & 382.2 & 34.2 \\
\hline $\mathbf{1 2}$ & 17.35 & 148.25 & 399.6 & 37.1 \\
\hline
\end{tabular}




\section{APPENDIX C: \\ IMAGE ANALYSIS AND DIAGNOSTIC MONITORING DE'TAILS}

\section{C-1 Sample calculations: Image enhancement}

For the Merensky 1 case study, the following inputs were specified for the intensity range transformation:

- Minimum of intensity range $=93$

- Maximum of intensity range $=159$

- Centre of intensity range $=123$

To implement the desired contrast adjustment, the specified minimum and maximum values of the intensity range were used to obtain the equation of the sloped section of the transformation function (as depicted in figure II):

Output $=m \times$ Input $+c$

Equation I

where

Output $=$ output intensity value,

$m=$ slope,

Input $=$ input intensity value and

$c=$ intercept.

The slope was consequently calculated as,

$m=255 /($ Maximum - Minimum $)$

Equation II

$=\frac{255}{159-93}$

$=3.86$

and the intercept as

$c=-m \times$ Minimum

Equation III

$=3.86 \times 93$

$=-355$. 


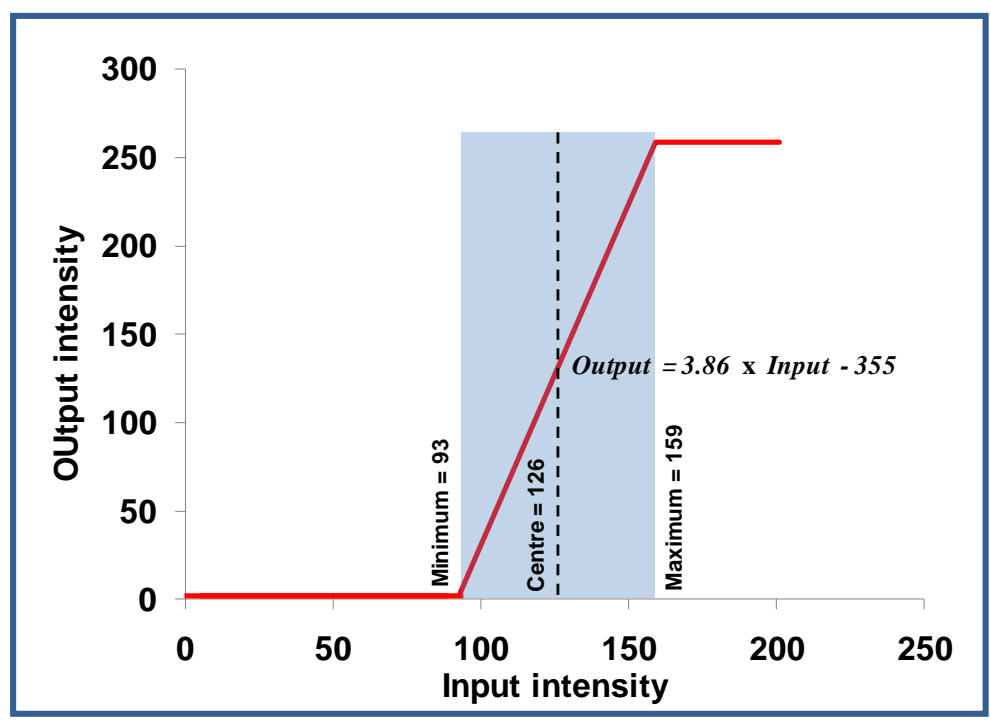

Figure II: Transformation function for contrast adjustment of Merensky 1 case study

Concerning the brightness adjustment, the default centre of the specified range, 126, was reset to the specified centre 123 . Consequently, the whole transformation function was moved 3 intensity values to the left, as illustrated in figure III.

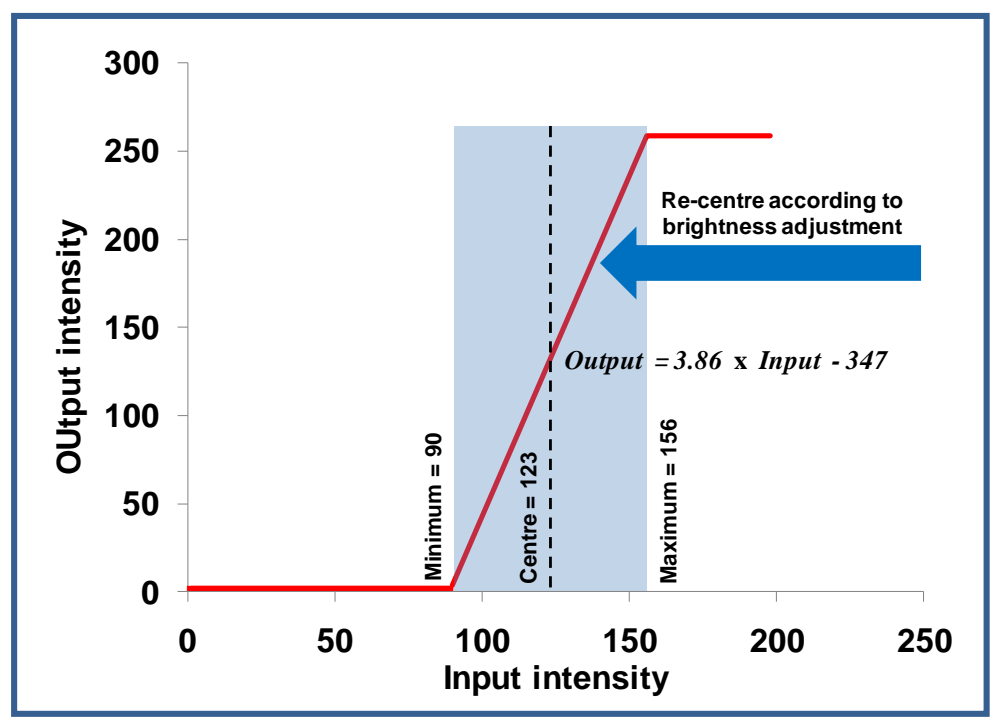

Figure III: Transformation function for contrast and brightness adjustment of Merensky 1 case study 


\section{C-2 Specified user inputs for different case studies}

Table XI: Case study sizes and specified user inputs

\begin{tabular}{|c|c|c|c|c|c|c|c|}
\hline \multirow{2}{*}{ Case study } & \multirow{2}{*}{$\begin{array}{c}\text { Size } \\
\text { (number of frames) }\end{array}$} & \multicolumn{3}{|c|}{$\begin{array}{l}\text { Contrast and brightness } \\
\text { adjustments }\end{array}$} & \multirow{2}{*}{$\begin{array}{l}\text { Horizontal line } \\
\text { position } \\
\text { (y-coordinate) }\end{array}$} & \multirow{2}{*}{$\begin{array}{c}\text { Motion } \\
\text { threshold* }\end{array}$} & \multirow{2}{*}{$\begin{array}{l}\text { Training } \\
\text { data }\end{array}$} \\
\hline & & Minimum & Maximum & Centre & & & \\
\hline Gold & 190964 & 87 & 162 & $\begin{array}{c}125 \\
\text { (Default) }\end{array}$ & 240 & 18 & $\begin{array}{c}58405 \\
\text { to } \\
173099\end{array}$ \\
\hline Platreef & 25768 & 90 & 162 & $\begin{array}{c}126 \\
\text { (Default) }\end{array}$ & 10 & 18 & $\begin{array}{c}0 \\
\text { to } \\
23430\end{array}$ \\
\hline Merensky 1 & 69463 & 93 & 159 & 123 & 190 & 18 & $\begin{array}{c}31020 \\
\text { to } \\
68490\end{array}$ \\
\hline Merensky 2 & 42277 & 87 & 157 & 125 & 220 & 18 & $\begin{array}{c}10230 \\
\text { to } \\
42060 \\
\end{array}$ \\
\hline Merensky 3 & 84836 & 87 & 157 & 125 & 220 & 13 & $\mathrm{~N} / \mathrm{A}$ \\
\hline
\end{tabular}

\section{C-3 Multivariate Gaussian distribution}

The MVGD probability density function is defined by equation IV.

$f(u)=\frac{1}{\sqrt{\left|\sum\right|(2 \pi)^{r}}} e^{-\frac{1}{2}(u-\mu) \sum^{-1}(u-\mu)^{\prime}}$

where

$u=$ random variable (in this case embedded underflow width vector),

$\mu=$ mean,

$\Sigma=$ variance and

$r=$ number of variables.

\footnotetext{
*Value of motion threshold specifies minimum intensity difference detected as motion
} 


\section{APPENDIX D: ADDITIONAL RESULTS}

\section{D-1 Underflow width versus feed stream concentration}

(a)

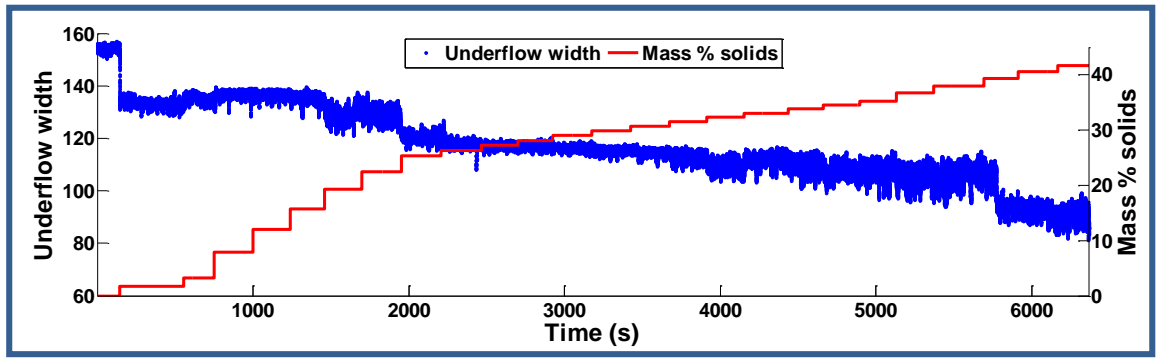

(b)

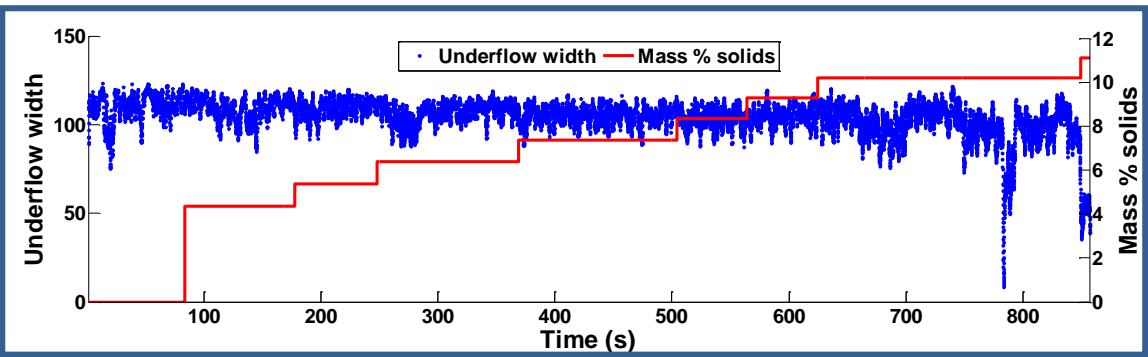

(c)

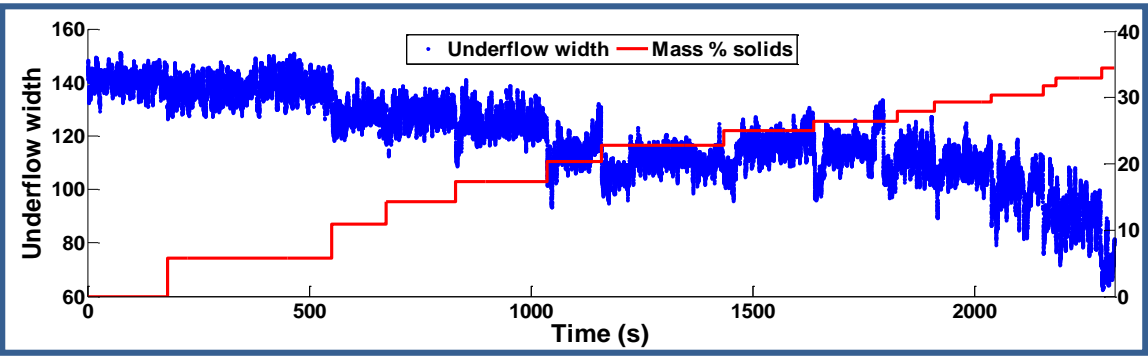

(d)

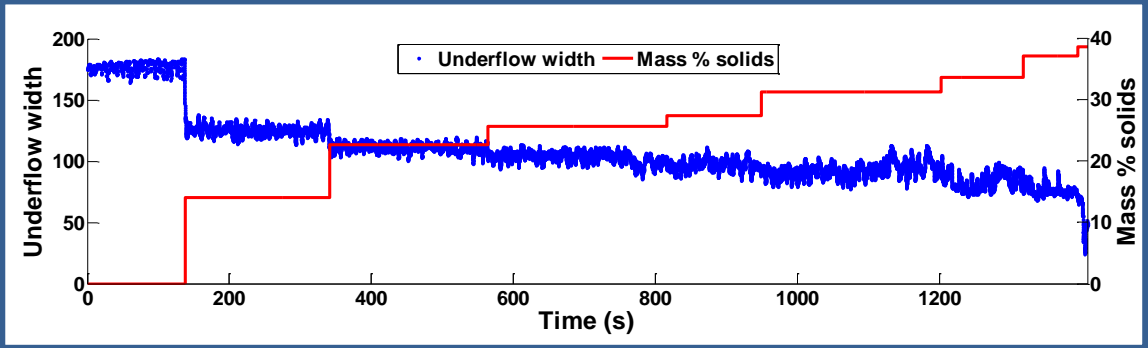

(e)

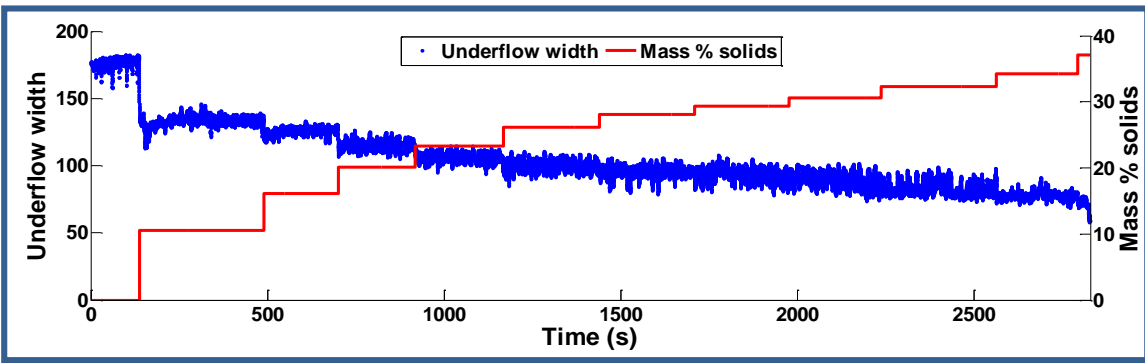

Figure IV: Underflow width against feed stream concentration for (a) Gold, (b) Platreef, (c) Merensky 1, (d) Merensky 2 and (e) Merensky 3 case studies 


\section{D-2 Automatically selected monitoring parameters}

Table XII: Automatically selected monitoring parameters

\begin{tabular}{|c|c|c|c|}
\hline Case study & $\begin{array}{c}\text { Embedding } \\
\text { lag, } \tau\end{array}$ & $\begin{array}{c}\text { Corresponding } \\
\text { sampling time } \\
\text { (s) }\end{array}$ & $\begin{array}{c}\text { Kernel } \\
\text { parameter, } \gamma\end{array}$ \\
\hline Gold & 132 & 5.4 & $2^{-5}$ \\
\hline Platreef & 29 & 2.0 & $2^{-7}$ \\
\hline Merensky 1 & 50 & 2.7 & $2^{-7}$ \\
\hline Merensky 2 & 81 & 3.7 & $2^{-7}$ \\
\hline Merensky 3 & 81 & 3.7 & $2^{-7}$ \\
\hline
\end{tabular}

The sampling time corresponding to an embedding lag was calculated by taking the moving average and shutter speed into account. For example, for the Gold case study the corresponding sampling time was calculated as:

(Points required to calculate $1^{\text {st }}$ moving average + Embedding lag)/Shutter speed

$=(30+132) / 30$

Equation V

$=5.4 \mathrm{~s}$ 